Check for updates

Cite this: Mater. Adv., 2021, 2, 186

Received 20th June 2020, Accepted 2nd October 2020

DOI: $10.1039 / \mathrm{d} 0 \mathrm{ma} 00439 \mathrm{a}$

rsc.li/materials-advances

\section{LaMer's 1950 model of particle formation: a review and critical analysis of its classical nucleation and fluctuation theory basis, of competing models and mechanisms for phase- changes and particle formation, and then of its application to silver halide, semiconductor, metal, and metal-oxide nanoparticles $\dagger$}

\author{
Christopher B. Whitehead, (D) ${ }^{a}$ Saim Özkar (D) ${ }^{b}$ and Richard G. Finke (D) *a
}

\begin{abstract}
A review is presented of the pioneering 1950 model (V. K. LaMer, R. H. Dinegar, Theory, Production and Mechanism of Formation of Monodispersed Hydrosols, J. Am. Chem. SoC., 1950, 72, 4847-4854) of how monodisperse particles might possibly be formed. The review begins with a look at the basis of the 1950 model in fluctuation and classical nucleation theories. Presented next are the competing phasechange models and then also chemical mechanisms for particle formation available since the 1950 paper, including a little-cited nucleation mechanism that LaMer insightfully wrote in 1952. This review then takes a critical look at the $164(\sim 8 \%)$ of the 192 total ( 10\%) out of the 1953 papers (as of March 2019) that cite the 1950 model while also providing at least some discussion, analysis, or additional data bearing on the 1950 model postulating "effectively infinite nucleation" and "diffusion-controlled growth". (The other 28 papers out of the 192 total papers describe $S_{n}$ sol formation were covered in an earlier, Part I review that is cited.) Those 164 papers are broken down into five tables provided in the Supporting Information and are then covered in separate sections in the main text: first 13 papers on silver halide nanoparticles (Table S1) where the single best evidence in support of the 1950 model has been thought to exist; 26 papers on semiconductor nanoparticles (Table S2); then 69 papers on transition-metal nanoparticle formation (Table S3); 39 papers on oxide-based nanoparticles (Table S4); and 17 papers presenting alternative models or mechanisms in comparison to the 1950 model (Table S5). The review focuses on answering the critical question of: do the concepts of "burst/ instantaneous nucleation" and "diffusion-controlled growth" have sound, compelling experimental support in the 70 years since the model first appeared and in the 164 papers examined more closely that do more than just cite the 1950 model? A Conclusions section listing sixteen bullet points is provided, as is a final section entitled "A Look Towards the Future" that discusses evolving areas and suggested emphasis points for facilitating future research in particle formation kinetics, mechanism and associated particle syntheses.
\end{abstract}

\footnotetext{
${ }^{a}$ Department of Chemistry, Colorado State University, Fort Collins, CO 80523, USA. E-mail: Richard.Finke@colostate.edu

${ }^{b}$ Department of Chemistry, Middle East Technical University, 06800 Ankara, Turkey $\dagger$ Electronic supplementary information (ESI) available: Fig. S1-S7 and Table S1, summary of literature on the formation of silver halide nanoparticles relating to the 1950 model; Table S2, summary of literature on the formation of semiconductor nanoparticles relating to the 1950 model; Table S3, summary of transitionmetal nanoparticles literature relating to the 1950 model; Table S4, summary of the literature on oxide-based nanoparticles relating to the 1950 model; Table S5, summary of other, alternative models or mechanisms for nanoparticle formation that cite or impact the 1950 model. See DOI: 10.1039/d0ma00439a
}

\section{Introduction}

Seventy years ago, LaMer and co-workers postulated a model ${ }^{1}$ for particle formation consisting of nucleation with an effectively infinite rate and then diffusion-controlled growth, a classic paper that has yielded over 1953 citations as of the time this review was constructed. As described in Fig. 1 on page 4847 of the 1950 paper, "The rate of nucleation, as judged by the reproducibility of the timing and the concentration of sulfur at which the system becomes heterogeneous, is so exceedingly 
sensitive to an increase in the concentration of dissolved sulfur that the rate becomes effectively infinite". ${ }^{1}$ Hence the words used since then in many of the aforementioned total citations of the 1950 model of nucleation as "instantaneous" or "burst" are an accurate citation of the model in at least its most widely cited, schematic/qualitative form that is Fig. 1 of the classic 1950 paper. $^{1}$ The significance of "instantaneous or burst"

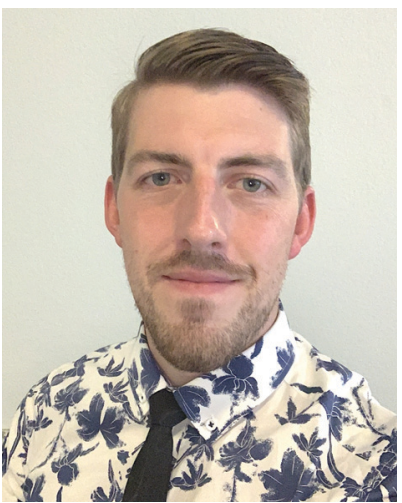

Christopher B. Whitehead
Christopher B. Whitehead is a graduate student at Colorado State University. He received his $B A$ in chemistry from Willamette University in 2014 and will complete his PhD in chemistry with Prof. Richard Finke at Colorado State University in 2020 while focusing on mechanistic studies of nanoparticle formation. During his doctoral studies he authored/coauthored 10 peer-reviewed research papers. His current research interests center around the intricacies of the nucleation step, improvements to semiconductor/quantum dot materials, and applications to energy collection and storage.

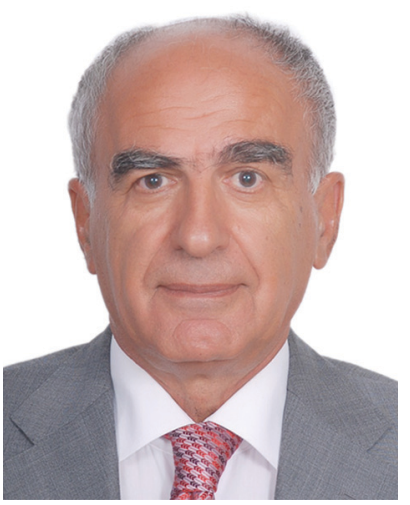

Saim Özkar
Saim Özkar is a Professor of Chemistry at the Middle East Technical University (METU) in Ankara, Turkey. He graduated from Istanbul Technical University in 1972, received his PhD at the Technical University of Munich in 1976, joined METU in 1979, and became a Full Professor in 1988. He spent one year at Max Planck Institute in Mülheim in 1986, 2 years at University of Toronto in 1988, and now 5 total years at Colorado State University since 2000 with the Finke research group resulting in 32 papers from that collaboration and his now 20 years of experience in metal-nanoparticle chemistry. He was awarded 1996 Science Prize by Scientific and Technological Research Council of Turkey and has been member of Turkish Academy of Sciences since 1996. nucleation and continuing allure of the 1950 model seems clear: it at least previously (vide infra) was the only way to explain the formation of (near) "monodisperse" particles, the word monodisperse appearing in the title of the famous 1950 paper, "Theory, Production and Mechanism of Formation of Monodispersed Hydrosols". ${ }^{1}$

\subsection{The Part I review}

Recently, in our Part I review ${ }^{2}$ we provided 13 sections in a first attempt to begin to look critically and comprehensively at the extensive literature citing the 1950 model. More specifically, in our Part I review we started the effort needed to address the important question of what experimental evidence for that 1950 model has accumulated in 70 years and via 1953 papers citing the classic 1950 paper. ${ }^{1}$ Those 13 sections in our Part I review are: (i) the chemical origins of the 1950 model; (ii) the original sulfur hydrosol formation system; (iii) the often-cited schematic/qualitative, and then associated words-only descriptions of the 1950 model; (iv) the nine assumptions underlying the little employed quantitative mathematical model and associated equation in the 1950 paper; (v) the question of "Is LaMer's model actually a 'growth model'"?; (vi) the actual quantitative equation in the 1950 paper-plus the question of when has it been used in the last 70 years to fit particle formation kinetics data?; (vii) the disconnect between the 1950 quantitative model and associated differential equation

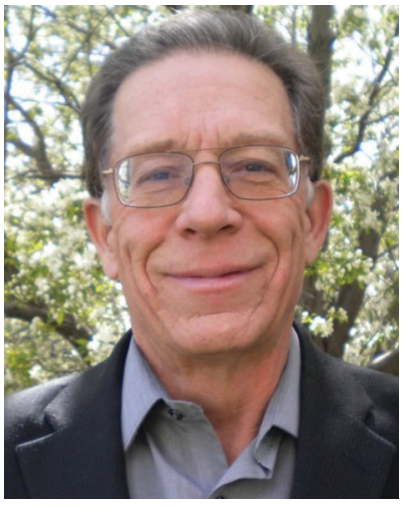

Richard G. Finke
Richard G. Finke is a Professor of Chemistry at Colorado State University. He received his $B A$ with Distinction from the University of Colorado, and his PhD in Chemistry from Stanford University in 1976 under Professors James P. Collman and John I. Brauman. He was an NSF postdoctoral fellow in 1977, also at Stanford University. His awards include A. P. Sloan, Dreyfus Teacher-Scholar, and Guggenheim Fellowships, a Colorado ACS Section Award, Colorado State University Scholarship Impact Award, and Excellence in Graduate Education and Mentoring Award. His recent research activities focus on kinetics and mechanistic studies of nanoparticle formation involving nucleation, growth, and agglomeration, answering the related question of "what is the true catalyst?" in reactions that could be either homogeneous or heterogeneous catalysis, and other areas of chemical catalysis including artificial photosynthesis for fuel-forming reactions plus water-oxidation catalysis involving polyoxometalates or metal-oxide nanoparticles. In the past he has contributed to mechanistic bio-inorganic chemistry of coenzyme $B_{12}$ and to mechanistic organo-lanthanide, organoactinide, and electro-chemistries. 
$v s$. any testing against experimental kinetics data; (viii) a look at the kinetics data for, and the current understanding of, the mechanism of sulfur sol formation; (ix) the 1926, first kinetics data for sulfur sol formation; (x) light scattering data for sulfur sol formation on which the 1950 model was originally founded; (xi) fitting of the 1926 kinetics data for sulfur sol formation by a minimalistic 2-step mechanism; ${ }^{3}$ (xii) the interesting effect of microfiltration and the removal of dust on the size distribution of sulfur sols; and then (xiii) a summary and conclusions section. ${ }^{2}$ Reading the Part I review prior to reading the present Part II review is recommended in order to fully understand what follows. That said, every attempt has been made to make the following, Part II review as self-contained as possible.

\subsection{The contents of the present, Part II review}

What was not done in the Part I review, ${ }^{2}$ and hence is a focus of the present Part II review of the 1950 model, is to critically examine the remaining $\sim 8 \%$ of the 1953 citations (164 papers) that do more than merely cite the 1950 model (i.e., and which go beyond the 28 papers on $S_{n}$ sol formation that gave rise to the 1950 model and are covered in our Part I review ${ }^{2}$ ). Any associated, relevant literature needed to understand and place in context those 164 papers is also presented. In particular, the present Part II review also looks at key theories that underlie the 1950 mathematical model and that were not presented in the Part I review, notably classical nucleation theory (CNT) and the related topic of fluctuation theory. Also examined are the other models and chemical mechanisms since 1950 available for interpreting particle formation and other phase-change kinetics and related data.

Specifically then and to start, the relevant background materials are presented of (i) CNT and fluctuation theory, vide infra, (ii) LaMer's 1952 paper $^{4}$ on nucleation in phase transitions, including his little-cited experimental investigations of $\mathrm{BaSO}_{4}$ formation, and (iii) Turkevich's groundbreaking 1951 and 1953 papers $^{5,6}$ on the synthesis of gold colloids. The overarching goal of this Part II review is to collect and critically examine the experimental evidence gathered in the last 70 years that provides support for (or against) "instantaneous/ burst" nucleation followed by "diffusion controlled" growth.

Our own interest in the experimental evidence for the 1950 model began in $1994^{7}$ when we observed the reproducible formation of near-monodisperse ( $\leq 15 \%$ polydispersity) $\operatorname{Ir}(0)_{n}$ nanoparticle formation. ${ }^{7}$ Those particles were remarkable at the time in that they disproved several myths: that one could not have a "colloid" outside of water solvent; that one could not have reproducible formation of a "colloidal"; and that no "colloid" could be isolated and bottled (i.e., that all "colloids" had a "critical colloid concentration" past which they could not be concentrated without aggregation). ${ }^{7}$ But even more intriguing to us was how could any such particle having anything like $n=300$ to $900 \operatorname{Ir}(0)$ atoms as in our $\operatorname{Ir}(0)_{n}$ systems, and therefore mechanisms that had to have at least 300-900 steps (and likely several 1000s of steps), ever possible yield reproducible, near-monodisperse, particles? What is the mechanism(s) for such a complex, multistep particle formation process?
A search of the literature at the time (pre-1994) ${ }^{7}$ led to the 1950 model $^{1}$ and its thermodynamically based, burstnucleation and diffusion-controlled growth hypothesis for particle formation. Yet there were hints in the literature even then that the "burst nucleation" model might not be generally applicable, or perhaps not even correct. E. Matijevic noted in his 1993 review describing his pioneering work on the "Preparation and Properties of Uniform Size Colloids" that “...the recognition of the limitations of the 1950 model came through a number of experimental findings that contradicted it." ${ }^{, 8}$ In 1994 we noted for our $\operatorname{Ir}(0)_{n}$ nanoparticle formation system that the nucleation and growth steps were “...much smoother and more continuous than the popular and often cited 'burst' nucleation phenomenon from a supersaturated solution associated with LaMer's mechanism."7 Our 1994 paper $^{7}$ contained a preliminary version of the disproof-based, deliberately minimalistic, pseudo-elementary 2-step mechanism for particle formation that we published in full form in 1997 composed of kinetically "continuous nucleation" plus "autocatalytic surface growth". ${ }^{3}$ In that work we noted, at the time and in our opinion, that "LaMer's pioneering work" may have been "inappropriately cited", a comment intended to note that any such incorrect citations were not 1950s author's fault but, instead, "a phenomenon which really only points to the dearth of new, broadly applicable and kinetically verified alternative mechanisms in the intervening nearly 50 years."3

In the ensuing 23 years we continued to read the many papers citing the creative and pioneering 1950 work. ${ }^{1}$ The more we read, the more we became increasingly puzzled by continued, wide citation of the 1950 model despite the lack of experimental evidence, that at least we could find, in support of a particle formation model involving true instantaneous/ burst nucleation followed by diffusion-controlled growth. Relevant here is this word version of the 1950 model often being cited as if it were compellingly documented, disproof-based, scientific fact. Several years back we decided it was finally time to put in the effort required to examine critically the 1953 available references we found (as of March 2019) that cited the classic 1950 paper. That exhaustive literature search revealed that it was especially important to focus on 164 papers we identified that did more than just cite the 1950 model in passing.

Hence, the present Part II review is the long-overdue, needed review and analysis of those 164 papers accomplished via an extensive (74 pages of) ESI, $\dagger 5$ tables with individual assessments and summaries for each paper, and then the main text and discussion which follows. The attached 5 tables and ESI $\dagger$ are intended as an introductory resource that readers can refer to and use as desired. Moreover, the reader that wants to be driven to their own, independent conclusions about the 1950 model and where particle formation mechanisms and science stand can review those five tables carefully-indeed, we urge this self-study of the ESI $\dagger$ several times in the presentation and discussion that follows.

Our goal of this Part II review is not to support any specific model or mechanism, nor any of those listed in Tables 1 and 2 for instance, vide infra, especially since several of the 
mechanistic models are from our own work. Instead, our goal via our Part I and Part II reviews is to provide the community with the first comprehensive look at the burst nucleation and diffusion-controlled growth model with an emphasis on the experimental evidence testing it over the intervening 70 year period. We start by a short review of classical nucleation theory that underlies the 1950 model, and the related topic of fluctuation theory.

\subsection{Classical nucleation theory: a concise look at an often misapplied theory that underlies the 1950 model}

Classical nucleation theory (CNT) was developed in an attempt to explain the phenomenon of nucleation, ${ }^{9-13}$ a theory that continues to be the most popular model for nucleation even today. ${ }^{14,15}$ The widespread application of CNT continues across many types of systems in nature despite the now well-demonstrated inapplicability of CNT to strong-bonding (also called "strongly associating") systems ${ }^{16,17}$ and to cases involving high levels of supersaturation where CNT was never claimed to work, vide infra. It is now abundantly clear that CNT is misapplied if used beyond weakly bonding ("weakly associating") systems, an example of a weakly bonding system being hydrocarbon ( $\mathrm{RH}$ ) aggregation in the gas phase where CNT has some of its best success as discussed more in a moment. Relevant here is that applying classic theories far beyond their original, intended applications and beyond the limits of their assumptions is a documented, more general problem in chemical science as described in a highly recommended paper by B. Peters. ${ }^{18}$ Additionally, the dominant effect of surface ligands on nanoparticles that will be apparent throughout the review, plus the fact that CNT lacks any provision for handling ligands (is "ligand-blind"), means that CNT cannot possibly handle nucleation in most modern systems that include binding ligands, except conceivably by accident.

The mathematical foundation for CNT was developed in the late 19th century by Gibbs, ${ }^{19}$ with the now approaching 100 years-old theory being constructed in the early 20 th century by Farkas, ${ }^{20}$ Volmer, ${ }^{21,22}$ Becker and Döring, ${ }^{23}$ Frenkel, ${ }^{24}$ and Zeldovich. ${ }^{25}$ Critical to understanding CNT and where it should or should not be applied is to realize that CNT is a thermodynamic-based theory-a hypothesis-for nucleation that we now know is more generally a kinetically controlled process. $^{2,3,5,6,10-14,16,17,26,27}$ In the thermodynamically based CNT, the free energy change of the particle formation is defined/assumed to be the sum of the free energy changes of the phase transformation and formation of the surface, Fig. 1. Additional assumptions employed in CNT that are most relevant to this review include, but are not limited to: ${ }^{16}$ (i) the so-called capillary approximation, whereby the molecular arrangement of the droplet (a, huge, oversized "nucleus", from this macroscopic, top-down approach) is assumed to be identical to that of the bulk, macroscopic material, ${ }^{2}$ a crude approximation/assumption that is perhaps never true; (ii) that the surface energy is independent of temperature; and (iii) that surface growth is assumed to occur one monomer at a time with collisions between two or more particles being neglected

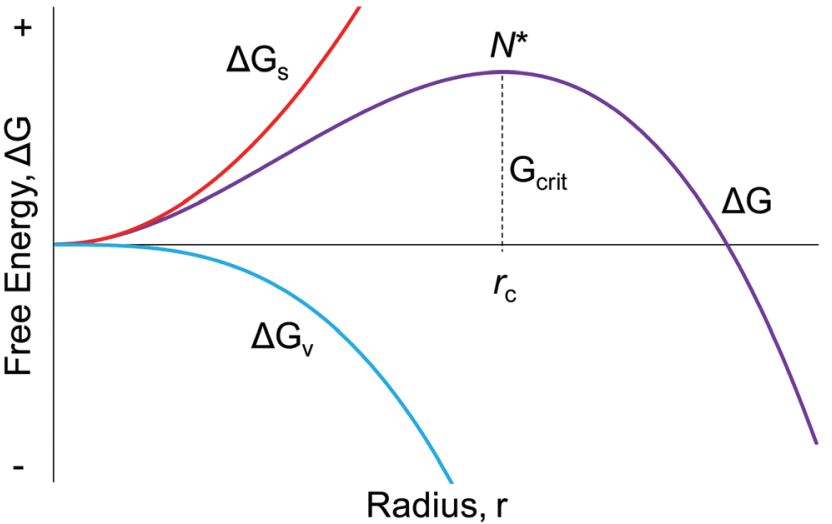

Fig. 1 The classic, widely cited, free energy diagram of $\mathrm{CNT}^{9}$ in which $\Delta \mathrm{G}$ for the particle formation is plotted against the radius, $r$, of the assumed spherical particle. Important variables in CNT are: $\Delta G_{\mathrm{s}}$, the free energy of the particle's surface; $\Delta G_{v}$, the free energy of the bulk crystal; $r_{\mathrm{c}}$, the critical radius which is the minimum size required for the particle to form without being re-dissolved; and $G_{\text {crit, }}$ the maximum free energy of the particle that is the definition of the "critical radius", $N^{*}$

(i.e., agglomeration not being allowed). Hence, (iv) the assumed underlying mechanism for particle formation is reversible monomer addition, ${ }^{16,28} \mathrm{~A}+\mathrm{A} \rightleftharpoons \mathrm{A}_{2}, \mathrm{~A}_{2}+\mathrm{A} \rightleftharpoons \mathrm{A}_{3}, \ldots \mathrm{A}_{n-1}+$ $\mathrm{A} \rightleftharpoons \mathrm{A}_{n}$ as detailed in Reiss' highly recommended review of $\mathrm{CNT}^{28}$ Additionally, CNT assumes (v) that "the stationary distribution of subcritical solute cluster distribution is established instantaneously (italics added) after the onset of supersaturation"16 (see also Fig. 2 in Reiss' 1986 paper for a schematic showing a proposed effectively infinite nucleation rate $\left.^{28}\right)$. That is, CNT posits instantaneous/burst nucleation, even though it is the time dependence and kinetics of nucleation that one needs to measure along with other important factors such as the composition and structure of the nuclei and the number of nuclei $v s$. time. In CNT (vi) the nucleation rate is actually assumed to be independent of time, which implies a chemically and mechanistically implausible, zero-order process-rather than the ostensibly $n$th order implied by the reversible associations shown for the postulated overall $n \mathrm{~A} \rightleftharpoons \mathrm{A}_{n}$ in (iv) above.

The CNT expression used to describe the free energy of the particle in terms of the particle radius is then given as eqn (1) with its competing, negative and positive terms: ${ }^{29}$

$$
\Delta G=-\frac{4}{3} \pi r^{3}\left|\Delta G_{\mathrm{v}}\right|+4 \pi r^{2} \gamma
$$

Eqn (1) assumes a spherical nucleus because a sphere minimizes the surface area of the nucleus. Here, $r$ is the radius of the particle, $\Delta G_{\mathrm{v}}$ is the difference of free energy per volume between the old bulk phase and the new bulk phase, and $\gamma$ is the free energy of the surface per unit area.

An often-overlooked aspect of the putative "critical nucleus" of CNT as defined in Fig. 1 is that the critical nucleus is a theoretical concept, not something one expects to be able to observe experimentally. The "critical nucleus" as described by CNT is the most unstable transient at the top of the combined 
$\Delta G$ curve according to CNT. As noted by Professor H. Reiss already back in 1986 (on p. 127 of his paper ${ }^{28}$ ) "the nuclei themselves are never observed; only the entities which develop from them can be interrogated". ${ }^{28}$ Restated, papers professing to have observed the "Critical Nucleus" of CNT are, in all probability, actually seeing what has been called, instead and in modern particle-formation language, the first observable cluster $(\text { FOC })^{17}$ - that is, the FOC detected by the inherent, limiting sensitivity of whatever physical method is being employed.

What is experimentally measurable is the kinetics of nucleation, including the reaction order, $n$, in the precursor concentration (i.e., $[\mathrm{A}]^{n}$ ) of the nucleation step, ${ }^{3,17,26,27}$ that reaction order nominally giving one the nuclearity $\left(\mathrm{A}_{n}\right)$ of the what has recently been defined as the kinetically effective nucleus (KEN). ${ }^{17}$ Such an experimental measurement of the nucleation reaction order also looks to be one version of the so-called "Nucleation Theorem"; see p. 1963, 1970 and ref. 132, 133 and 139 provided in ref. 12 . Of considerable interest and importance here is the mounting evidence that, in at least stronglybonded systems, the KEN may more generally be of low nuclearity, perhaps often just 2-3 (i.e., $\mathrm{A}_{2-3}$ ), and hence much smaller experimentally than the critical nucleus, $\mathrm{A}_{n}$, of CNT where $n \gg 2-3$ has at least historically been implied.

Hence, the pen-and-paper theory-really the hypothesis; the mathematical model-we know as CNT is what was possible at the time. ${ }^{19-25}$ It is based on the thermodynamic approach possible at the time. An approach to the kinetics of nucleation would have had to account for the transition state, a challenging job even today with all of our modern computational prowess and powerful physical methods. CNT works best for vapor phase, single component, weakly associating systems at relatively low levels of supersaturation such as the gas-phase aggregation of hydrocarbons ${ }^{30,31}$ - that, therefore, can have the facile $n \mathrm{~A} \rightleftharpoons \mathrm{A}_{n}$ assembly, disassembly kinetics required by the thermodynamic basis of CNT. CNT therefore looks to work best for "weakly-bonded" or "weakly associating" systems that are also not far beyond supersaturation. CNT was never claimed to be valid at large supersaturations ${ }^{19-25}$ such as one can easily run into for strong-bonded, low solubility systems such as zerovalent metals as one example. Hence, CNT was never intended for-and therefore should not be applied to- "strong-bonded" nucleating systems such as transition-metal nanoclusters to pick a specific example.

For the case of $\operatorname{Ir}(0)_{n}$ nanoparticles as just one example, each new Ir-Ir bond is worth an estimated $\sim 26 \mathrm{kcal} \mathrm{mol}^{-13,17}$ and " $\operatorname{Ir}(0)$ " metal is generally listed as "insoluble",32 so that one cannot speak of its level of "supersaturation". Hence, the reversible aggregation of monomers, $n \mathrm{~A} \rightleftharpoons \mathrm{A}_{n}$, for such a relatively strong bonding system is not plausible thermodynamically because every $\mathbf{M}-\mathbf{M}$ bond made is worth $\sim 26 \mathrm{kcal} \mathrm{mol}^{-1}$ corresponding to an associated $\Delta H_{\text {vaporization }}$ of $159 \mathrm{kcal} \mathrm{mol}^{-1}$ in at least (bulk) $\operatorname{Ir}(0)_{n} \cdot{ }^{3,17}$ The presence of excess ligands, $\mathrm{L}$, and when the $\mathrm{M}-\mathrm{L} v s$. $\mathrm{M}-\mathrm{M}$ bond energies are comparable, could change the situation and could lead to thermodynamic reversibility in some of the nucleation step(s), at least in principle.
However, more to the point, $\operatorname{Ir}(0)$ is not involved in the nucleation step in the example of a $\{(1,5-\mathrm{COD}) \mathrm{Ir} \cdot \mathrm{POM}\}^{8-}$ precursor $(=\mathrm{A})$ reduced under $\mathrm{H}_{2}$ to yield isolable nearmonodisperse nanoparticles, $\left\{\operatorname{Ir}(0)_{n} \cdot(\mathrm{POM})_{x}\right\}^{-9 x}$ (where $\mathrm{POM}^{9-}$ is a custom-made, polyoxometalate stabilizing ligand). There the kinetically effective nucleus composition has been determined via classical kinetics dependence on $[\mathrm{A}]$ and other experiments to be $\left\{\operatorname{Ir}_{3}^{\mathrm{I}} \mathrm{H}_{2 x} \cdot \mathrm{POM}\right\}^{6-} \cdot{ }^{26}$ Hence, the stronger metal-hydride, $\mathrm{M}-\mathrm{H}-\mathrm{M}$, bond is involved, ${ }^{17,26,27}$ for reference a single $\mathrm{Ir}-\mathrm{H}$ bond energy is on the order of $\sim 75 \mathrm{kcal} \mathrm{mol}^{-1} \cdot{ }^{17}$ In this system the evidence is strong that nucleation does not involve a reversible, $n \mathrm{~A} \rightleftharpoons \mathrm{A}_{n}$ system but, instead, involves a low nuclearity ${ }^{17,26,27}$ KEN with a $n=3 .^{26}$ This is a good example of where classical experimental kinetics, but now of nucleation, are both necessary and powerful, kinetics generally giving one the composition of the transition state of the rate-determining step in all but a few special cases.

Additional evidence for the inapplicability of CNT to $\mathbf{M}(0)_{n}$ systems comes from studies of nucleation to form $\operatorname{Li}(0)_{n}$ or $\mathrm{Na}(0)_{n}$ particles $^{12}$ that are also no longer composed of individual, $\mathrm{M}(0)$ atoms as is the case for assembled $(\mathrm{RH})_{n}$ subunits. CNT fails badly for these covalent, strong-bonded, $\mathrm{M}(0)_{n}$ systems too, as opposed to clusters of individual hydrocarbons, $(\mathrm{RH})_{n}$, inert gases $\left(\right.$ e.g., $\left.(\mathrm{Ar})_{n}\right)$, and water $\left(\mathrm{H}_{2} \mathrm{O}\right)_{n}$ or alcohols $(\mathrm{ROH})_{n}$, where CNT has proven to be applicable. ${ }^{12}$ Even there for simple $(\mathrm{MeOH})_{n}$ or ostensibly even simpler $(\mathrm{Ar})_{n}$ for example, the CNT-computed nucleation rates can differ from experimental measurements by $10^{6}$ to $10^{11-13}$, respectively, ${ }^{12,33}$ although nucleation rate measurements can be problematic even for the simplest systems. Even for the best systems, agreement within $\pm 10^{1-2}$ is considered a success, with errors of $10^{6-10}$ between CNT calculated and experimental nucleation rates not uncommon. ${ }^{12,16,34,35}$

Noteworthy here is that a $S_{n}$ sol on which the 1950 model is based, and its relatively strong $\mathrm{S}-\mathrm{S}$ bonds, is not a system where CNT should have been applied ${ }^{2}$ one can now say-while also acknowledging our additional 70 years of knowledge in fairness to those early researchers and as discussed more in our Part I review. ${ }^{2}$ Moreover, in terms of realizing where CNT should or should not be applied, it helps to realize that CNT is a macroscopic to microscopic (but not atomic), "top-down" theory-again, really just a hypothesis expressed as one possible mathematical model-brought down to the size of a still large "droplet" via the capillary approximation. CNT theorists likely had no idea at the time if they would need to treat $1000 \mathrm{~s}$ of atoms, or $<10$ or (as it now appears) $\leq 3$ atoms ${ }^{17,26,27}$ in the "critical nucleus" of at least some strong bonding systems, borrowing CNT language for a moment. Even for $\mathrm{H}_{2} \mathrm{O}$, the best current computations suggest there are no more than 10-50 water molecules in the, therefore, $\left(\mathrm{H}_{2} \mathrm{O}\right)_{\sim 10-50}$ "critical cluster", ${ }^{36,37}$ Those DFT studies also bear on the "strong" vs. "weak" bonded classifications of nucleating species noted above: increasing the $\Delta H_{\text {binding }}$ in $\left(\mathrm{H}_{2} \mathrm{O}\right)_{n}$ by $\sim 0.5 \mathrm{kcal} \mathrm{mol}^{-1}$ leads to a $\sim 10^{10}$ increase in the nucleating rate. ${ }^{37}$ DFT computational studies provide further evidence on the failings of several of the approximations made in CNT. ${ }^{36,37}$ 
Meriting mention here is a 2015, 557-page Faraday Discussions by multiple experts presenting their work and understanding of mostly crystal nucleation, entitled "Nucleation-A Transition State to the Directed Assembly of Materials". ${ }^{11}$ CNT of homogeneous solutions or gels is used repeatedly to try to explain data presented at that meeting. Yet, the Concluding Remarks section ${ }^{38}$ states that "classical nucleation theory does not adequately explain the crystal nucleation process". Also valuable is a 2016 review of nucleation in the journal Crystal Growth and Design ${ }^{13}$ noting, overall, that non-classical pathways are gradually replacing CNT as the explanation for nucleation in crystal formation. ${ }^{13}$ Examples of non-classical nucleation pathways are those involving prenucleation clusters (PNCs), a topic we will return to later while seeing that Turkevich made important suggestions even back in 1951 about what we now call PNCs.

What is needed to move our understanding of nucleation forward is not more attempts to tweak the approaching 100-years old theory/mathematical model that we know as CNT. Instead, we hypothesize that needed, first, are better, faster, more precise ways to experimentally monitor nucleation kinetics and mechanism(s) in real time for a variety of systems across nature. Even in atmospheric chemistry where nucleation is heavily researched, the minimum size nucleus presently observable is $\sim 1.5-3.0 \mathrm{~nm}$, so $\sim 0.5-2.0 \mathrm{~nm}$ above what some find as a $\sim 1.0 \mathrm{~nm}\left(\mathrm{H}_{2} \mathrm{O}\right)_{\sim 4}$ "critical cluster" size. ${ }^{12}$ Second, new, better theories that treat the kinetics problem we know as nucleation will be needed. We refer the interested reader to $\mathrm{B}$. Garrett and co-workers computational efforts and scholarly, well-referenced, insightful papers. ${ }^{36,37}$

In short, the evidence strongly suggests that CNT should not be applied to any strongly bonding systems where the identity of the associating units are lost and where high supersaturations look to be involved, such as sulfur $\left(\mathrm{S}_{n}\right)$ as in the original 1950 model $^{1}$ or metal $\left(\mathrm{M}_{n}\right)$ nanoparticles, ${ }^{2}$ as two examples. LaMer notes in his 1950 paper $^{1}$ that his model was developed "in conjunction with modern theories of phase transitions", 1 that is, in conjunction with CNT. ${ }^{22-24}$ The 1950 model assumes that CNT is correct (as one might expect for the times, CNT being reported 24 years prior ${ }^{21-25}$ to the 1950 paper $^{1}$ ) and starts with nuclei of radius $x$ from which growth is then postulated to start, as illustrated in Fig. 2. An underappreciated point here is that the 1950 model does not address nucleation directly. Instead, the 1950 model assumes a hypothetical form of nucleation-specifically instantaneous/burst nucleation-so that the mathematics of the growth model could be developed. As oddly as it sounds, the (assumed) "burst nucleation" model is actually not a physically or experimentally based nucleation model. Instead, it is really just a mathematical model of growth as documented in our Part I review. ${ }^{2}$ The irony here is that many of the citations of 1950's model cite it for its "instantaneous or burst nucleation" feature, that is, cite it if for an assumption in the model: the assumed, "word-only" postulate about nucleation. Those citations are often trying to explain how a relatively narrow particle-size distribution can or has been formed-claiming near-monodisperse particle formation is due to the-again assumed-initial burst of particles that, in the burst-nucleation postulate, would lead to the complete separation of nucleation and growth in time. But, that "instantaneous/burst nucleation" postulate ultimately leads back to CNT that, as detailed in this section, is ultimately a false foundation for a large fraction of the $c a .1761$ ( 90\%) out of the total citations ${ }^{2}$ of the 1950 paper. $^{1}$

Finally, it is useful to mention here that within CNT, the formation of the critical nucleus was historically believed to be possible and thus occur according to fluctuation theory. Fluctuation theory is described in the literature as "the process...governed by the probability of the occurrence of the least probable of these fluctuations, that is, the critical fluctuation, which, in terms of the more familiar nucleation theory, is known as the critical nucleus." 39 Under CNT, the general "rate equation" is given ${ }^{10}$ as eqn (2),

$$
J=A \times \exp \left(\frac{-\Delta G_{\text {crit }}}{k_{\mathrm{B}} T}\right)
$$

where $J$ is described as the "rate" of nucleation, $A$ is a general constant, $\Delta G_{\text {crit }}$ is the Gibbs free energy change for the formation of the "critical nucleus", $k_{\mathrm{B}}$ is the Boltzmann constant, and $T$ is temperature. The component $\exp \left(-\Delta G_{\text {crit }} / k_{\mathrm{B}} T\right)$ from eqn (2) is described in the literature as "the probability that a spontaneous fluctuation will result in the formation of a critical nucleus." 40 Hence, fluctuation theory is an underlying component of classical nucleation theory, specifically the postulate of how the statistically improbable "critical nucleus" might possibly be formed and despite its occupation of the highest free-energy point back in Fig. 1. As early a 1951 Turkevich questioned $^{5}$ the idea of a spontaneous/"burst" formation of all the nuclei at once as being statistically too improbable and therefore making little chemical sense. A referee commented that it would be of interest to have a modern computation and analysis of the probability of nucleation according to the fluctuation theory hypothesis, a point with which we agree.

One final point here is the CNT-based "rate" of nucleation does not change with progress of the reaction, but instead is a constant once it commences-thereby implied to be a chemically and mechanistically implausible zero-order process as already noted. Literature reviews on CNT confirm this with the statement that the "the rate of nucleation, $J$, is defined as the rate of growth of the critical nucleus" 41 (italics have been added). What this somewhat confusing statement is implying is that evidence for the putative critical nucleus itself is not possible, only its growth is observable. Again, this is just what H. Reiss noted in his aforementioned statement that "the nuclei themselves are never observed", ${ }^{28}$ at least when one is speaking of the "critical nucleus" as defined by CNT.

\subsection{The 1950 mathematical model en route to the first explanation in the literature of how monodisperse particles might possibly be formed}

An examination of the 1950 model leads to Fig. 2 that helps illustrate that mathematical model. ${ }^{2}$ 


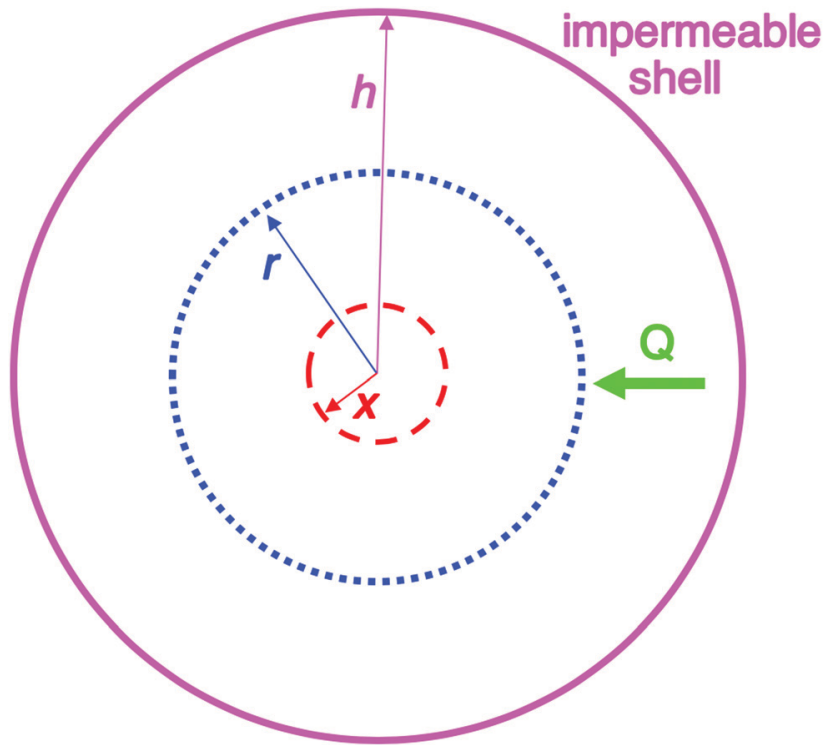

Fig. 2 Schematic illustration of the 1950 model consisting of a spherical volume with radius $h$ (solid pink circle), having a concentric spherical nucleus with radius $x$ (red dashed circle). In this model, radial growth occurs on the surface of the concentric spherical nucleus upon arrival of diffusing sulfur. $Q$ is the flux of diffusing sulfur through any concentric spherical shell with radius $r$ (blue dotted circle) within the sphere of impermeable shell (pink solid circle). Reproduced with permission from ref. 2. Copyright 2019 American Chemical Society.

Instantaneous nucleation as assumed by $\mathrm{CNT} /$ fluctuation theory is expressed in the instantaneous formation of nuclei with radius of size $x$ as shown in Fig. 2. Growth is postulated to occur on the surface of each nuclei at the same rate, resulting in monodisperse particles. A full list of the assumptions used to establish the 1950 model can be found in our Part I review. ${ }^{2}$ The important insight is ref. 2 ". . .the LaMer model is a growth model, one that says nothing about nucleation past making the assumption of 'instantaneous/burst' nucleation as was necessary at the time for developing the growth model."2 The key point is that "Having all of the nuclei there instantaneously in uniform size $x$ then allowed a mathematical model for growth in this first, creative attempt to try to understand how "monodisperse' particles could possibly be formed.",2

\subsection{Phase change or particle-formation models available in the literature since 1939}

In order to understand and put into historical perspective what follows, it is necessary to be aware of the mathematical models (and then the experimentally based chemical mechanisms, Section 1.6, vide infra) that have appeared in the literature to try to account for phase change kinetics data-or, in the case of the 1950 model, for particle-formation "phase-change" kinetics data.

The Avrami ${ }^{42-44}$ and later $\mathrm{KJMA}^{45,46}$ and Erofe'ev (A-E) ${ }^{47}$ are early mathematical/semi-empirical models in the literature from 1939 to 1946 striving to account for often sigmoidalshaped phase-change kinetics throughout nature, the Avrami model used originally to analyze the crystallization of fat. ${ }^{42-44}$ There are at least $\geq 12$ variants of the original Avrami model as workers strived to refine the model and deal with issues and problems inherent to these non-mechanistic models (see Table S1 in the ESI $\dagger$ elsewhere that tabulates and provides references to solid-state kinetics models ${ }^{48}$ ). Such a proliferation of derivative models with additional parameters, often striving to provide physical meaning and sense to the model, are a hallmark feature of models in trouble from the outset.

Most recently in 2017, a modified-KJMA (M-KJMA) ${ }^{49}$ was proposed to "ensure rate constants are intrinsic to the sample and reaction conditions, not the specific technique of measurement." 49 The assumptions behind and issues with these models are discussed elsewhere ${ }^{50,51}$ and need not be rehashed here, save to emphasize four key points: ${ }^{50,51}$ (i) all the models in Table 1 assume instantaneous/burst nucleation no matter if they are postulated to occur instantaneously at $t=0$ or for example at some later, predetermined time, $t=t_{0}$ (known as "sporadic" as opposed to "instantaneous" nucleation in the Avrami-model literature ${ }^{42-47,49}$ ); (ii) hence these mathematical

Table 1 Historical summary of mathematical/semi-empirical models for phase transitions (or sulfur sol formation, in the case of the 1950 model)

\begin{tabular}{|c|c|c|c|}
\hline Year & Person & Model & Resultant equation \\
\hline 1939 & Avrami $^{42-44}$ & Mathematical; semi-empirical & $\begin{array}{l}\ln (1-\alpha)=-(k t)^{n} \\
\text { Equivalently, } \alpha(t)=1-\exp \left\{-[k t]^{n}\right\}\end{array}$ \\
\hline
\end{tabular}

$[\alpha=$ extent (fraction) of reaction; $k=$ a rate parameter; $n=$ the "Avrami exponent"]

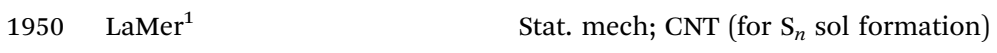

$\left[x=\right.$ nucleus radius; $\mathrm{ss}=$ supersaturation; $D=$ diffusion coefficient; $\rho=($ bulk $) \mathrm{S}_{n}$ density; $h=$ sphere radius $]$

$\frac{\mathrm{d}\left(x^{2}\right)}{\mathrm{d} t}=\left[C_{\mathrm{ss}}-C_{\mathrm{s}}(t)\right] \times \frac{2 D}{\rho}-\left(2 D / h^{3}\right) x^{3}$

Word version: burst nucleation from supersaturated solution, then diffusion-controlled growth

2017 Martin (M-KJMA) $\quad$ Mathematical; semi-empirical

[M stands for "Modified"; $k_{\mathrm{A}}=$ rate parameter; $n=$ Avrami exponent; $k_{\mathrm{A}}$ is postulated to be composed of $k_{\mathrm{A}}=\left[\frac{\nu_{\mathrm{pb}}}{g{ }^{1 / 3}}\right]$, where

$\nu_{\mathrm{vp}}=$ velocity of the phase boundary; $g=$ geometric factor; $a_{\mathrm{c}}=$ anisotropy correction; $n^{\prime}=$ anisotropy corrected dimensionality exponent; $V=$ volume] 
models say nothing about nucleation; instead, are really growth models post some presumed, undetailed nucleation events of unknown kinetics and unknown molecularity; (iii) the Avrami exponent, $n$, is a convolution of the dimension in space that the growth takes place in plus a parameter related to the assumed time dependence of the nucleation function. ${ }^{42-47,49}$ Hence and unfortunately, the Avrami exponent parameter, $n$, has been destined to failure despite $>80$ years of literature trying to deconvolute and interpret nucleation and growth convoluted from the start into the single Avrami parameter, $n$. Additionally, (iv) the one $k$ in the Avrami model is not a rate constant, as it is not defined by a balanced equation as rate constants must be. Instead it is a composite rate parameter that is a convolution of a linear diffusional growth $\left(k_{\mathrm{g}}\right)$ and some assumed, for example zero-order kinetics, linear nucleation rate parameter $(J)$. As a result, deconvolution of anything resembling actual nucleation from growth using the Avrami and related equations continues to be hopelessly confused despite years of literature trying to clarify things and make these models physically valid. For example, how can one possibly talk rationally and kinetically about the two processes of nucleation and growth when you have only one rate parameter, $k$ ? You can't, at least not directly-nor easily nor clearly.

The reader wishing to learn more and make up their own mind about whether or not to use these models for their phase changes including particle formation is referred to other discussions, including a recent Comment, ${ }^{52}$ then a Response, ${ }^{51}$ that debates if the M-KJMA equation vs. the 1997 2-step mechanism $^{3}$ discussed in the next section as ways to analyze initially particle-formation reactions. A related, valuable paper from Prof. G. Lente and R. Szabó shows ${ }^{53}$ that a stochastic kinetics approach to the 1997 2-step mechanism in the next section aligns well with the deterministic kinetics approach that originally discovered that 2 -step mechanism, ${ }^{3}$ all as Kurtz' theorem ${ }^{54}$ connecting stochastic and deterministic kinetics requires.

\subsection{A brief summary of particle-formation mechanisms available since 1952}

Table 2 is a summary in historical order of particle-formation mechanisms that at least we are aware of that have been generalized to an "A, B, C..." species form, ${ }^{55-63}$ as kineticists strive to do in the hope that their chemical mechanisms can be seen in more general form and, ideally, therefore applied more broadly. We have also included Turkevich's word-only organizer mechanism because it can be seen as an early version of the Prenucleation Cluster concept idea, vide infra. Reviews covering other, important contributions to particle formations, ${ }^{14}$ as well as to related topics of protein particle formation (aggregation), ${ }^{64}$ are available to the interested reader (please also see the many references provided in these reviews to the excellent work of the many authors cited ${ }^{14,64}$ ) in addition to the references covered in Table 2 .

Entry 1 in Table 2 is of considerable historical interest because it cites the $1952^{4}$ chemical mechanism LaMer was considering for how one chemically might get to the postulated "burst nucleation". LaMer based his proposed mechanism, as one would expect for the time in 1952, on the assumption of thermodynamic control of, therefore, reversible reactions where he envisioned that "...the embryos of the type $\mathrm{B}_{i}$ are continually forming and disappearing by the reverse processes of dissociation". Interesting is that he says that for the initial $m \mathrm{~A}_{1} \times \mathrm{B}_{m}$ process " $m$ is usually 2 ", ${ }^{4}$ but then that for the proposed, reversible step $\mathrm{B}_{(i-1)}+\mathrm{A}_{1} \rightleftharpoons \mathrm{B}_{i}$, " $i$ in the case of water vapor condensation is about $80-i . e$. this reaction is of the 80th order". 4 The 'rate', $J$, of this process according to eqn (2) and the energetics according to Fig. 1, vide supra, were never reconciled with this proposed, exorbitant 80th reaction order, kinetics with little precedent to this day according to our knowledge or according to a search of the literature. ${ }^{65}$

Of further interest is that LaMer reported in that same 1952 paper $^{4}$ an interesting log-log plot of the log $\left[\mathrm{Ba}^{2+}\right]\left[\mathrm{SO}_{4}{ }^{2-}\right]$ ionic mean molality vs. the log (lag time) to the appearance of turbidity, reproduced below as Fig. 3 .

Based on the data in Fig. 3, LaMer proposed a mechanism ${ }^{4}$ consisting of $3 \mathrm{Ba}^{2+}$ reacting with $3 \mathrm{SO}_{4}{ }^{2-}$ (as his explanation for the overall $\sim 6$ th order reaction to observable precipitate) where "all of the preceding steps are assumed in a quasi-equilibrium state." Interestingly and again as one would expect for the time, LaMer thought that he had observed the critical nucleus of CNT. Hence, he interpreted the overall reaction as being 7 th (not 6th) order, as the critical nucleus would then need to add one more ion $\left(\mathrm{Ba}^{2+}\right.$ or $\left.\mathrm{SO}_{4}{ }^{2-}\right)$ to be consistent with the postulated, CNT-based rate-determining step. LaMer insightfully concluded his paper with "This new attack on the size of the nucleus merits further refinement and extension to new systems", 4 a statement true today given that definitive studies of the kinetics and mechanisms of nucleation are often still lacking.

Of course, we now can hypothesize that these early researchers were actually observing what we would now call the first observable clusters ${ }^{17}$ (really particles) from "burst (autocatalytic) growth"-not burst nucleation. Those particles had to be at least $\sim 100$ microns to be seen by the naked eye, ${ }^{68}$ so something on the order of $\left(\mathrm{BaSO}_{4}\right)_{n}$ where $n \sim 6 \times 10^{15}$, one can estimate. ${ }^{69}$ These would then be large particles way beyond the nucleation stage-the result of (burst) autocatalytic growth. Even a Tyndall beam, the best light scattering technique at the time, could only observe particles of $\sim 0.2$ microns $(\sim 200 \mathrm{~nm})$ diameter, hence $n \sim 4 \times 10^{7}$, still relatively large particles almost surely way past the nucleation stage. Additionally, we also know from mathematical analysis of the first, second, and third ("jerk") derivatives

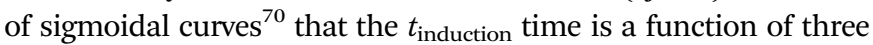
variables: the nucleation rate constant, the growth rate constant, and the starting monomer concentration (e.g., $k_{1}, k_{2}$, and $[\mathrm{A}]_{0}$ in the case of the 2-step mechanism in Table $2^{70}$ ).

Fascinating here as well is Turnbull's 1953 publication $^{71}$ where he thoroughly evaluates the claims of LaMer's 1952 paper and questions the assumption that $1 / t_{\text {induction }}$ is proportional to homogeneous nucleation. In fact, Turnbull convincingly demonstrates that nucleation in the $\left(\mathrm{BaSO}_{4}\right)_{n}$ precipitation system is heterogeneous, ${ }^{71}$ almost surely due to dust contamination and its kinetic effects-heterogeneous nucleation being the way around anything approaching 
Table 2 Historical summary of pseudo-elementary step, minimum mechanisms for particle formation (or crystal formation in the case of Tsapatsis' and co-worker's mechanism) that are also provided in generalized, and hence potentially more broadly applicable, "A, B, C..." kinetics form

\begin{tabular}{|c|c|c|c|}
\hline 1952 & V. K. LaMer ${ }^{4}$ & $\begin{array}{l}m \mathrm{~A}_{1} \rightleftharpoons \mathrm{B}_{m} \\
\mathrm{~B}_{m}+\mathrm{A}_{1} \stackrel{F}{\rightleftharpoons} \mathrm{B}_{m+1} \\
\mathrm{~B}_{(i-1)}+\mathrm{A}_{1} \rightleftharpoons \mathrm{B}_{i} \\
{\left[\mathrm{~A}_{1}=\text { "kinetically independent unit }\right.} \\
2 \text {, whereas } i \text { in the case of water } \mathrm{v}\end{array}$ & $\begin{array}{l}\text { Stepwise bimolecular addition leading to "burst nucleation" (NB: } \\
\text { "diffusion-controlled growth", was not included }{ }^{4} \text { as part of the scheme } \\
\text { provided) } \\
\text { of phase } \mathrm{A}^{\prime \prime}, \mathrm{B}_{i}=\text { "embryo of phase B containing } i \text { units", and " } m \text { is usually } \\
\text { por condensation is about } 80-\text { i.e., this reaction is of the 80th order"] }\end{array}$ \\
\hline 1997 & Finke, Watzky ${ }^{3}$ & $\begin{array}{l}\mathrm{A} \stackrel{k_{1}}{\longrightarrow} \mathrm{B} \\
\mathrm{A}+\mathrm{B} \stackrel{k_{2}}{\longrightarrow} 2 \mathrm{~B} \\
{[\mathrm{~A}=\text { metal precursor, } \mathrm{B}=\text { growing, }}\end{array}$ & $\begin{array}{l}\text { Slow, kinetically continuous nucleation followed by typically fast, } \\
\text { explosive autocatalytic surface growth. Important to note is that higher } \\
\text { order } n \mathrm{~A} \stackrel{k_{1}}{\longrightarrow} n \mathrm{~B} \text { nucleations are covered by (i.e., can be hidden in) the } \\
\text { pseudo-elementary first step, as the subsequent discovery of second- } \\
\text { order and termolecular nucleations demonstrates. }{ }^{17,26,27} \\
\text { metal(0) particle] }\end{array}$ \\
\hline 2005 & $\begin{array}{l}\text { Besson, Finney, } \\
\text { et al. }{ }^{56-58}\end{array}$ & $\begin{array}{l}\mathrm{A} \stackrel{k_{1}}{\longrightarrow} \mathrm{B} \\
\mathrm{A}+\mathrm{B} \stackrel{k_{2}}{\longrightarrow} 2 \mathrm{~B} \\
\mathrm{~B}+\mathrm{B} \stackrel{k_{3}}{\longrightarrow} \mathrm{C} \\
\mathrm{B}+\mathrm{C} \stackrel{k_{4}}{\longrightarrow} 1.5 \mathrm{C} \\
{[\mathrm{A}=\text { metal precursor, } \mathrm{B}=\text { small pa }}\end{array}$ & $\begin{array}{l}\text { Evidence for a } 4 \text { th step of autocatalytic agglomeration where the smaller } \\
\text { particles, B, and larger particles, C, agglomerate with a separate, } k_{4} \text {, rate } \\
\text { constant }\end{array}$ \\
\hline 2005 & $\begin{array}{l}\text { Drew, Katsoulakis, } \\
\text { Tsapatsis }^{59}\end{array}$ & $\begin{array}{l}\mathrm{A} \stackrel{k_{1}}{\longrightarrow} \mathrm{B} \\
\mathrm{B} \stackrel{k_{2}}{\longrightarrow} \mathrm{C}_{1} \\
\mathrm{~B}+\mathrm{C}_{i} \stackrel{K_{i}}{\longrightarrow} \mathrm{C}_{i+1} \\
\mathrm{~A}+\mathrm{C} \stackrel{K_{4^{\prime}}}{\longrightarrow} \mathrm{C}_{i+1} 1.5 C \\
{[\mathrm{~A}=\text { primary colloidal particles; }}\end{array}$ & $\begin{array}{l}\text { A proposed mechanism for crystal growth by aggregation of meta-stable } \\
\text { nanoparticle precursors, one used with Population-Balance Modeling. } \\
\text { Three alternative mechanisms were tested; shown is the author's } \\
\text { preferred Mechanism II. }\end{array}$ \\
\hline
\end{tabular}

2014 Kent, Mondloch, et al. ${ }^{60}$

$$
\begin{array}{ll}
\mathrm{A} \stackrel{k_{1}}{\longrightarrow} \mathrm{B} & \begin{array}{l}
\text { Evidence for a new 4th step } \\
\text { larger particles, C, growth w }
\end{array} \\
\mathrm{A}+\mathrm{B} \stackrel{k_{2}}{\longrightarrow} 2 \mathrm{~B} & \\
\mathrm{~B}+\mathrm{B} \stackrel{k_{3}}{\longrightarrow} \mathrm{C} & \\
\mathrm{A}+\mathrm{C} \stackrel{k_{4^{\prime}}}{\longrightarrow} 1.5 \mathrm{C} & \\
{[\mathrm{A}=\text { metal precursor, } \mathrm{B}=\text { smaller particles, } \mathrm{C}=\text { larger particles }]}
\end{array}
$$

2017 Karim, et al. ${ }^{63}$

$\mathrm{A}+\mathrm{L} \rightleftharpoons \mathrm{A} \cdot \mathrm{L}$

$\mathrm{A} \stackrel{k_{1}}{\longrightarrow} \mathrm{B}$

$\mathrm{A}+\mathrm{B} \stackrel{k_{2}}{\longrightarrow} 2 \mathrm{~B}$

Evidence for a new 4 th step of secondary autocatalytic growth where larger particles, C, growth with a separate, $k_{4^{\prime}}$, rate constant.

$\mathrm{B} \cdot \mathrm{L} \rightleftharpoons \mathrm{B}+\mathrm{L}$

[A = metal precursor, $\mathrm{B}$ = growing particle, $\mathrm{A} \cdot \mathrm{L}$ = precursor with ligand, $\mathrm{B} \cdot \mathrm{L}$ = growing particle with ligand; bh ligand steps have associated $K_{\text {eq }}$ equilibrium constants]

2019 Handwerk, Shipman, Whitehead, Özkar, et al. ${ }^{61,62}$

$$
\begin{aligned}
& \mathrm{A} \stackrel{k_{1}}{\longrightarrow} \mathrm{B} \\
& \mathrm{A}+\mathrm{B} \stackrel{k_{2}}{\longrightarrow} \mathrm{C} \\
& \mathrm{A}+\mathrm{C} \stackrel{k_{3}}{\longrightarrow} 1.5 \mathrm{C}
\end{aligned}
$$

A new, 3-step mechanism, discovered by disproof-based mechanismenabled population-balance modeling, in which the $\mathrm{A}+\mathrm{B} \stackrel{k_{2}}{\longrightarrow} 2 \mathrm{~B}$ pseudo-elementary step of the 1997 2-step mechanism is expanded into two growth steps (or, alternatively, and as actually discovered ${ }^{61,62}$ the 2nd and 3rd steps of the 2014 4th mechanism are combined into a single, new 2nd step). Eleven alternative mechanisms were disproved. Quantitative fitting of particle-size distributions (PSDs) was demonstrated, including the PSD shape.

[A = metal precursor, $\mathrm{B}=$ smaller particles, $\mathrm{C}=$ larger particles $]$ 


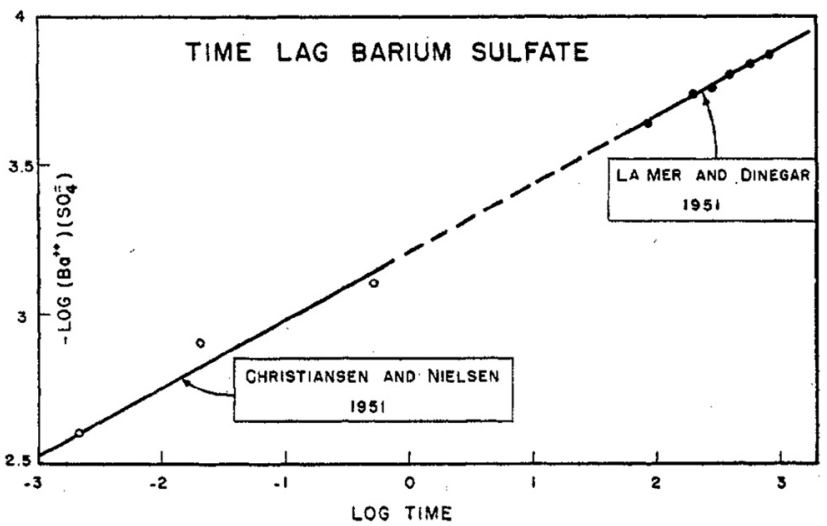

Fig. 3 Reproduction of Fig. 5 from ref. 4. The log of ionic mean molality for $\left[\mathrm{Ba}^{2+}\right]\left[\mathrm{SO}_{4}{ }^{2-}\right]$ is plotted against the log (lag time) data from both Christiansen and Nielsen's 1951 study ${ }^{66}$ and LaMer and Dinegar's slightly later, also 1951 study ${ }^{67}$ Here the lag time is the time until the turbidity first became observable (by eye in Christiansen and Nielsen's study ${ }^{66}$ and by a Tyndall beam in LaMer and Dinegar's study. ${ }^{67} \mathrm{BaSO}_{4}$ particles were visible by eye once the condensation point was reached and large particles precipitated and fell to the bottom of the reaction flask. The $y=m(x)+$ $b$ line fits $-\log \left[\mathrm{Ba}^{2+}\right]\left[\mathrm{SO}_{4}{ }^{2-}\right]=0.22 \log t+b$, that is, $-4.5 \cdot \log \left[\mathrm{Ba}^{2+}\right]\left[\mathrm{SO}_{4}{ }^{2-}\right]=$ $\log \left(\left[\mathrm{Ba}^{2+}\right]\left[\mathrm{SO}_{4}{ }^{2-}\right]\right)^{-4.5}=\log t+4.5 \cdot b$. This in turn corresponds to the plot for an integrated rate law for a $n\left(\mathrm{BaSO}_{4}\right) \rightarrow\left(\mathrm{BaSO}_{4}\right)_{n}$ rate law, where the math shows that $n-1=4.5$, so $n=4.5+1=5.5(\approx 6$, upon rounding up), consistent with LaMer's report of a net $\sim 6$ th order reaction. ${ }^{67}$ Reproduced with permission from ref. 4. Copyright 1952 American Chemical Society.

something like a putative "80th order reaction", one can say in reference back to LaMer's comment about water nucleation. Turnbull also points out that growth is not limited "by diffusion, but by a process occurring at the crystal-solution interface."71 Turnbull observed sigmoidal growth curves in his studies, curves fit using an Avrami-type equation. ${ }^{48,51}$ We now know that those sigmoidal curves will, therefore, ${ }^{48}$ also be well-fit by a chemical equivalent ${ }^{48}$ of the Avrami mathematical and semi-empirical equation, specifically by the 2-step mechanism back in Table 2 corresponding to slow, continuous nucleation and autocatalytic surface growth., ${ }^{3,48}$

Hence, as early as 1951 , others ${ }^{5,6,71}$ in the literature were aware of (i) the limitations of classical nucleation theory, (ii) the issues associated with only using turbidity measurements, (iii) the omnipresence of dust effects and heterogeneous nucleation, all while they were also (iv) collecting experimental evidence against the 1950 model of burst nucleation and diffusion-controlled growth. Most importantly, (v) both LaMer and Turnbull had experimental evidence disproving a fluctuation/CNT theory type of high-order nucleation for the $\left(\mathrm{BaSO}_{4}\right)_{n}$ precipitation system, and Turnbull had strong evidence for heterogeneous nucleation and non-diffusion-controlled growth.

If follows that these early studies of the kinetics of $\left(\mathrm{BaSO}_{4}\right)_{n}$ precipitation are actually still of considerable current interest and, hence, merit careful reanalysis. If we discard CNT for interpreting these 1950's results on the belief that $\left(\mathrm{BaSO}_{4}\right)_{n}$ is a strong-bonding system, vide supra, then the straightforward, simplest interpretation of these early chemical kinetics is that they indicate a rate law third-order in $\mathrm{BaSO}_{4}$. That is, a rate law
$\alpha\left[\mathrm{BaSO}_{4}\right]^{3}$ is implied, in turn suggesting a rate-determining step for the observation of observable precipitate of just $3 \mathrm{BaSO}_{4} \rightarrow$ $\left(\mathrm{BaSO}_{4}\right)_{3}$. Of considerable interest here is that an analogous, low molecularity kinetically effective nucleus (KEN) ${ }^{17}$ of just $2-3$ has now been documented kinetically for several strongbonding nucleating systems in nature. ${ }^{17,26,27}$ While it is not at all clear that the net $3 \mathrm{rd}$ order, $\left[\mathrm{BaSO}_{4}\right]^{3}$ kinetics correspond to a $\mathrm{KEN}=3$ in that system-because considerable growth has had to occur before any "nucleus" becomes visible as a much larger particle (pretty much exactly as Turnbull insightfully cautioned ${ }^{66}$ - a deeper, modern look at the $\left(\mathrm{BaSO}_{4}\right)_{n}$ system, Turnbull's 1953 study and Fig. 3 above are warranted.

The important historical example of $\left(\mathrm{BaSO}_{4}\right)_{n}$ precipitation also documents that LaMer was aware of the need for chemical kinetics and mechanistic investigations, that he too knew about heterogeneous versus homogeneous nucleation, ${ }^{4}$ and that he did provide a possible chemical mechanistic alternative in 1952. Hence, researchers could have-and should have-not ignored but instead compared and contrasted the $\left(\mathrm{BaSO}_{4}\right)_{n}$ mechanistic model to the 1950 mathematical model and its postulate of burst nucleation and diffusion-controlled growth. While with 70 years of hindsight one can say it is unfortunate that the above $\mathrm{BaSO}_{4}$ investigations and the proposed 1952 chemical mechanism in Table 2, entry 1 , were not part of the classic 1950 paper. ${ }^{1}$ However, as we noted in our Part I review ${ }^{2}$ "LaMer and his co-workers labored in a pioneering and creative way, both theoretically and experimentally" and "that they labored in much different times, where physical methods were limited, modern computation power did not exist, and classical nucleation theory was a relatively new, exciting, and probably seductive theory that likely seemed compelling at the time.",

1.6.1. Turkevich's 1953 “Organizer” pathway: the early "Prenucleation Cluster" mechanism. Turkevich was researching $\mathrm{Au}(0)_{n}$ particle formation ${ }^{5,6}$ around the same time as LaMer was studying $(\mathrm{S})_{n}$ sol formation and $\left(\mathrm{BaSO}_{4}\right)_{m}$ precipitation. In 1951, Turkevich found that his $\mathrm{Au}(0)_{n}$ size versus time plot showed exponential (sigmoidal) growth before leveling off, but that a "mechanism of this exponential law of growth must await further experimental studies of the growth reaction." ${ }^{, 5}$ However, it is clear that Turkevich believed that the 1950 model was not applicable to his $\mathrm{Au}(0)_{n}$ formation system. Specifically, Turkevich commented that fluctuation theory (and by extension classical nucleation theory) was in 1951 already 'a theory of great tradition', statement implying that he had disagreements with these theories.

After additional study, in 1953 Turkevich sought to explain the nucleation of $\mathrm{Au}(0)_{n}$ sols by postulating his own "Organizer" pathway, whereby he postulated "the nucleating agents gradually build up a chemical complex between themselves and gold ions, organizing the latter into a macromolecule (precursor of the nucleus). When the size of the macromolecular inorganicorganic polymer is sufficiently great, it will undergo a molecular rearrangement to produce the metal nucleus and oxidation products of the organizer.", 6 Turkevich postulated his "Organizer mechanism" for nucleation because of what he felt were unrealistic components of fluctuation theory, where, as he described it, 
"...the fundamental difficulty in building up a nucleus is the accumulation of a sufficiently large local concentration of atoms whose size is greater than just demanded by the stability of the particle." 6 Turkevich reasoned that such a build-up is unrealistic statistically because, he argued, that his gold sol nuclei "are about $30 \AA$ diameter and this would involve a fluctuation of the order of a million of gold atoms." 5 Hence, Turkevich proposed his "Organizer" model what he felt is as a more realistic alternative en route to assembling the atoms or molecules needed for nucleation. Anyone who has read Turkevich's work will likely be impressed with his efforts and beforetheir-time insights.

In modern-day terms, we can recognize Turkevich's creative "Organizer" hypothesis as analogous to-and really the first instance of-the currently popular concept of "prenucleation clusters", namely the assembly of reaction precursors into large macrostructures with longer-range order that are on-path, kinetically viable nuclei able to undergo facile growth. ${ }^{72}$ The concept of prenucleation clusters has been increasingly applied in the literature to the following systems: $\mathrm{H}_{2} \mathrm{SO}_{4}{ }^{73} \mathrm{CaCO}_{3},{ }^{72,74}$ homogeneous crystallizations, ${ }^{75} \mathrm{Na}_{2} \mathrm{~S}_{2} \mathrm{O}_{3},{ }^{76}$ heterogeneous protein crystallization, ${ }^{77}$ glass-type transitions in the solid-state, ${ }^{78-80}$ and most recently $\mathrm{Au}(0)_{n}$ nanoparticles. ${ }^{81}$ The allure of the prenucleation cluster concept so noted, one critical issue with prenucleation clusters is if the observed clusters are kinetically on, vs. off, path to the final product. The observable clusters may sometimes, and perhaps often, be too stable off-path clusters or clusters trapped in off-path kinetic bottlenecks. Noteworthy here is that that the famous mechanistic chemist and kineticist Professor Jack Halpern ${ }^{82}$ among others ${ }^{83,84}$ have emphasized that no measurement made under steady-state conditions can tell if a given "intermediate" is on- versus off-path. Pre- and/or post-steady-state kinetics measurements are required to differentiate those challenging-to-distinguish, two limiting kinetics interpretations. ${ }^{82-84}$ Nevertheless, at present the concept of prenucleation clusters remains an intriguing and exciting alternative hypothesis to CNT for many literature systems, a concept that merits additional attention and more extensive study by direct methods monitoring the kinetics of the observable clusters, including pre- and poststeady state kinetics measurements.

1.6.2. Other, 2-, 3- and two 4-step mechanisms for particle formation and subsequent agglomeration. It was not until 1997, almost 50 years after the 1950 model, that a chemical mechanism - that is, an experimentally determined, disproofbased, deliberately minimalistic mechanism-was described in the literature. ${ }^{3}$ That mechanism is the 2-step mechanism $\mathrm{A} \rightarrow \mathrm{B}$, slow continuous nucleation rate constant $k_{1}$, and $\mathrm{A}+\mathrm{B} \rightarrow 2 \mathrm{~B}$, autocatalytic surface-growth rate constant $k_{2}$, in Table 2, entry $\# 3^{3}$ and comes from the laboratories of the senior author of this review. The deliberately minimalistic and thereby obviously oversimplified 2-step mechanism-for particle formation reactions that often contain 1000 s or more of chemical steps $^{61,62}$ - has subsequently been used to quantitatively fit multiple types of particle formation/phase transition data, including homogeneous catalyst formation, ${ }^{85}$ heterogeneous catalyst formation, ${ }^{86}$ protein aggregation, ${ }^{87}$ adsorption in water treatment, ${ }^{88}$ and other sigmoidal nucleation and growth curves in nature. Disproof-based, again deliberately minimalistic (Ockham's razor-obeying) 3 -step, ${ }^{55}$ and two 4-step ${ }^{56-58,60}$ mechanistic models also exist as shown in Table 2. Noteworthy and likely to see much more emphasis is Professor A. Karim's important ligand-modified analog ${ }^{63}$ of the 2-step mechanism shown in Table 2, a mechanism that will come up again for further discussion in Section 5.4.3. Noteworthy here is that a combinatorial consideration of the individual pseudoelementary mechanistic steps in Table 2 along with just the precedented first, ${ }^{3}$ second, ${ }^{17}$ and net third-order ${ }^{26}$ nucleation yields 5 classes of mechanisms containing 96 reasonable, distinct mechanisms for consideration for one's particle formation reaction. ${ }^{61,62}$

The limitations of the 2-step (and 3-, and two 4-step) mechanistic models in Table 2 merit emphasis and have been published many times. ${ }^{48,60,89}$ Those limitations are required reading for anyone using these deliberately minimal mechanistic models. Those limitations ultimately derive from the toosimple and average-property results from the minimalistic model (e.g., size-averaged nucleation, $k_{1}$, and average autocatalytic growth, $k_{2}$, rate constants, as well as inability of at least the 2-step mechanism to describe anything beyond the average particle size $\left.{ }^{90}\right)$. The general species " $\mathrm{B}$ " and " $\mathrm{C}$ " are in some sense ill-defined as only smaller and larger particles, something that hides the underlying elementary steps such as monomer addition $\mathrm{A}+\mathrm{B}_{j} \rightarrow \mathrm{B}_{j}$, although this is taken care of in the population-balance modeling in the next section. As already briefly noted, a paper showing that the 2-step model appears to be the minimum chemical mechanism underlying Avrami-based equations has also been described. ${ }^{48}$ That said, use of the 2-step model is by no means accepted among at least one member of the solid-state kinetics community ${ }^{52}$ (see also ref. 51, 53 and 54) where the Avrami-based semi-empirical models back in Table 1 have historically dominated.

1.6.3. Minimum mechanistic schemes combined with population-balance modeling: mechanism-enabled population balance modeling. One exciting recent advance does merit mention for a proper perspective of the field before digging into the literature bearing on the 1950 model that will be examined in the sections that follow. That development is of what was coined as mechanism-enabled population-balanced modeling (ME-PBM) ${ }^{61,62}$ - that is, use of experimental, disproofbased mechanisms such as those in Table 2 to enable the initial formation (the nucleation), and then the growth and any agglomeration, of each particle in the particle formation process. Using ME-PBM, if one has an experimental mechanism of nucleation $^{17,26,27}$ along with a fit to one of the disproof-based mechanistic models in Table $2,{ }^{61,62}$ then it is now possible to fit particle size distributions (PSDs), including their shape, while extracting quantitative rate constant information from the kinetics-information-rich PSD. It is critical to have an experimentally determined nucleation mechanism to be able to start the particle formation process off correctly, as illustrated by an attempt to use CNT to start the particle formation process that, in 
turn, led to erroneous conclusions, even in the hands of a worldclass PBM expert. ${ }^{91}$

In at least the initial example, ${ }^{61,62}$ the ME-PBM was also able to contribute to the "inverse problem" of yielding a better idea of the "cause"-the mechanism-from observation of the "effects", namely the PSD. ${ }^{61,62}$ Indeed, that is how the new 3-step mechanism in Table 2 was discovered, via disproving 11 alternative mechanisms and their ME-PBMs en route to the new 3-step mechanism. ${ }^{61,62}$

Very excitingly, the new 3-step mechanism that was discovered can account for near-monodisperse particle formation by the paradigm-shifting finding that the smaller particles, B, grow faster and thereby catch up with the slower growing, larger particle C, that is, $k_{2}>k_{3}$ in the 2019 paper that is the last entry in Table 2. There is, in turn, no need for the postulate of "burst/instantaneous" nucleation in order to obtain (near) "monodisperse" PSDs. Hence, it would appear that the "smaller grow faster than larger particles" finding is the missing link needed for a paradigm shift away from 1950 postulate of instantaneous/burst nucleation. The ME-PBM's MATLAB code is posted on GitHub, hence freely available ${ }^{61,62}$ for others to use to test and their own particle formation systems and postulated mechanisms. The ability to predict PSDs for a known mechanism and input rate constants and concentration parameters is a notable "first" of the ME-PBM methodology, ${ }^{61,62}$ something that is it hoped will drive rational syntheses of narrower and narrower particle-size distributions.

A 2005 paper by Tsapatsis and co-workers ${ }^{59}$ and listed in Table 2 merits mention before ending this section. There, even though they were able to rule out only two other mechanisms, and did not fit any PSD data, that work is still a very valuable early precursor to ME-PBM. ${ }^{59}$ A paper that qualifies as ME-PBM in the polymer literature and from Waymouth's laboratories ${ }^{92}$ at Stanford also is recommended to anyone interested in applying ME-PBM to their own system. These and other related, recommended literature are discussed in the publications reporting ME-PBM. ${ }^{61,62}$

\section{Survey and selection of literature citing the 1950 model}

It is important that we present how we found and then sorted through the references that cite the 1950 model. That "Experimental" section is as follows: a Web of Science search was performed to start and revealed that Professor Victor LaMer's original $1950 \mathrm{~J}$. Am. Chem. Soc. paper ${ }^{1}$ has garnered 1953 citations (multiple literature searches were done over a multiyear period, with our final search being done and adding just a few more papers in March 2019). We then inspected all those papers with the goal of sorting them into two groups: those that provide just a reference to the 1950 model or just a "words-only" claim about the applicability of the 1950 model $v s$. those that provide new data plus those that just provide any depth of discussion or analysis at all (i.e., vs. just a citation of the 1950 model). This sorting was accomplished by reading first the abstract, then checking the details in the paper, and eventually then a at least cursory reading of the entire paper.

The inspection of those 1953 papers resulted in 192 papers (again as of our March 2019 cut-off time) that provide at least a discussion if not additional data bearing on the applicability of the 1950 model to the system at hand. The remaining 1761 papers $(\sim 90 \%)$ provide in our opinion little if any depth of discussion on the applicability of the 1950 model to the system at hand. Instead, $\sim 90 \%$ of the total papers we found just cite the 1950 model.

Next, the $\sim 10 \%$ (192 papers) located, that do provide at least some discussion of or data related to the applicability (or not) of the 1950 model (to the specific system in a given paper), were examined critically. Those papers are tabulated thematically with comments in a series of six summary tables: 28 papers on classic sulfur sol formation (covered in our Part I review, Table S1, ESI therein ${ }^{2}$ ); 13 papers on silver halide nanoparticles (the present Part II review, Table S1, ESI $\dagger$ ); 26 papers on semiconductor nanoparticles (Table S2, ESI $\dagger$ ); 69 papers on transition-metal nanoparticle formation (Table S3, ESI $\dagger$ ); 39 papers on oxide-based nanoparticles (Table S4, ESI $\dagger$ ); and 17 papers presenting alternative models or mechanisms compared to the 1950 model (Table S5, ESI $\dagger$ ), for a total of 164 papers examined in detail herein (plus 28 papers in our Part I review, ${ }^{2}$ accounting for the total of 192 papers).

We wish to emphasize that the reader wanting to reach their own at least preliminary conclusions is strongly urged to stop at this stage and read and study the 5 tables and 74 pages of ESI $\dagger$ before proceeding further. This is not a trivial point given that the senior author of the present review is responsible for 4 of the 11 total models or mechanisms in Tables 1 and 2 that compete with LaMer's 1950 model. That fact places him and his group in either a position of some arguably unavoidably bias or perhaps a position of some authority to write the present review-and practically speaking probably a bit of both. Many times we have thought that it would have been much better if the 1997 2-step mechanism back in Table 2 had been discovered back in 1926 or a bit thereafter rather than in $1997 .^{3}$ If it had been, then as argued in our Part I review ${ }^{2}$ “. . . the discovery of particle formation mechanisms would have been considerably accelerated..." because an experimental alternative to the LaMer theoretical model would have been available for competing consideration for the last 70 , and not just the last 23 years. A much earlier discovery of the 2-step mechanism by others would have also removed us from constantly being in an apparent if not real conflict-of-interest position vs. the 1950 model just because we discovered the first minimum mechanism ${ }^{3}$ that serves as an alternative to the 1950 model.

In what follows, we present a critical analysis-and to the best of our abilities an impartial, attempted unbiased analysis-of those additional 164 papers that cite or otherwise invoke the 1950 model. We also present the other, relevant literature required to understand or place in context those papers or our analysis. This Part II review along with our Part I contribution ${ }^{2}$ constitute the first comprehensive review of the 1950 model and its voluminous literature since the model's 1950 inception. ${ }^{1}$ The key, overarching 
question we aim to answer in what follows is whether or not there is compelling experimental evidence for "instantaneous nucleation" or "diffusion-controlled" growth in any of the 164 papers culled from the total literature citing the 1950 model. We start with $(\mathrm{AgX})_{n}$, and then move on to semiconductor nanoparticles such as $(\mathrm{CdS})_{n},(\mathrm{CdSe})_{n}$, or $(\mathrm{InP})_{n}$, because these systems are where the literature suggested that the 1950 model had the best experimental support.

\section{Formation of silver halide nanoparticles}

Nanoparticles of silver halides are of considerable interest because of their potential applications in many fields including photocatalysis, ${ }^{93}$ biosensing, ${ }^{94}$ and treating infections caused by microbacteria. ${ }^{95}$ Performance of silver halide nanoparticles in these applications depends on the particle size and sizedistribution, as well as the morphology and composition in the case of ternary silver halides. Hence, the synthesis of $(\mathrm{AgX})_{n}$ nanoparticles requires an understanding the nanoparticle formation process in order to control nanoparticle size and size-distributions.

Table S1 of the ESI $\dagger$ gives a summary review of thirteen selected papers reporting efforts to unravel the formation mechanism of silver halide nanoparticles. In what follows, key papers from Table S1 (ESI $\dagger$ ) are discussed as their evidence and results bear on either the support or disproof of the 1950 model for the specific case of silver halide nanoparticles.

\subsection{Early work on the formation of monodisperse silver halide particles}

An early 1947 study $^{96}$ reports the preparation of monodisperse silver sols by combining solutions of $\mathrm{Ag}^{+}$ions and $\mathrm{Cl}^{-}$ions. The transmittance (turbidity) of silver chloride sols was studied, and a first-order decay in the transmittance was observed in this early silver halide system.

Fifteen years later, a 1961 paper by Ottewill and Woodbridge $^{97}$ reported the preparation of silver bromide and silver iodide sols in water. Electron microscopy and higher order Tyndall spectra (HOTS) were used to examine the particle size and degree of dispersity. The formation of silver bromide sol from the decomposition of the complex ion, $\mathrm{AgBr}_{3}{ }^{2-}$, was followed by monitoring the optical density, Fig. $4 .{ }^{97}$ The authors contend that their data are "in agreement with LaMer's hypothesis." 97 However, the optical density versus time plot in one of the two curves in Fig. 4 has a small but detectable induction period that is hard to explain in terms of "burst nucleation". More kinetics early in the reaction are needed to understand the induction period and mechanism in this 1961 system that is still of interest. Controlling the dust content in this system should be one of the first orders of business.

In 1968, Insley and Parfitt ${ }^{98}$ reported the preparation of monodisperse $(\mathrm{AgCl})_{n}$ sols from the hydrolysis of allyl chloride in aqueous silver nitrate solution, monitored by conductance measurements, electron microscopy and electrophoresis. Shown

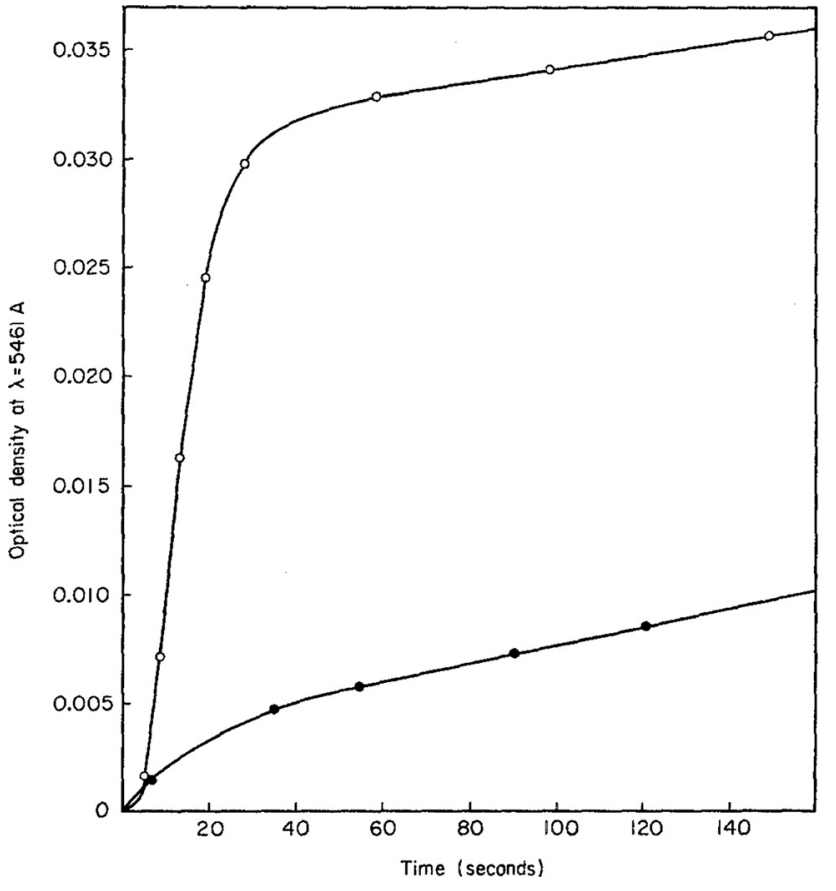

Fig. 4 (Fig. 7 in ref. 97.) Decomposition of the $\mathrm{AgBr}_{3}{ }^{2-}$ complex, by the addition of distilled water (open circles) or $10^{-9} \mathrm{M}$ silver nitrate solution (filled circles), to form $(\mathrm{AgBr})_{n}$. Reproduced with permission from ref. 97. Copyright 1961 Elsevier.

in Fig. 5 are the conductivity measurements, Fig. $5 \mathrm{a},{ }^{98}$ and the "critical ion product" (defined by the authors as $\mathrm{K}_{\mathrm{ss}}=\left[\mathrm{Ag}^{+}\right]\left[\mathrm{Cl}^{-}\right]$), for the precipitation of silver chloride from the equilibrium solution) from the fraction of the precipitated silver chloride $v s$. time data combined with the final size data, Fig. $5 \mathrm{~b}$.

In Fig. 5a, the conductance versus time plot shows an initial linear increase in conductivity due to release of hydrochloric acid from the slow $\mathrm{Cl}^{-}$abstraction and hydrolysis reaction of allyl chloride assisted by $\mathrm{Ag}^{+}$. The subsequent slow deviation from the linear conductivity was attributed to the solution having become saturated with respect to $\mathrm{AgCl}$ precipitation. No dramatic change that could be attributed to "burst" nucleation is observed. In Fig. 5b, an extrapolation back to zero of the fraction-precipitated versus time plot gives a non-zero time-axis intercept, suggesting an induction period is present. The authors concluded that their results are inconsistent with the 1950 model because the critical ion product changes quickly in the zero-point-of-charge region. ${ }^{98}$ In short, this 1968 study provides evidence against the burst nucleation model in the classic $(\mathrm{AgCl})_{n}$ formation system and under the specific conditions examined.

\subsection{Formation of colloidal silver halide in an open system: Sugimoto's classic system and efforts}

Sugimoto and co-workers ${ }^{99,100}$ developed a nucleation model for the formation of sparingly soluble, near monodisperse colloidal silver halide particles in an open system, in which the source of the monomer is introduced at a constant feeding rate (i.e., constant supply rate), $Q$. In one experimental system 
(a)

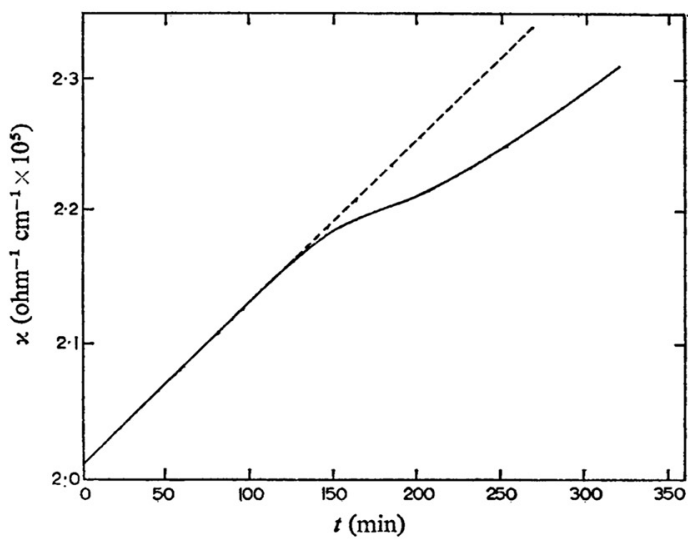

(b)

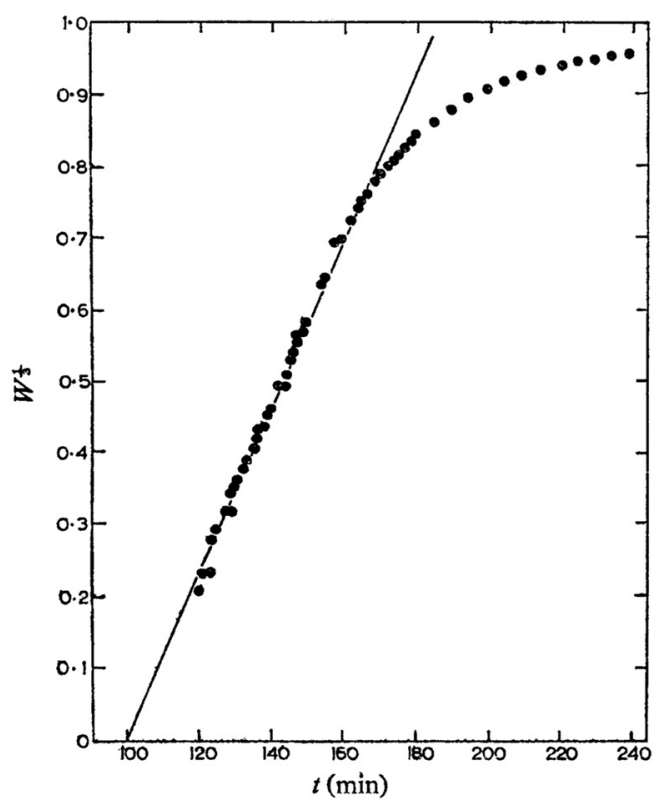

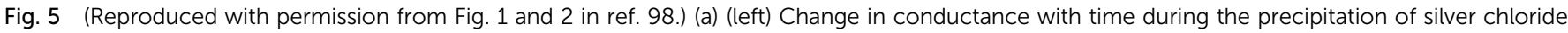

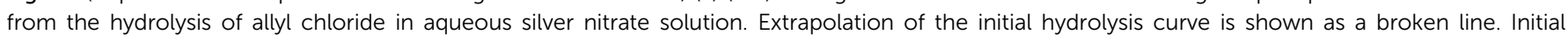

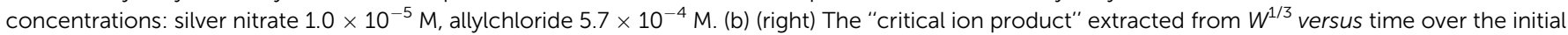

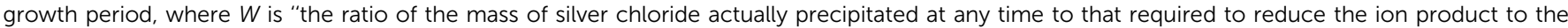
equilibrium solubility product $\left(1.77 \times 10^{-10}\right)$." 98 Reproduced with permission from ref. 98 . Copyright 1968 Royal Society of Chemistry.

carefully examined by Sugimoto and co-workers, ${ }^{100} \mathrm{AgNO}_{3}$ and $\mathrm{KBr}$ were separately introduced via "double jet" apparatus with two mixing tubes into a $\mathrm{pH} 5.0$ and $2 \mathrm{wt} \%$ gelatin solution (the latter to inhibit coagulation of AgBr particles) all with efficient, impeller mixing (see a schematic of the apparatus that is Fig. 2 in ref. 99). Samples were withdrawn at predetermined times followed by quenching at liquid $\mathrm{N}_{2}$ temperatures (or, as a control, adding a dye to arrest the growth or any dissolution of the particles); both procedures gave equivalent results in the subsequent TEM visualization experiments in this again welldesigned, carefully executed study. ${ }^{100}$ The final number of particles was used to infer the total number of "stable nuclei", $n_{+}^{\infty},{ }^{100}$ defined by the authors as the nuclei that can grow (versus, putatively, dissolve). ${ }^{100}$

Fig. 6a shows that the number of nuclei determined this way depends on the feeding rate of precursor as one might expect, while Fig. 6b shows that the particle number is a function of both temperature and time-contradicting the burst-nucleation model's assumptions $^{2}$ of a constant number of particles $v s$. time post burst nucleation. ${ }^{1}$ Fig. $6 \mathrm{c}$ shows the average radius $v s$. time of the growing particles (termed "nuclei" by the authors ${ }^{100}$ ). Relevant here is the minimum particle size observable by the microscopy employed was about $6 \mathrm{~nm}$, which we estimate corresponds to a $(\mathrm{AgBr})_{n}$ of $n \approx 2350$ - that is, a size presumably almost surely beyond any actual nuclei. Hence, more consistent is the alternative hypothesis that instead of "nuclei" the authors are actually monitoring the first observable cluster $(\mathrm{FOC})^{17}$ discernable by their particular microscopy technique.

Contrasting this $n$ value, stopped-flow kinetic measurements by Tanaka and Iwasaki (cited by Sugimoto in ref. 100 on p. 223) lead to much smaller $(\mathrm{AgBr})_{n}$ with $n$ values of just 3 to 10 as the implied nuclei. An educated guess is that the Tanaka and Iwasaki study is an early, perhaps seminal example of subsequent, but still rare, measurements of kinetically effective nuclei (KEN) ${ }^{17,26}$ of perhaps up to $\leq 10$ in this strong-bonding system. Additional studies reproducing and further analyzing this classic $(\mathrm{AgBr})_{n}$ system, combined with an appropriate mechanism or other model, would be most welcome and should in our opinion be a priority of the community interested in nucleation, growth and any subsequent agglomeration.

\subsection{Sugimoto's model: underlying key features and assumptions}

Anyone wanting to understand Sugimoto's important experiments and resultant model will need to read the original papers $^{99-102}$ carefully while striving to understand the model's key features, the assumptions behind the model, and where the Sugimoto model is similar to or different from the 1950 model or classical nucleation theory. Additionally, one needs to strive to understand where the mathematical equations given and the words used to describe the model jibe or seem disconnected, and the other interesting questions raised by the model, vide infra. Highly recommended additional reading is Chu, Owen, and Peters' concise derivation and analysis of the Sugimoto model, ${ }^{103}$ an important contribution that we will return to in a moment. The Sugimoto work merits careful examination as it makes the claim that the "LaMer diagram. . has actually been demonstrated by experiment". ${ }^{101}$ Hence, the classic, well designed and executed Sugimoto experiments are more generally believed to be one of the best pieces of evidence in support of the 1950 model. 


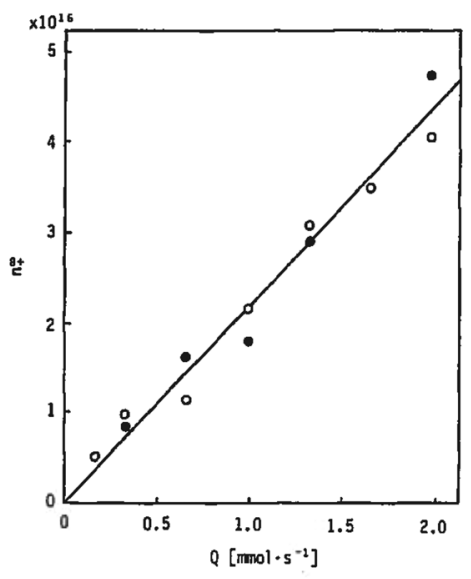

(b)

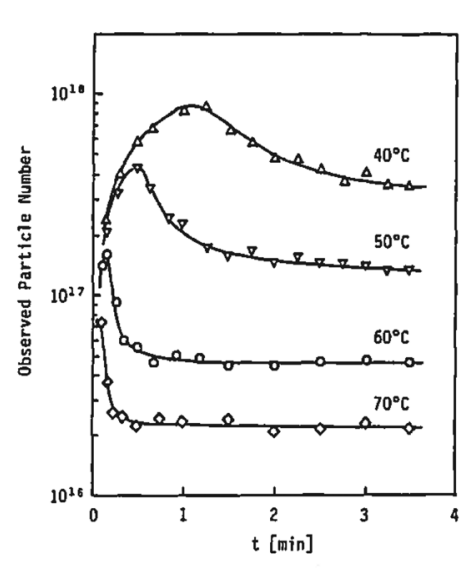

(a)

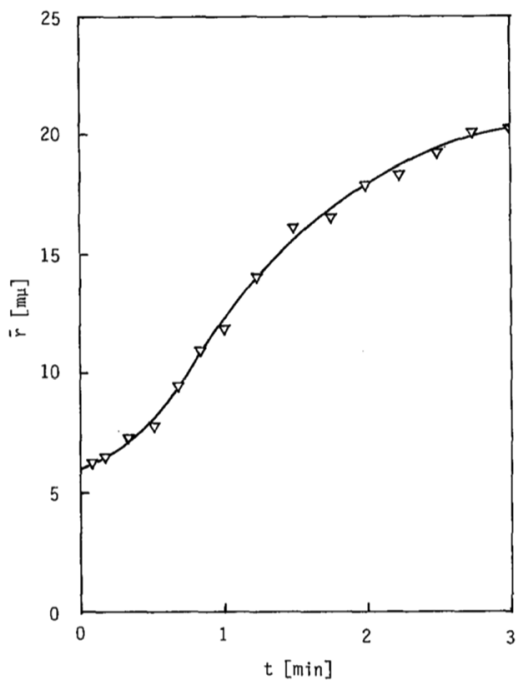

(c)

(b)

Fig. 6 (Fig. 9, 11 and 15 in ref. 100.) (a) The final number of observed particles $\left(n_{+}^{\infty}\right)$ as a function of $Q$, "the molal feed rate of the monomer source into the system", ${ }^{100}$ at $70{ }^{\circ} \mathrm{C}$ and $\mathrm{pBr} 3.0$ in the $(\mathrm{AgBr})_{n}$ formation open system. The closed circles represent a repeat set of experiments at the same $Q$ values. (b) The number of the observed particles $\left(n_{+}^{\infty}\right)$ as a function of time at different temperature $\left(Q=10^{-3} \mathrm{~mol} \mathrm{~s}^{-1}\right.$ and $\left.\mathrm{pBr} 3.0\right)$. (c) The change of the mean radius, $\bar{r}$, of "nuclei" (in the author's ${ }^{100}$ nomenclature; really the growing particle) as a function of time in the early stage of precipitations at $50{ }^{\circ} \mathrm{C}$. Observed lines are arbitrary and do not represent any fitting function. Reproduced with permission from ref. 100. Copyright 1992 Academic Press, Inc.

The basic equation of the Sugimoto model is eqn (3) below taken from his 2007 paper, ${ }^{99}$ an equation that breaks up the supply of solute $\left(Q V_{\mathrm{m}}\right)$ into its use to generate stable nuclei $\left(v_{+} \frac{\mathrm{d} n_{+}}{\mathrm{d} t}\right)$ or to grow those "stable" (i.e., growable) nuclei $\left(\dot{v}_{+} n_{+}\right):{ }^{99}$

$$
Q V_{\mathrm{m}}=v_{+} \frac{\mathrm{d} n_{+}}{\mathrm{d} t}+\dot{v}_{+} n_{+}
$$

In this equation (using seconds as the time unit, and where the quotes refer to the 2007 paper $\left.^{99}\right) Q$ is feed/supply rate in $\mathrm{mol} \mathrm{s}^{-1},{ }^{99}$ $V_{\mathrm{m}}$ is the "molar volume of the solid" in $\mathrm{cm}^{3} \mathrm{~mol}^{-1}$ (i.e., to convert the feed into a volume of solid per unit time, $\mathrm{cm}^{3} \mathrm{~s}^{-1}$ ), $v_{+}$is the "minimum particle volume of the stable nuclei" (again, the nuclei that by definition can grow), $n_{+}$is the "number of generated stable nuclei", $\frac{\mathrm{d} n_{+}}{\mathrm{d} t}$ is the rate of change of those stable nuclei, and $\dot{v}_{+}$is the "mean volume growth rate of the stable nuclei". Sugimoto's verbal description of his model from his 1992 paper in ref. 100 (with our explanatory comments provided in (parentheses)): "The theory is based on a nucleation model as origins of (i.e., as giving rise to) the product particles (that) are produced through a kind of Ostwald ripening from stationary unstable nuclei that are in a quasi-steady state of balance between the generation of their embryos by instantaneous reaction of the introduced reactants and either dissolution or growth". ${ }^{100}$ Note the proposed "instantaneous reaction" to generate the $n_{+}$nuclei embryos. This language will conflict with the diagram in Fig. 7 and the notion of nucleation occurring in some, albeit undefined, time period around $t_{\mathrm{m}}$ in Fig. 7 , in term causing confusion about if nucleation in the Sugimoto model is instantaneous or not.

The paper by Chu, Owen, and Peters provides an insightful rederivation and reanalysis of Sugimoto's model. ${ }^{103}$ Their rederivation starts as Sugimoto does with a pair of differential equations, eqn (4) and (5), that govern the concentration of solutes, $C(t)$, and populations of nuclei, $p_{\mathrm{T}}(t)$ respectively 


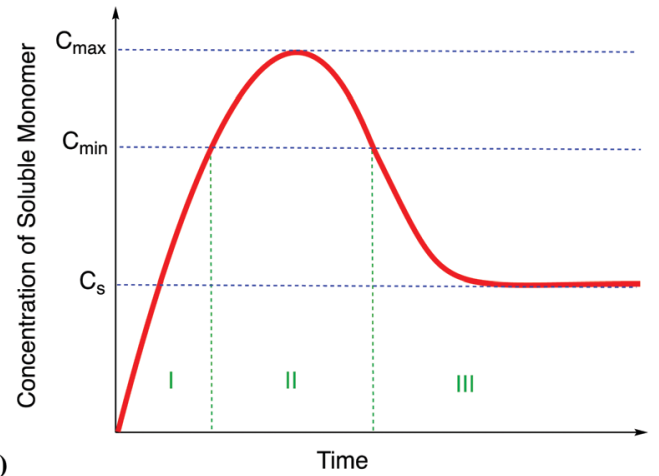

(a)

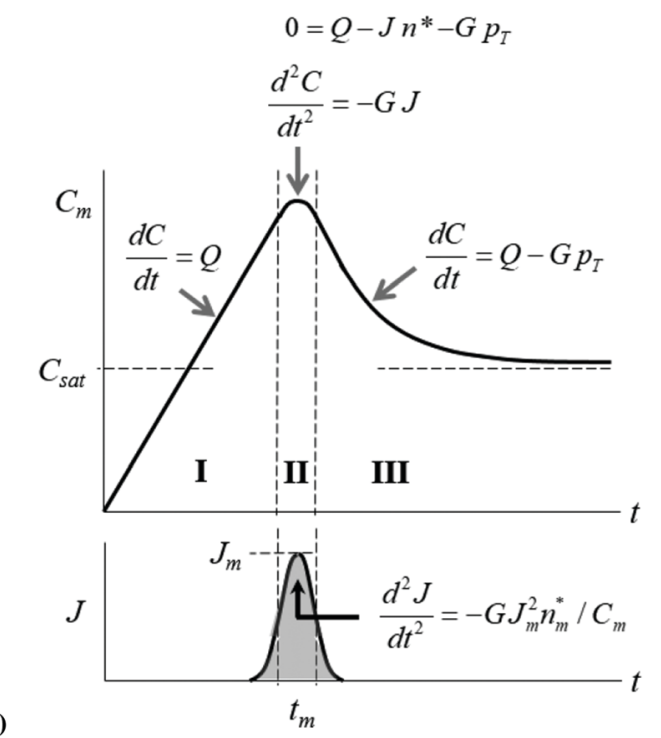

Fig. 7 (a) A schematic illustration of the 1950 hypothesis in picture form for the formation of colloidal particles, 1 originally for soluble monomer, $\mathrm{S}$, in the case of sulfur sol formation, $(\mathrm{S})_{n}$. (a) Is representative of what one finds in the literature, including what we have presented elsewhere, ${ }^{2}$ although LaMer's actual diagram ${ }^{2}$ in the original 1950 paper has a more linear initial rise. The hypothetical curve in (a) shows qualitatively the variation of soluble monomer concentration versus time during the whole formation process. $C_{s}$ is the saturation concentration (of the soluble monomer), $C_{\min }$ is the hypothetical minimum supersaturation for the nucleation, and $C_{\max }$ is the hypothetical limiting supersaturation, postulated stage I: formation of soluble monomer in solution. Postulated stage II: nucleation from the solution when the concentration of soluble monomer is higher than the critical supersaturation level $C_{\text {min }}$-as typically presented, albeit not shown as truly postulated "effectively infinite rate, burst nucleation". ${ }^{1}$ Postulated stage III: growth by diffusion when the supersaturation concentration is lower than $C_{\min }$, but higher than $C_{\mathrm{s}}$. It is critical to note that, in the intervening nearly 70 years, and despite the widespread reproduction and quoting of this figure, no such experimental time dependence of soluble sulfur monomer has ever been published, at least that we have been able to find from an exhaustive literature search. Reproduced with permission, including the figure caption, from ref. 2 . Copyright 2019 American Chemical Society. (b) The more quantitative version of the classic 1950 figure ${ }^{1}$ from B. Peters et al., that they describe by "each stage of a LaMer burst nucleation process is described by different equations that relate the solution concentration $C$, the nucleation rate $J$, the number of nuclei $p_{T}$, the growth rate $G$, and the nucleus size $n^{*} .103$ (b) Also more faithfully reproduces the initial linear rise given in LaMer's classic figure (Fig. 1 in the 1950 paper. ${ }^{1}$ ) Reproduced with permission from ref. 103. Copyright 2017 American Chemical Society. (the somewhat different symbols and nomenclature (i.e., vs. that used by Sugimoto) is retained below):

$$
\begin{gathered}
\frac{\mathrm{d} C}{\mathrm{~d} t}=Q-J n^{*}-G p_{\mathrm{T}} . \\
\frac{\mathrm{d} p_{\mathrm{T}}}{\mathrm{d} t}=J .
\end{gathered}
$$

where $n^{*}$ is the critical nucleus size, $J$ is the (constant; zeroorder) nucleation rate, $p_{\mathrm{T}}$ is the number of post-critical nuclei per volume, $G$ is the growth rate in solutes attached per time per nucleus, and $Q$ is the solute supply rate per volume. Combining eqn (4) and (5) following Sugimoto and under his assumptions of $\mathrm{d} C / \mathrm{d} t=0$ and $G=\mathrm{a}$ constant yields eqn (6), their version of Sugimoto's eqn (3) except not in volume-corrected units, plus eqn (7) for the number of nuclei as a function of time:

$$
\begin{gathered}
Q=\frac{\mathrm{d} p_{\mathrm{T}}}{\mathrm{d} t} n^{*}+G p_{\mathrm{T}} . \\
p_{\mathrm{T}}=\frac{Q}{G}\left(1-\exp ^{\left[-\left(t-t_{\mathrm{m}}\right) G / n^{*}\right]}\right) \text { for } t \geq t_{\mathrm{m}}
\end{gathered}
$$

From their treatment Peters et al. draw a LaMer-type diagram, Fig. $7 \mathrm{~b}$.

Professor Peters et al. then go on to make several important points: (i) eqn (6) "is true at and only at $t=t_{\mathrm{m}}$ ", where $t_{\mathrm{m}}$ corresponds to the time of the maximum supersaration, $C_{\mathrm{sat}}$, in the LaMer diagram, Fig. 7, vide infra (as that is the only place where the assumption of $\mathrm{d} C / \mathrm{d} t=0$ used to derive eqn (3) and (6) is true; see their Fig. 1 reproduced as Fig. $7 \mathrm{~b}$ that differs slightly from Fig. $7 \mathrm{a}$ in that Fig. $7 \mathrm{~b}$ tails asymptotically towards zero at longer times ${ }^{103}$ ). Peters $e t$ al. also note (ii) Sugimoto assumes, however, that his eqn (3) (and hence eqn (6)) remain valid for all $t \geq t_{\mathrm{m}}$; (iii) eqn (3) (and hence eqn (6)) are better viewed not as a differential equation, but as a condition on the solution of the coupled eqn (4) and (5); and (iv) that the initial condition invoked by Sugimoto of $p_{\mathrm{T}}\left(t_{\mathrm{m}}\right)=0$ is incompatible with any nucleation expression that depends monotonically on supersaturation, real nucleation beginning before $t_{\mathrm{m}}$. B. Peters et al. also note that $(\mathrm{v})$ the assumptions in the Sugimoto model of size- and concentration-independent growth are not physically correct, and they note that the Sugimoto model cannot account for narrow particle-size distributions (PSDs) because the sudden onset of nuclei then exponential decaying rate predicted by eqn (6) makes the predicted PSD "increase exponentially" up to an unrealistic maximum size of $G\left(t-t_{\mathrm{m}}\right)$. The Sugimoto model's many, non-realistic physical assumptions alone make questionable the (still creative) model's value, not unlike how the 9 assumptions underlying the original, 1950 burst-nucleation model make it inherently somewhat confusing and questionable ${ }^{2}$ from the beginning.

Reading the words Sugimoto uses to describe his model reveals the insidious disconnect that comes with physical models of dynamic chemical processes that are not described by balanced chemical reactions-the identical type of disconnect present with the burst-nucleation model $^{2}$ as discussed in detail in our Part I review and supported there by multiple citations of the relevant literature. ${ }^{2}$ Indeed, it takes some study, 
and the very helpful Peters et al. treatment cited above, to come to the following realizations about the model: (a) the original $n_{+}$ nuclei simply appear, in an ill-defined time window around $t_{\mathrm{m}}$ (i.e., no details are given as to how the $n_{+}$nuclei form, just that they are formed by some unknown pathway and mechanism approaching instantaneously). The model is, then, (b) a burst-nucleation model to form what are termed "stable" nuclei, $n_{+}$(i.e., defined as nuclei that are able to grow). For $\mathrm{Ag}^{+}$and $\mathrm{Br}^{-}$one might write this as: $(n-1)\left(\mathrm{Ag}^{+}+\mathrm{Br}^{-}\right) \rightarrow(\mathrm{AgBr})_{n-1}$ (they just show up, from supersaturated solution ${ }^{99,102,104}$ at an approaching infinite rate as the titles of two of Sugimoto's key papers confirm via the term "Spontaneous Nucleation...". 101,102) In this sense, then, an important point is that (c) the model is not really a "Theory of Nucleation..." as the title of his 1992 paper claims, ${ }^{100}$ no more than the 1950 model is a nucleation model. Both assume burst nucleation, even if one allows it to happen in some unspecified short time period centered around $t_{\mathrm{m}}$ in Sugimoto's model. Hence, both the Sugimoto and 1950 models are actually growth models.

Additionally in the Sugimoto model, in what is described only in words as an Ostwald ripening type of step, ${ }^{99-102}$ (i) unstable $(\mathrm{AgBr})_{n-1}$ can "dissolve" (e.g., by losing one or more $\mathrm{AgBr}$ ), but also (ii) grow to a "stable" nuclei, that we write as $(\mathrm{AgBr})_{n-1}+$ $\mathrm{AgBr} \rightarrow(\mathrm{AgBr})_{n}$, so that growth occurs by postulate only from "stable" nuclei of size $(\mathrm{AgBr})_{n}$ or larger, as CNT would have it. Nucleation and growth compete with one another for the precursor feed, $Q$, in the model and as eqn (3) teaches, what Sugimoto describes as "growth-limited nucleation". 101 Growth-limited nucleation will, however, always be present when nucleation and growth compete for a common precursor or other reagent, that is, are parallel reactions and, hence, compete kinetically for precursor. Hence and although "growth-limited nucleation" is not really a new or novel concept, an important part of Sugimoto's model is that it postulates nucleation and growth as parallel reactions (and not serial processes ${ }^{52}$ as comes out of some models claiming instantaneous nucleation ${ }^{52}$ but, interestingly and analogously are also really just growth models ${ }^{51}$ that have mathematically built in an assumed burst nucleation ${ }^{51}$ ).

The bottom line is that Sugimoto's model is really a growth model, one that has to date not been convincingly used for quantitative fitting of particle formation kinetics nor particlesize distribution data as is needed to give the growth model a compelling experimental test.

\subsection{Sugimoto's model for silver halide particle formation in a biphasic, closed systems}

The Sugimoto model ${ }^{100}$ for the formation of silver halides sols in open systems was modified for biphasic, closed systems. ${ }^{101,102}$ The first of two papers ${ }^{101}$ on AgX formation in closed systems reports the experimental examination of $(\mathrm{AgCl})_{n}$ formation in a Teflon-lined container with the following reagents ${ }^{99,101}$ (see p. 111 of ref. 99): $1.8 \times 10^{-2} \mathrm{M}$ (EtO) ${ }_{2} \mathrm{SO}_{2}$ (that is described as sparingly soluble in water ${ }^{101}$ ), $7.0 \times 10^{-3} \mathrm{M} \mathrm{KOH}, 2.0 \times 10^{-4} \mathrm{M} \mathrm{AgNO}_{3}, 2.0 \times$ $10^{-3} \mathrm{M} \mathrm{KCl}$, and $2.0 \mathrm{wt} \%$ gelatin. The implied series of reactions in this complex colloidal $(\mathrm{AgCl})_{n}$ formation system is not specified in a clear, equation form, but those reactions are necessary to understand the system and associated model. The reactions involved are presumably something reasonably close to what we have written below in an attempt to more clearly present and better understand this classic system:

$$
\begin{aligned}
(\mathrm{EtO})_{2} \mathrm{SO}_{2}+\mathrm{H}_{2} \mathrm{O} \rightarrow & (\text { EtO }) \mathrm{S}\left(\mathrm{O}_{2}\right) \mathrm{O}^{-} \mathrm{H}^{+}+\mathrm{EtOH} \text { (slow hydrolysis } \\
& \text { and } \mathrm{H}^{+} \text {formation) }
\end{aligned}
$$

$\mathrm{Ag}^{+}$-gelatin ${ }^{-}+\mathrm{H}^{+} \rightleftharpoons \mathrm{Ag}^{+}+$gelatin $^{-} \mathrm{H}^{+}\left(\mathrm{H}^{+}\right.$assisted release of $\mathrm{Ag}^{+}$)

$n\left(\mathrm{Ag}^{+}+\mathrm{Cl}^{-}\right)+$gelatin $^{-} \rightarrow(\mathrm{AgCl})_{n}$.gelatin ${ }^{-}$(fast, multi-step formation of gelatin-stabilized $(\mathrm{AgCl})_{n}$ colloid $)$

The gradual release of $\mathrm{Ag}^{+}$ion in this complex system was carefully monitored using a $\mathrm{Ag}_{2} \mathrm{~S}$-coated $\mathrm{Ag}$ electrode that, in turn, allowed comparison of the observed $\mathrm{Ag}^{+}$to that expected at equilibrium with $\mathrm{AgCl}$ according to the solubility product $K_{\text {sp }}=\left[\mathrm{Ag}^{+}\right]\left[\mathrm{Cl}^{-}\right]$. However, a critical point here is that the actual $K_{\text {sp }}$ used in the calculations is never given in the paper-despite the critical need for an independently determined $K_{\text {sp }}$ under the specific conditions of this complex system in order to place the studies on a firm foundation. (For reference, $K_{\mathrm{sp}}(\mathrm{AgBr})=7.7 \times$ $10^{-13}$ and $K_{\mathrm{sp}}(\mathrm{AgCl})=1.8 \times 10^{-10}$ at $25^{\circ} \mathrm{C}$ in pure water. ${ }^{105,106}$ ) Hence and unfortunately, the calculated maximum supersaturation ratio, $S_{\mathrm{m}}$, as given and used in the given in the paper (and in Sugimoto's nomenclature) $S_{\mathrm{m}}=\left[\mathrm{Ag}^{+}\right]_{m}\left[\mathrm{Cl}^{-}\right]_{\mathrm{o}} / K_{\mathrm{sp}}$ (where $S_{\mathrm{m}}=c a$. 3.5, Tables 2, 3 and Fig. 12 therein ${ }^{101}$ ) were not independently verified, may not be accurate, and hence remain as not-yet-reliable estimates (including the supersaturation ratio values, $S$, defined by Sugimoto and used in Fig. 8).

In what appears to be a widely cited, hence apparently generally believed, qualitative claim in the 2000 paper, ${ }^{101}$ the claim is made that the "LaMer diagram. . has actually been demonstrated by experiment". ${ }^{101}$ That is, the authors claim that their Fig. $9^{101}$ (reproduced as Fig. 8) supports and reproduces the famous, widely cited diagram in the 1950 paper. $^{1}$ If so, then Sugimoto's 1992 paper would be a landmark paper

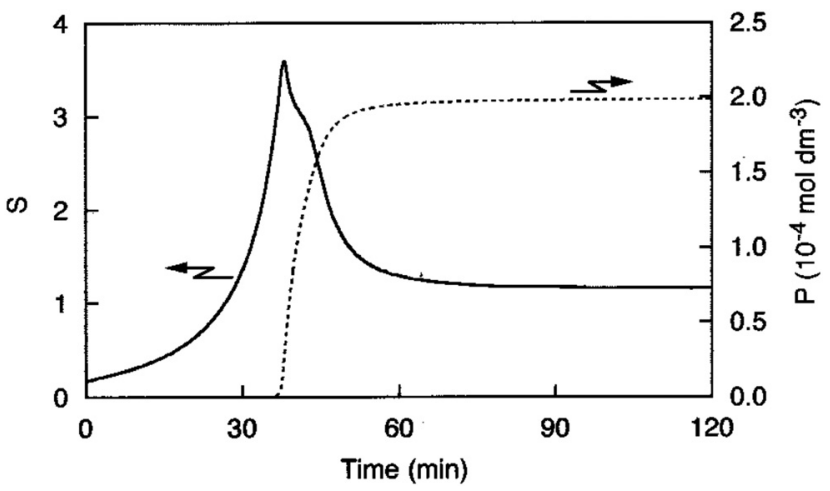

Fig. 8 (Fig. 9 from ref. 101.) The calculated supersaturation ratio, $S$, defined by Sugimoto and co-workers as $S=\left[\mathrm{Ag}^{+}\right]\left(\left[\mathrm{Cl}^{-}\right]_{0}-P\right) / K_{\mathrm{sp}}$ and shown on the left-most $y$ axis where $P$ (shown on the right-most $y$-axis) is

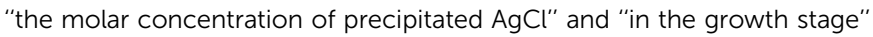
(as stated on p. 191 in ref. 101). The $\left[\mathrm{Ag}^{+}\right]$vs. time curve used as input into the $S$ equation to generate this Fig. 8 is given as Fig. 9 in ref. 101. Reproduced with permission from ref. 101. Copyright 2000 Elsevier. 
in supporting at least the qualitative picture and the often cited 1950 diagram from the 1950 paper. $^{1}$

Fig. 8 shows two experimentally determined phenomena: (i) the variation in the supersaturation ratio $v s$. time, $S=$ $\left[\mathrm{Ag}^{+}\right]\left(\left[\mathrm{Cl}^{-}\right]_{0}-P\right) / K_{\mathrm{sp}}$, as defined by Sugimoto (with a reminder of the caveat noted above, namely that the $K_{\mathrm{sp}}$ value used was not independently verified under Sugimoto's reaction conditions and apparatus), and Fig. 8 also shows (ii) $P$, the molar amount of $\mathrm{AgCl}$ precipitated out of solution vs. time expressed as a molar concentration. The results are interesting and a credit to Sugimoto and co-worker's experimental efforts, no matter what the best interpretation of the data turns out to be, as discussed next.

An examination of Fig. 8 reveals that a number of questions need to be addressed before Fig. 8 could be considered a compelling experimental recreation of the classic 1950 diagram and associated hypothesis about particle formation. (i) First, the y-axis in Sugimoto's Fig. 8 above is not just "monomer concentration" as in the 1950 model and classic figure (i.e., for this case soluble, $\left[\mathrm{Ag}^{+}\right]\left[\mathrm{Cl}^{-}\right]$), but $S$, the supersaturation ratio, so a ratio of concentrations; (ii) second, inspection of Fig. 8 in the 2000 paper $^{101}$ reveals that at $c a .40$ minutes $(\mathrm{AgCl})_{n}$ nanoparticles with a mean diameter of $3.3 \mathrm{~nm}$, and hence $n \approx$ 440 , have formed. This large $n$ value means that not just nucleation, but also considerable growth, has occurred by the 40 minute maximum in the supersaturation ratio, $S$, in Fig. 8. Indeed, Sugimoto defines the $P$ used in his $\left[\mathrm{Ag}^{+}\right]\left(\left[\mathrm{Cl}^{-}\right]_{0}-P\right)$ term in his $S=\left[\mathrm{Ag}^{+}\right]\left(\left[\mathrm{Cl}^{-}\right]_{0}-P\right) / K_{\mathrm{sp}}$ equation as $P$ being determined "in the growth stage". This would seem to strongly imply that the nucleation event(s) that the 1950 pictorial model strives to illustrate are likely mostly over before the experimental data near the $y$-maximum in Fig. 8 were collected-in turn suggesting the two are for fundamentally different net processes and not comparable. (iii) Third, it is hard to tell if the $\left[\mathrm{Ag}^{+}\right]\left(\left[\mathrm{Cl}^{-}\right]_{0}-P\right)$ quantity measured can give one the required concentrations, $\left[\mathrm{Ag}^{+}\right]\left[\mathrm{Cl}^{-}\right]$, because some of the reactions have slow kinetics (eqn (8) and perhaps (9)) including gelatin as a reagent-they might be providing the desired values, but it is hard to be sure; there is also the fact (iv) that the first part of the 1950 diagram's curve in Fig. 7a is concave, while the corresponding part in Fig. 8 is convex. A positive is (vi) that the critical placement of where the $C_{\mathrm{sat}}$ line goes on an experimental curve (i.e., $C_{\text {sat }}$ in Fig. $7 \mathrm{~b}$, or equivalently $C_{\mathrm{s}}$ in Fig. $7 \mathrm{a}$ ) is something that Sugimoto does achieve-his $S=1.0$ value is that line and if one uses the $S$ ratio.

The above important details so noted, the main problem at present with the claim that the "LaMer diagram...has actually been demonstrated by experiment" 101 is (v) that Fig. 8 may well be more consistent with other, non-LaMer models and mechanisms that show spikes in growth-note that no alternative explanations for Fig. 8 were proposed or explored. Later in Section 7.1 we will see that continuous nucleation and sizedependent growth can generate spiked concentration curves that (a) have some of the features of Sugimoto's intriguing Fig. 8, but are known to be (b) spiked growth curves-as Sugimoto's probably also is. Additionally, the peak maximum in the $\mathrm{Ag}^{+}$(and calculated concomitant start of the $\mathrm{AgCl}$ precipitation) in Fig. 8 may have more to do with the silver halide precipitation process, $m(\mathrm{AgCl})_{n}$.gelatin $^{-} \rightarrow(\mathrm{AgCl})_{n \cdot m}$ (ppt) $+m$ gelatin $^{-}$, which precedent says might be treatable kinetically as a bimolecularly nucleated, autocatalytic agglomeration process, ${ }^{5-58}$ an alternative hypothesis meriting experimental testing. The released gelatin ${ }^{-}$species could then bind previously freely diffusing $\mathrm{Ag}^{+}$, causing that value to drop past its maximum as seen in Fig. 8 .

More experimentation is needed to yield unequivocal conclusions regarding Sugimoto's interesting, valuable $\mathrm{AgCl}$ precipitation system, specifically: (i) a repeat of the experimental work, including the results reproduced in Fig. 8; (ii) determination of the applicable $K_{\mathrm{sp}}$ under the reaction conditions and then calculation of the true supersaturation with realistic error estimates, all with (iii) keen attention to Turnbull's work ${ }^{71}$ showing that variable, apparent supersaturation values are a problem due to heterogeneous nucleation probably in turn due to room dust, so that kinetics effects are creeping into one's attempts to measure reliable supersaturation values. ${ }^{71}$ Additionally, it will be critical to then (iv) test any and all possible models and mechanisms that might be able to fit quantitatively the resultant data analogous to Fig. 8. That needed research will likely give the researchers repeating Sugimoto's original 2000 work a keener appreciation for the cleverness and skills in the design and execution of that classic work. $^{101}$

A second paper by Sugimoto ${ }^{102}$ examines the formation of $(\mathrm{AgBr})_{n}$ colloidal nanoparticles using the same experimental system as in eqn (4)-(6) above for $\mathrm{AgCl}$ formation, but now with $\mathrm{KBr}$ so that the $\mathrm{Ag}^{+}$ions gradually released from the $\mathrm{Ag}^{+}$ -gelatin ${ }^{-}$complex are captured by $\mathrm{Br}^{-}$to yield colloidal $(\mathrm{AgBr})_{n}$, at least initially. The reported $K_{\mathrm{sp}}=1.77 \times 10^{-10}, 2.73 \times 10^{-10}$, $4.13 \times 10^{-10}$, and $6.15 \times 10^{-10} \mathrm{~mol}^{2} \mathrm{~L}^{-2}$ at $25,30,35$, and $40^{\circ} \mathrm{C}$, respectively, are used along with the electrochemically measured $\mathrm{Ag}^{+}$to provide curves for the supersaturation ratio, $S$, as a function of time in Fig. 15 of the 2000 paper $^{102}$ that are closely analogous in shape and features to, but not exactly identical to, Fig. 8, but now with a still-spiked maximum at 40 , 25,15 , and 10 minutes, respectively, which is again when the $\operatorname{AgX}(\mathrm{X}=\mathrm{Br})$ first is detected. The diameter of the $(\mathrm{AgBr})_{n}$ nanoparticles present at that time is $1.97 \mathrm{~nm}$, so that an average value of an again relatively large $n=83$ is present for the $(\mathrm{AgBr})_{n}$ when the $\mathrm{Ag}^{+}$peaks and the $\mathrm{AgBr}$ precipitation is treated as beginning. The final particle size is smaller by a factor of $1 / 6$, an effect attributed by the authors to the lower solubility of AgBr.

Once again, meriting future investigation here are efforts at (i) repeating this also classic work from Sugimoto's laboratories, (ii) verifying the $K_{\mathrm{sp}}$ employed, and (iii) finding mechanisms or other models that can quantitatively fit the sigmoidalshaped vs. time kinetics curves observed. Attention to the presence of dust and heterogeneous nucleation are two other issues that will need attention.

\subsection{Shiba and Okawa's study of silver bromide precipitation}

A 2005 paper by Shiba and Okawa ${ }^{107}$ reports a relationship between the supersaturation level, $S$, and the supply rate of 


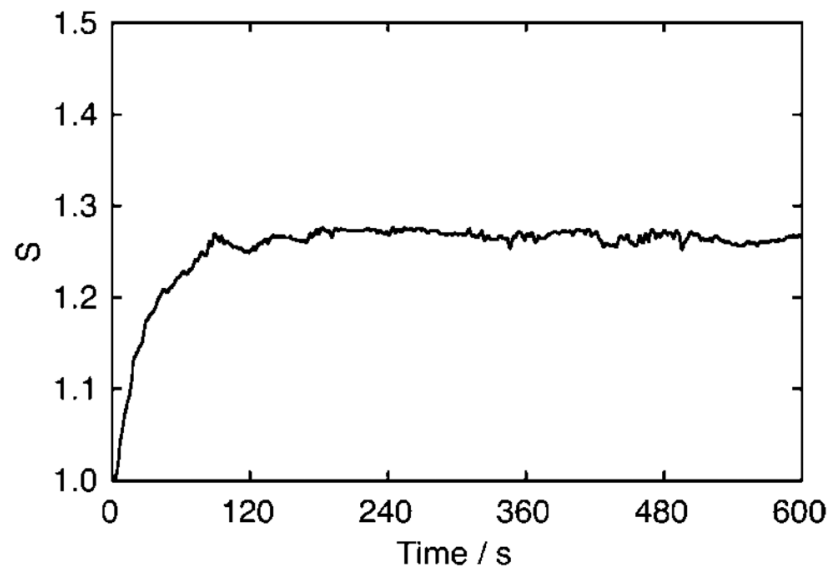

Fig. 9 (Fig. 1 from ref. 107.) Time evolution curve of the supersaturation, $S$, relative to saturation as determined from the $K_{\mathrm{sp}}$ for continuous feeding the reactant solutions of silver nitrate and potassium bromide to a seed solution of dispersed octahedral $\mathrm{AgBr}$ particles in $\mathrm{pBr} 2.0$ at $50{ }^{\circ} \mathrm{C}$. The supply rate of solute per particle was $Q / n r=2.56 \times 10^{-12} \mathrm{~mol} \mathrm{~s}{ }^{-1} \mathrm{~m}^{-1}$, where the mean radius of seed particles is $r=0.12 \mu \mathrm{m}$ and $n$ is the number of seed particles. Reproduced with permission from ref. 107. Copyright 2005 American Chemical Society.

solute for a related $\mathrm{AgBr}$ precipitation system. The authors measure $S$ as a function of time for the continuous feeding of the reactant solutions of silver nitrate and potassium bromide to a seeded solution of octahedral $(\mathrm{AgBr})_{n}$ particles, Fig. 9. The supersaturation in Fig. 9 slowly increases continuously to a steady-state value over nearly 100 minutes, with no appearance of any type of "burst" decrease, for example, as arguably expected given that $(\mathrm{AgBr})_{n}$ seeds were provided.

Overall, the AgX formation systems presented above provide a number of interesting results and are of fundamental value given that $\mathrm{Ag}^{+}$can be monitored to low levels electrochemically and because values for the $K_{\mathrm{sp}}$ of $\mathrm{AgX}$ are known in water. The pioneering Sugimoto and related systems merit further investigation and attempts to first reproduce the $S v s$. time curves and then quantitatively explain the resultant, reproducible curves with modern mechanisms or other models besides just the 1950 model.

\subsection{Formation of ternary silver halide nanocubes with mixed chloride and bromide ions}

A 2015 paper $^{108}$ reports the synthesis of photonic crystals of ternary silver chlorobromide $\left(\mathrm{AgCl}_{x} \mathrm{Br}_{1-x}, 0<x<1\right)$ with a highly uniform size, morphology, and crystallinity through the injection of $\mathrm{Ag}^{+}$ions into an ethylene glycol solution of $\mathrm{Cl}^{-}$and $\mathrm{Br}^{-}$ions containing poly(vinylpyrrolidone) for particle stabilization. The authors report that the size of the nanocubes is tunable by varying a number of parameters: (i) the molar ratio of $\mathrm{Cl}^{-}$to $\mathrm{Br}^{-}$ ions; (ii) the injection rate of $\mathrm{Ag}^{+}$ions; and (iii) the reaction temperature. Significantly, the formation of colloidal $\mathrm{AgCl}_{x} \mathrm{Br}_{1-x}$ nanocubes was monitored in real-time using in situ high-energy synchrotron X-ray diffraction. The results show that a fast injection rate of $\mathrm{Ag}^{+}$ions is critical for the formation of highly pure facecentered cubic crystalline $\mathrm{AgCl}_{x} \mathrm{Br}_{1-x}$ nanocubes, Fig. 10.

The authors claim the applicability of the 1950 model to their system and its resultant data, and interpret their data as

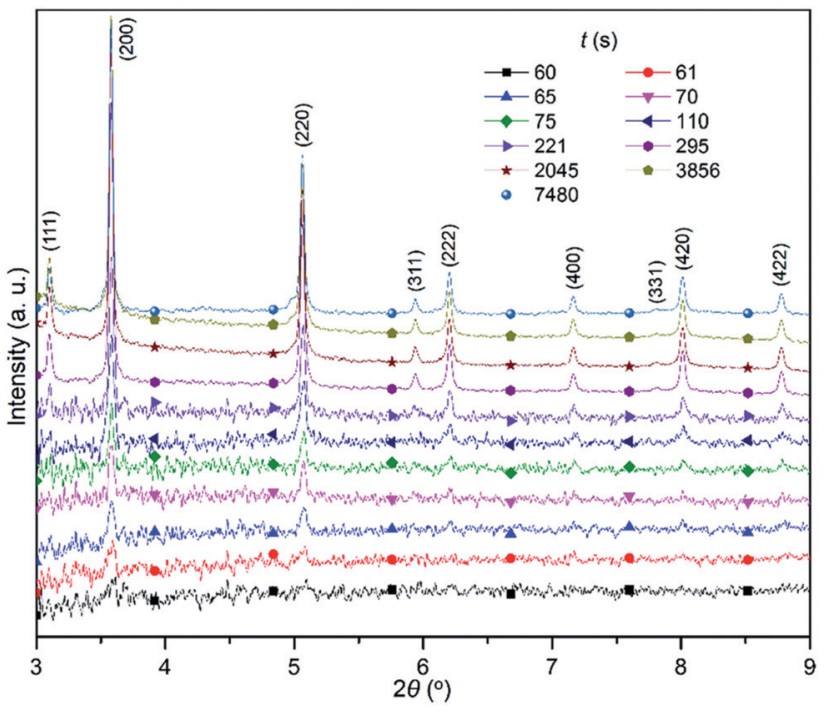

Fig. 10 (Fig. 3 from ref. 108). XRD patterns taken from the reaction mixture containing $\mathrm{KBr}$ and $\mathrm{NaCl}$ with a molar ratio $[\mathrm{KBr}] /([\mathrm{NaCl}]+[\mathrm{KBr}])=$ $4 / 8$ used for the synthesis of $\mathrm{AgCl}_{x} \mathrm{Br}_{1-x}$ nanocubes (SEM image in Fig. 1 from ref. 108). Different patterns were recorded at different times normalized against time when the $\mathrm{AgNO}_{3}$ solution was injected into the reaction mixture. Injected into the $12 \mathrm{~mL}$ ethylene glycol solution of $0.35 \mathrm{mmol} \mathrm{NaCl}$ and $0.70 \mathrm{mmol} \mathrm{KBr}$ plus $2.5 \mathrm{~g}$ polyvinylpyrolidone (PVP) was a $1.0 \mathrm{~mL}$ ethylene glycol solution of $\mathrm{AgNO}_{3}(0.34 \mathrm{M})$ at an injection rate of $1 \mathrm{~mL} \mathrm{~min}{ }^{-1}$ with the assistance of a syringe pump. The X-ray wavelength was $0.1771 \AA$. Reproduced with permission from ref. 108. Copyright 2015 Royal Society of Chemistry.

overall supporting "two sequential nucleation/growth processes. .." leading to a "bimodal size distribution" of $\mathrm{AgCl}_{x} \mathrm{Br}_{1-x}$ nanocubes. ${ }^{108}$ However, the authors did not consider a continuous nucleation model that (i) can explain the induction period and sigmoidal kinetic data one can estimate from the XRD patterns in Fig. 10 (Fig. 3 in that paper ${ }^{108}$ ) at a lower injection rate of $0.2 \mathrm{~mL} \mathrm{~min}^{-1}$ (Fig. S2 and S3, ESI $\dagger$ in that paper ${ }^{108}$ ); and (ii) that continuous nucleation is an arguably more natural explanation for the bimodal distribution for the nanocubes centered at 80 and $300 \mathrm{~nm}$ (Fig. 1a and Fig. S6a, ESI $\dagger$ in that paper ${ }^{108}$ ) -that is, two competing continuous nucleations rather than two burst nucleations of different compositions at difference times.

Hence, this $\mathrm{AgCl}_{x} \mathrm{Br}_{1-x}$ nanocubes system is another system where additional study and especially more kinetics and their mechanistic analysis would be welcome and might lead to definitive results about the underlying mechanism.

\subsection{Further silver halide studies and overall conclusions}

Table $\mathrm{S} 1$ in the EDI summarizes the studies discussed above as well as additional studies of AgX systems for the interested reader, including studies of reverse micellar systems. ${ }^{109,110}$ The most critical conclusions from the sum of studies of the $\mathrm{AgX}$ systems are that (i) these systems tend if anything to refute, rather than support, the concept of burst nucleation; (ii) Fig. 8, reproduced from Sugimoto's work, ${ }^{101}$ is a classic system that merits reinvestigation; (iii) Sugimoto's mathematical model has a number of non-realistic physical assumptions that anyone using that model needs to be aware of and take into 
account; and importantly (iv) there is no compelling support for burst nucleation that at least we could find in any of the silver halide particle-formation studies examined.

\section{Formation of semiconductor nanoparticles}

In the last three decades, semiconductor quantum dots have been the focus of a great deal of interest for optical and imaging applications ${ }^{111,112}$ and for solar energy harvesting, ${ }^{113,114}$ among other applications. ${ }^{115}$ Once again, understanding the mechanism of formation of, now, semiconductor nanocrystals is critical en route to reproducible, optimized syntheses of the highest-quality size and size-dispersion controlled semiconductor quantum dots. Because surface ligands are critical to semiconductor nanocrystals, we review first ligand-stabilized semiconductor quantum dots. Table S2 of the ESI $\dagger$ provides a detailed analysis of 26 papers that the reader especially interested in the next section, or in forming their own initial opinion about what follows, is urged to study before proceeding to the next section.

\subsection{Formation of semiconductor nanoparticles stabilized by surface ligands}

In the formation of semiconductor nanoparticles, the final particle size has been shown to be strongly dependent on the electrondonating, ligating ability of the stabilizing surface ligands. ${ }^{115}$ Few nanomaterial surfaces are bare, and ligands as well as solvents can adsorb, coordinate, and bond to nanomaterial surfaces. ${ }^{116}$ These "surface ligands" can, in turn, play important roles in nanoparticle synthesis to modulate nucleation, growth and agglomeration reaction kinetics and associated pathways. Surface ligands can regulate the accessibility and reactivity of the nanoparticle surface, ${ }^{117-119}$ for example by passivating otherwise coordinatively unsaturated surface atoms and stabilizing the resultant ligated nanoparticles while inhibiting flocculation. Stabilizing ligands are likely often critical to determining the size and size-distribution of the final nanoparticles. ${ }^{120}$

One of the main conclusions in the scholarly 2016 review of the works on indium phosphide nanoparticles by Cossairt, ${ }^{121}$ post a critical analysis of the semiconductor nanoparticle formation literature, is that the 1950 model is inapplicable to the formation of indium phosphide nanoparticles. The primary evidence for this conclusion is that the stabilizing ligands present on the surface of nanoparticles determines the size of the ultimate particles rather than the diffusion of monomeric species in solution, as the 1950 model postulates. ${ }^{1,121}$ Indeed, recent experiments by Cossairt and co-workers ${ }^{122}$ describe the formation of $(\mathrm{InP})_{n}$ quantum dots starting with a magic-sized nanocluster $\mathrm{In}_{37} \mathrm{P}_{20}\left(\mathrm{O}_{2} \mathrm{CCH}_{2} \mathrm{Ph}\right)_{51}$, which is shown to be a competent singlesource precursor for the formation of $(\mathrm{InP})_{n}$ nanocrystals.

In a study of colloidal indium phosphide by Peng and co-workers, ${ }^{123}$ the accessibility of active sites on the surface of InP and other III-V quantum dots is hypothesized to be the key for breaking the entanglement between nucleation and growth in this system. First, the authors found that the ligands around each nanocrystal are necessary to ensure their colloidal stability by studying the growth of nanoparticles on the pre-synthesized, small seeds of $(\mathrm{InP})_{n}$ quantum dots. Seeds of $(\mathrm{InP})_{n}$ nanocrystals were prepared by injecting a solution of $\left(\mathrm{Me}_{3} \mathrm{Si}\right)_{3} \mathrm{P}$ and octylamine in octadecene to a solution of indium(III) acetate and myristic acid in octadecene at $185{ }^{\circ} \mathrm{C}$ and then stirring the reaction mixture at $178{ }^{\circ} \mathrm{C}$ until the growth of InP nanocrystals to the desired size resulted. The reaction was monitored by UV-vis absorption along with TEM images taken after the reaction to determine the particle size distribution. Starting with the $(\mathrm{InP})_{m}$ seeds, the use of "surface-activation reagents" such as acetylacetone or acetic acid with additional starting materials (indium alkanoates and $\left.\left(\mathrm{Me}_{3} \mathrm{Si}\right)_{3} \mathrm{P}\right)$ yielded surface growth of the (InP) ${ }_{m}$ seed particles. The authors define a "surface-activation reagent" as a reactant that loosens the ligand shell, which the authors believe allows for the "complete separation of nucleation from growth". ${ }^{123}$ In the absence of surface-activation reagents, small particles were obtained implying the presence of new nucleation in addition to growth from the seed particles. Furthermore, surface-activation growth on seed particles was shown to be more broadly applicable to $(\mathrm{InAs})_{n}$ and III-V based core/shell quantum dots. ${ }^{123}$ The authors provide a schematic model, Fig. 11, whereby nanocrystal ligands ${ }^{124}$ can permit colloidal stability yet allow just growth under "surface activation" conditions.

Overall, the demonstration of surface ligand participation in the $(\mathrm{InP})_{n}$ system $^{123}$ is strong evidence indicating that nucleation is chemical-reaction-rate based and, hence, not "burst" in this $(\mathrm{InP})_{n}$ system. $^{123}$

Two very recent papers on (InP) $)_{n}$ nanocrystal quantum dot (QD) formation (i.e., recent enough that they are not included in Table S2 in the ESI $\dagger$ ) interpret their data in terms of continuous nucleation. ${ }^{125,126}$ Owen's system is a well-defined system with ${ }^{31} \mathrm{P}$ and UV-vis $(413 \mathrm{~nm})$ handles that merits a detailed kinetics and mechanistic study, although the slow, possibly rate-determining nature of the early precursor conversion step(s) may hinder insights into the desired nucleation, growth and any agglomeration steps present. ${ }^{125}$ Bawendi's paper cleverly compares continuous, interrupted, and variable rate injections of precursor as a way to yield larger particles with low (albeit unspecified) size dispersity. ${ }^{126}$ Continuous nucleation $^{3}$ and the "smaller grow faster than larger" ${ }^{\text {, } 61,62}$ are used to rationalize the results, although independent evidence for these concepts in the (InP $)_{n}$ system was not provided, ${ }^{126}$ not

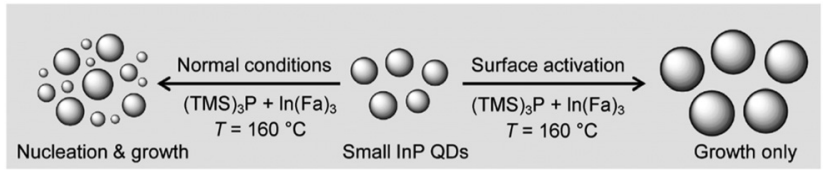

Fig. 11 (Fig. 1a in ref. 123). Two proposed reaction pathways for the growth of $(\operatorname{InP})_{n}$ quantum dots (QD) starting with the amine-based presynthesized seeds of InP QDs plus $\ln \left(\right.$ III) alkanoate, $\ln (\mathrm{Fa})_{3}$ (Fa = fatty acid carboxylate anion), and tris(trimethylsilyl)phosphine, (TMS) ${ }_{3} \mathrm{P}$, in the absence (left) or presence (right) of the surface-activation reagent acetylacetone. Reproduced with permission from ref. 123. Copyright 2017 Springer. 
unexpectedly as the work is focused on synthesis. A "persistent intermediate" is observed that is believed to be on the reaction pathway, although as discussed earlier pre- and post-steady state kinetics will be needed to support or refute this claim while distinguishing from off-path species. In short, these interesting systems are advancing our knowledge of (InP) particle synthesis so that kinetics and mechanistic studies, nanocrystals $(\sim 3.5 \mathrm{~nm}$ by TEM) is insensitive to the reaction conditions, including to the total concentration of the precursors and the initial cadmium to sulfur ratio, an observation that points towards the effect of carboxylate ligand-stabilization of the nanoparticles. That said, a goal for additional studies should be to determine the exact composition of the $\mathrm{Cd}_{a} \mathrm{~S}_{b}\left(\mathrm{O}_{2} \mathrm{CR}\right)_{c}$ nanocrystals under the various different conditions.

$$
\begin{aligned}
& 4 \mathrm{Cd}\left(\mathrm{O}_{2} \mathrm{CR}\right)_{2}+\mathrm{Cd}\left(\mathrm{S}_{2} \mathrm{PPh}_{2}\right)_{2} \rightarrow 4_{\mathrm{R}} \mathrm{N}_{\mathrm{O}} \mathrm{I}_{\mathrm{R}}^{\mathrm{O}}+\mathrm{Cd}\left(\mathrm{O}_{2} \mathrm{PPh}_{2}\right)_{2}+4(\mathrm{CdS})_{i} \\
& \mathrm{~m}(\mathrm{CdS})_{i}+\mathrm{n} \mathrm{Cd}\left(\mathrm{O}_{2} \mathrm{CR}\right)_{2} \longrightarrow(\mathrm{CdS})_{\mathrm{m}}\left(\mathrm{Cd}\left(\mathrm{O}_{2} \mathrm{CR}\right)_{2}\right)_{\mathrm{n}} \\
& \mathrm{R}=\mathrm{C}_{13} \mathrm{H}_{27}
\end{aligned}
$$

leading to additional insights into the detailed, underlying mechanisms, would be welcome for these important systems.

Peng and co-workers also report ${ }^{127}$ the synthesis of nearmonodisperse zinc blende $(\mathrm{CdSe})_{n}$ nanocrystals, coated with cadmium carboxylate ligands, starting with cadmium stearate and a selenium suspension in octadecene. Given that carboxylates are negatively charged, these seeds have excess $\mathrm{Cd}^{2+}$ ions on their surface and react with Se precursor in the presence of amine to form amine-terminated nanocrystals ${ }^{127}$ as illustrated schematically in Fig. 12. Addition of $\mathrm{Cd}\left(\mathrm{O}_{2} \mathrm{CR}\right)_{2}$, as shown in Fig. 12, gets one back to a species with surface $\mathrm{Cd}^{2+}$ ions so that the cyclic growth with added Se and amine can be repeated. The mean diameter of the resultant (CdSe) $)_{n}$ nanocrystals is found to increase linearly with the number of cycles as shown in Fig. 1e of that paper. ${ }^{127}$

The two papers by Peng and co-workers ${ }^{123,127}$ discussed above demonstrate the role of surface ligands in the formation as well as in the stabilization of semiconductor quantum dots. A similar ligand effect has also been observed in the case of coinage metal chalcogenide nanocrystals obtained from N-heterocyclic carbene (NHC) synthons, ${ }^{128}$ in which the size of metal chalcogenide particles depends on the electron donating ability of the NHC ligands on the surface of nanoparticles.

In contrast to a 2014 paper $^{129}$ claiming the synthesis of bare $(\mathrm{CdS})_{n}$ nanocrystals from the thermal decomposition of cadmium(II) stearate and sulfur at $260{ }^{\circ} \mathrm{C}$, Owen and co-workers ${ }^{130}$ reported the formation of cadmium sulfide nanocrystals with tetradecanoate surface termination from the heating of $\mathrm{Cd}\left(\mathrm{S}_{2} \mathrm{PPh}_{2}\right)_{2}$ and cadmium tetradecanoate ( $\geq 4$ equiv.) to $240{ }^{\circ} \mathrm{C}$ with the complete conversion of $\mathrm{Cd}\left(\mathrm{S}_{2} \mathrm{PPh}_{2}\right)_{2}$ to the nanoclusters, as shown in eqn (11). Interestingly, the final average size of $(\mathrm{CdS})_{n}$
The nanoparticle formation reaction was monitored by ${ }^{31} \mathrm{P}$ NMR, UV-vis, and FT-IR spectroscopies. Fig. 13 shows the formation of $(\mathrm{CdS})_{n}$ nanocrystals versus time, with the caveat that the temperature ramp complicates the early-time kinetics (especially the first 5 minutes) so that, for example, an unequivocal determination of whether or not an induction period is present was not possible. Owen and co-workers were able to determine that "nucleation has a first order dependence on total precursor concentration". ${ }^{130}$ Additional work on the early-time kinetics of this interesting, well-defined system, ideally under isothermal kinetics conditions if possible, would be a welcome addition to the literature.

\subsection{Formation of semiconductor nanoparticles by the "Heating-Up" method}

A 2012 paper by Kim and Jang ${ }^{131}$ reports the synthesis of $(\mathrm{CdSe})_{n}$ nanocrystals with tunable morphology and optical properties via a microwave-assisted polyol process. The CdSe nucleation and growth kinetics along with the choice of precursor $\left(\mathrm{CdCl}_{2}\right.$ or $\left.\mathrm{CdO}\right)$ and solvent (ethylene glycol or glycerol) determine the resulting nanocrystal size and shape. $\mathrm{XRD}$ and TEM were used to characterize the $(\mathrm{CdSe})_{n}$ nanocrystals. However, no kinetics data monitoring the time dependence on either particle size or precursor/product concentration were reported. The authors interpret the anisotropic growth of the (CdSe $)_{n}$ nanocrystals using the 1950 model in a qualitative discussion and using two different curves (Fig. 8c of that paper ${ }^{131}$ ), one with a short time-span in the nucleation stage for the low solubility $\mathrm{CdO}$ precursor and one with a long time-span in the nucleation stage for $\mathrm{CdCl}_{2}$, which is highly

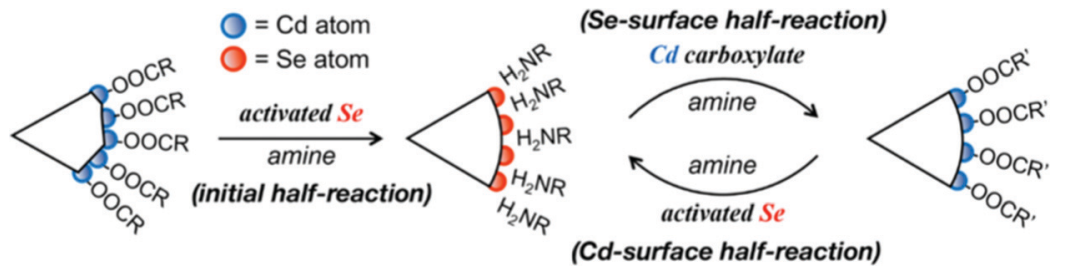

Fig. 12 (Fig. 1a in ref. 127) Scheme illustrating growth starting on CdSe quantum dots (QD) seeds via synthesis cycles that start with cadmium stearate and a selenium suspension in octadecene plus fatty amine for selenium activation. The initial half-reaction with the amine-activated selenium yielding amine-terminated nanocrystals. The second half-reaction adds cadmium carboxylate to yield the carboxylate terminated nanocrystals. Additional cycles yield additional growth. Reproduced with permission from ref. 127. Copyright 2018 American Chemical Society. 

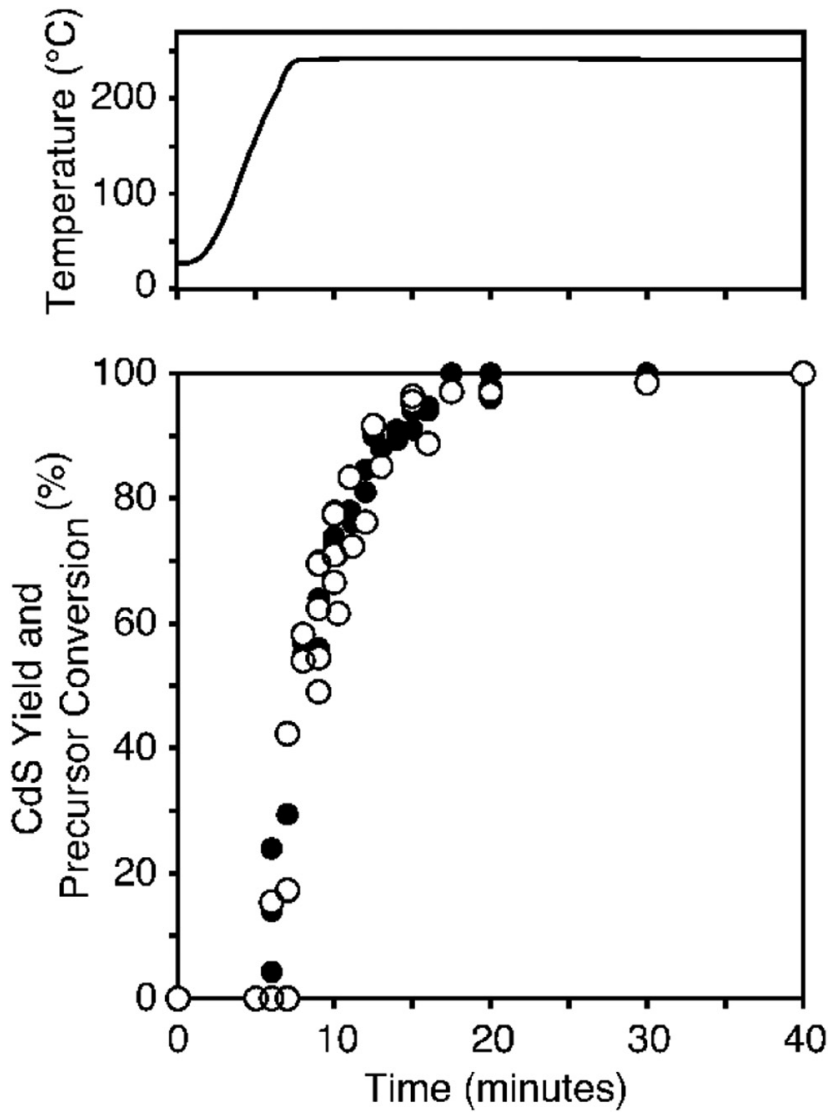

Fig. 13 (Fig. 7 of ref. 130). Kinetics data showing both precursor conversion (filled circles) and nanocrystal formation (empty circles), determined by ${ }^{31} \mathrm{P}$ NMR and UV-vis absorption spectroscopies, respectively. The data points, and thus two reaction rates, correspond closely with one another. The top panel shows the temperature ramp during a typical reaction. Reproduced with permission from ref. 130. Copyright 2012 American Chemical Society.

soluble in ethylene glycol. Despite their citation of the 1950 model, the authors do not have definitive, quantitative evidence to support any mechanism underlying the formation of $(\mathrm{CdSe})_{n}$ nanocrystals under their reaction conditions. Direct, short-time-resolved kinetics studies of semiconductor nanoparticle formation by ideally multiple in operando physical methods are needed and should lead to better understanding of the mechanism(s) of formation of such as $(\mathrm{CdSe})_{n}$ semiconductor nanoparticles.

In this regard, a state-of-the-art 2015 in situ SAXS and WAXS study is noteworthy in both what it achieves, as well as what remains to be done to elucidate additional kinetics and mechanistic insight. ${ }^{132}$ The precursors in eqn (12) were studied by the heating-up method, starting at $150{ }^{\circ} \mathrm{C}$ and heating to $240{ }^{\circ} \mathrm{C}$ at $1{ }^{\circ} \mathrm{C}$ per minute. The balanced reaction was not given, and the source of electrons to reduce the Se to $\mathrm{Se}^{2-}$ are not known, but the reaction as far as one can write it is shown in eqn (12):

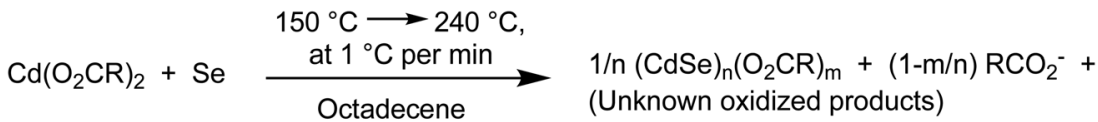

$$
\begin{aligned}
& \left(\mathrm{R}=\mathrm{CH}_{3}\left(\mathrm{CH}_{2}\right)_{12} \mathrm{CO}_{2}^{-}=\text {myristate }\right)
\end{aligned}
$$

The most important results in the study are that direct, in situ SAXS and WAXS reveals an initial lamellar phase, melting to a micellar pre-nucleation phase, then the firstobservable clusters (FOCs ${ }^{17}$ ) of size $\geq 1 \mathrm{~nm}$ and hence size ${ }^{133}$ $(\mathrm{CdSe})_{x}, x \geq 10$, at $218{ }^{\circ} \mathrm{C}$, plus subsequent growth by an unknown mechanism. The study was also able to rule out diffusion-controlled growth by showing that the observed growth phase is $10^{8}$ slower than diffusion control. The reduction of Se to $\mathrm{Se}^{2-}$ is identified as the slow step of the reaction, as one might expect and consistent with other studies cited in the paper. The authors rationalize their work in terms of CNT and the 1950 model, stating that "Overall, a LaMer-type mechanism is observed", a statement inconsistent with their own disproof of diffusion-controlled growth that is an integral part of the 1950 model. Hence, still needed to uncover the true underlying mechanism are: (i) establishment of the balanced reaction and especially the source of the reductant for the $\mathrm{Se}$ to $\mathrm{Se}^{2-}$ reduction that looks to be the rate-determining step, (ii) development of either a faster reductant that, then, may not obscure the desired nucleation and growth kinetics, or development of a way to study the reaction isothermally while starting at a higher temperature close to $218{ }^{\circ} \mathrm{C}$, and then (iii) kinetics and a rate law under those isothermal conditions in order to identify the true underlying mechanism.

\subsection{Formation of semiconductor nanoparticles by the hot-injection method}

A 2013 paper by Mukai and co-workers ${ }^{134}$ reports the synthesis of colloidal $(\mathrm{PbS})_{n}$ quantum dots by hot-injection of an oleylamine and sulfur solution into a suspension of $\mathrm{PbCl}_{2}$ in oleylamine at 70 or $80{ }^{\circ} \mathrm{C}$. The resulting $(\mathrm{PbS})_{n}$ nanocrystals are unfortunately not well characterized. Additionally, the existence of potential surface ligands was not investigated. This system is similar to the $(\mathrm{CdSe})_{n}$ nanocluster system described in Section 4.1, where oleylamine surface ligands are present. Hence, it is likely the surface of the PbS nanocrystals are aminepassivated, a point that merits additional study.

The authors claim that the nucleation and growth of the $(\mathrm{PbS})_{n}$ nanocrystals occur by the 1950 model, but this claim is presented without any kinetics data to support or refute this claim. The authors claim the length (time period) of the nucleation period is dependent on the degree of supersaturation, but, again any type of quantitative analysis as a function of any true, measured supersaturation is missing. In short, the true mechanism underlying $(\mathrm{PbS})_{n}$ nanoparticle formation remains to be established, ideally after first establishing the average $\left(\mathrm{Pb}_{a} \mathrm{~S}_{c} \text { Amine }_{c}\right)_{n}$ composition of the particles and then ideally by in operando monitoring of the kinetics of particle formation by $\geq 2$ direct physical methods. 


\subsection{Additional papers on the formation of semiconductor nanoparticles and the 1950 model}

Detailed summaries of the 26 papers citing the 1950 model and reporting the formation of semiconductor nanoparticles can be found in Table $\mathrm{S} 2$ in the ESI. $\dagger$ In addition to the papers discussed above and added ${ }^{140,141}$ to this section, additional papers on the following systems are provided in Table S2 (ESI $\dagger$ ): lead sulfide, ${ }^{135}$ cadmium sulfide, ${ }^{136-138}$ cadmium selenide ${ }^{139,140}$ indium phosphide, ${ }^{141}$ zinc sulfide, ${ }^{142}$ silver sulfide, ${ }^{143}$ fullerene nanowires, ${ }^{144}$ hot-injection, ${ }^{145,146}$ and continuous microwave-assisted gas-liquid segmented flow. ${ }^{147}$

The bottom line is that direct, compelling evidence for burst nucleation and diffusion-controlled growth in semiconductor nanoparticle formation is missing, a surprising insight given the level of at least apparent acceptance of the 1950 model in the area. Additional, detailed kinetics and mechanistic studies are needed probing especially the nucleation step(s), but also the growth step(s), of semiconductor particle formation systems. Several systems ripe for such studies were identified.

\section{Formation of metal nanoparticles}

The literature of metal nanoparticle formation is vast from investigations over at least three decades. A search of nanoparticle formation in Web of Science reports 18700 citations since 1990 and 9200 in just the past 5 years up to January 2019. (A search of "metal nanoparticle formation" yields 4050 hits, while a search of "gold nanoparticle formation" gives 4100 hits and, as an example, "silver nanoparticle formation" yields 2700 hits. Hence, the search term "metal nanoparticle formation" alone does not pick up all different metals.) Due to the sheer volume of research conducted on transition- and other metal-nanoparticle formation, this section will be focused on selected, especially highly investigated metals (gold, iridium, platinum, silver, and palladium) that have contributed significantly to the development of physical models or mechanisms for nucleation and growth, with of course an eye to those that cite or bear on the 1950 particle formation model. A full list of 69 references to metal nanoparticle formation related to the 1950 model can be found in Table S3 in the ESI, $\dagger$ covering the specific metals: gold, ${ }^{148-153}$ iridium, ${ }^{3,17}$ platinum, ${ }^{154,155}$ silver, ${ }^{156-158}$ palladium, ${ }^{159-161}$ and indium. ${ }^{162}$ Once again we strongly recommend prereading and studying the entries in Table S3 (ESI $\dagger$ ) before proceeding, as the data and evidence summarized there will allow the reader to make up their own opinions before reading the next section. That said, the following section is the one where the PI and his students have the greatest amount of published expertise.

\subsection{Formation of gold colloids via the Turkevich method}

The formation of homogenous gold systems dates to 1857 with Faraday's research on gold colloids. ${ }^{163}$ As mentioned back in Section 1.6.1, the best-known early research on gold nanoparticles is Turkevich's 1951 classic citrate reduction-based synthesis of gold nanoparticles/gold sols, ${ }^{5}$ influential work that was often ahead of its time in the understanding and insights it provides. In recent years, several other groups have performed additional notable investigations of gold nanoparticle formation using a variety of techniques: $\mathrm{pH}$ variation, ${ }^{164} \mathrm{UV}$-Visible spectroscopy, ${ }^{165-168}$ and X-ray based techniques. ${ }^{169-172}$

5.1.1. Turkevich's classic gold nanoparticles and relevance to the 1950 model. Just a year after publication of the 1950 model, ${ }^{1}$ John Turkevich published his first paper describing the synthesis of spherical colloidal gold particles ${ }^{5}$ of $20.0 \pm$ $1.5 \mathrm{~nm}( \pm 7.5 \%$ dispersion) from the reduction of dilute auric acid by sodium citrate. ${ }^{6}$ Turkevich and co-workers performed kinetics and particle-size measurements using several techniques: slit-ultramicroscopic examination to monitor the rate of gold nuclei formation; nephelometry to monitor gold particle formation with better reproducibility and accuracy than the slit-ultramicroscopic method; and electron microscopy to determine the particle formation vs. time curve along with the particle-size distribution.

Fig. 14 shows the concentration of trivalent gold ions as a function of time during the gold sol formation from a sodium citrate and chloroaurate mixture. The data indicate a sigmoidal consumption curve as the gold is reduced (see Fig. S1 in the ESI $\dagger$ for the original data). This sigmoidal kinetics curve is characteristic of slow, continuous ${ }^{3}$ (not "burst") nucleation and autocatalytic (not "diffusion-controlled") growth. Indeed, the fit of the kinetics data in Fig. 14 to a simple 2-step

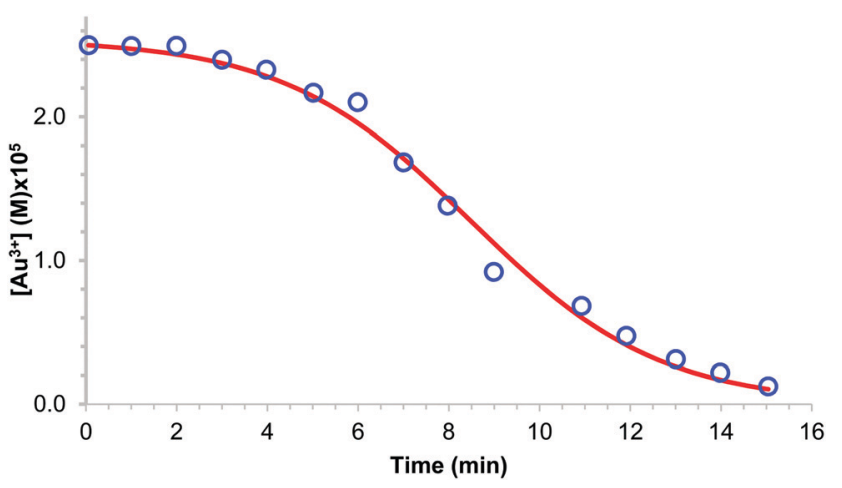

Fig. 14 Plot of $\mathrm{Au}(\mathrm{III})$ ion concentration versus time during the gold-sol formation from a sodium citrate and chloroaurate mixture measured by withdrawing aliquot portions at prechosen times, placing them into a concentrated potassium iodide solution to quench the reaction, titrating the $\mathrm{I}_{2}$ formed iodine with an excess of standard thiosulfate solution, and then titrating the remaining thiosulfate with a standard iodine solution. The blue circles represents the experimental data obtained by digitizing the scanned plot in Fig. 11 on page 65 of the 1951 paper, ${ }^{5}$ an original plot reproduced as Fig. S1 of the ESI† accompanying this paper for the convenience of the reader. The red solid curve shows the fit of data to a 2-step mechanism ${ }^{3}$ of $\mathrm{A} \rightarrow \mathrm{B}$ (rate constant, $k_{1}$ ) and then autocatalytic surface-growth, $\mathrm{A}+\mathrm{B} \rightarrow 2 \mathrm{~B}$ (rate constant $k_{2}$ ), yielding the rate constants for continuous nucleation of $k_{1}=(7.9 \pm 1.6) \times 10^{-3} \mathrm{~min}^{-1}$ and for chemical-reaction-controlled, autocatalytic surface growth of $k_{2}=0.19 \pm$ $0.01 \mathrm{M}^{-1} \mathrm{~min}^{-1}$, with $R^{2}=0.9923$ and reduced $\mathrm{Chi}$-Sqr $=6.78 \times 10^{-3}$, and where $A$ is the $A u(I 1)$ starting material, and $B$ is by definition ${ }^{3}$ the average, growing $\mathrm{Au}(0)_{n}$ particle. The choice of the 2-step mechanism to fit the kinetics is because doing so allows a ready demonstration that nucleation is not burst nor is growth diffusion controlled while using a normal, easily understood, classical kinetics treatment of the data that yields well-defined rate constants for the balancedequation-defined continuous nucleation and autocatalytic surface-growth steps. 
Table 3 The rate constants $k_{1}$ for the slow, continuous nucleation and $k_{2}$ for autocatalytic surface growth during the formation of $A u(0)_{n}$ nanoparticles obtained from a sodium citrate reduction of chloroaurate at the four temperatures shown

\begin{tabular}{lllr}
\hline$T\left({ }^{\circ} \mathrm{C}\right)$ & $k_{1}, \mathrm{~min}^{-1}$ & $k_{2}$, ("particles per volume") ${ }^{-1} \mathrm{~min}^{-1}$ & $R^{2}$ \\
\hline 15.4 & - & - & - \\
30.0 & $(8.0 \pm 1.9) \times 10^{-4}$ & $(1.59 \pm 0.08) \times 10^{1}$ & 0.99698 \\
39.0 & $(7.0 \pm 3.9) \times 10^{-4}$ & $(3.84 \pm 0.27) \times 10^{1}$ & 0.99516 \\
49.5 & $(2.0 \pm 0.7) \times 10^{-3}$ & $(6.68 \pm 0.39) \times 10^{1}$ & 0.99704
\end{tabular}

mechanism $^{3}$ consisting of slow continuous nucleation and nondiffusion-limited, autocatalytic surface growth demonstrates definitively that neither burst nucleation nor diffusion-controlled growth are part of Turkevich's classic system. The autocatalytic growth rate constant from the curve-fit in Fig. 14, $k_{2}=0.19 \pm$ $0.01 \mathrm{M}^{-1} \min ^{-1}$, is roughly $\sim 10^{12}$ times slower than diffusion controlled and if one uses $\sim 10^{12} \mathrm{M}^{-1} \min ^{-1}$ for a diffusioncontrolled reaction of a small molecule or small nanoparticle nucleus. Diffusion control for larger particles will of course be smaller than $\sim 10^{12} \mathrm{M}^{-1} \mathrm{~min}^{-1}$, but not more than an order of magnitude or two, so that $k_{2}=0.19 \pm 0.01 \mathrm{M}^{-1} \mathrm{~min}^{-1}$ is still at least $\sim 10^{10}$ times slower than diffusion control in this specific system.

In 1951 Turkevich provided the particle number versus time, and then particle number versus temperature, data reproduced in Fig. $15,{ }^{5}$ data that are the first, compelling, classic

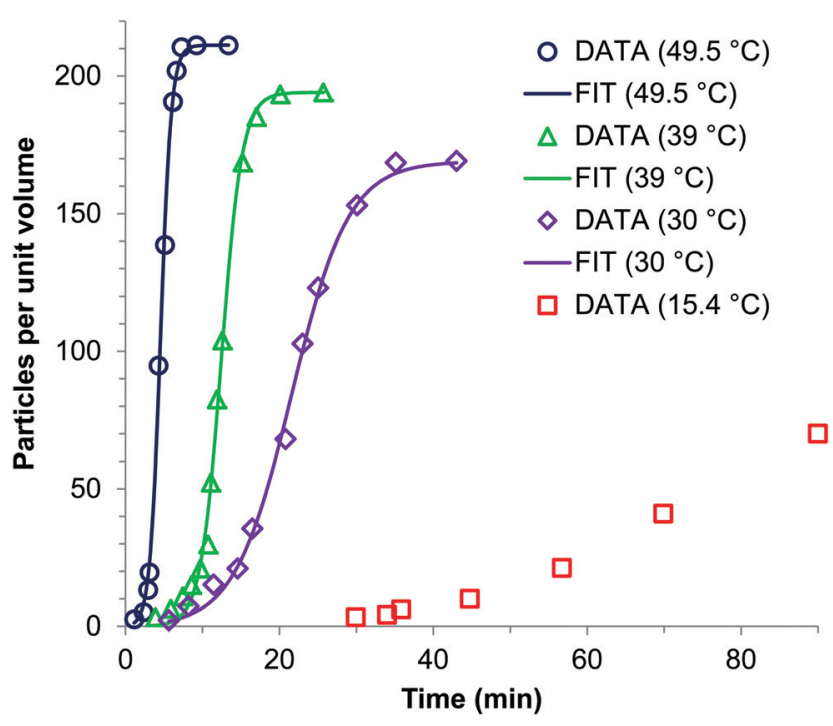

Fig. 15 Plots of the number of gold $(0)$ nanoparticles per unit volume versus time during the gold sol formation from a mixture of sodium citrate and chloroaurate at various temperatures (red squares at $15.4{ }^{\circ} \mathrm{C}$, purple diamonds at $30.0{ }^{\circ} \mathrm{C}$, green triangles at $39.0{ }^{\circ} \mathrm{C}$, and dark blue circles at $\left.49.5^{\circ} \mathrm{C}\right) .{ }^{5}$ The data were obtained via nephelometry. The solid curves show the fits of data to a 2 -step mechanism ${ }^{3}$ yielding the rate constants given in Table 1. (The data at $15.4{ }^{\circ} \mathrm{C}$ cannot fit because the final, maximum number of particles is missing.) The shortening of the induction period of each curve as temperature increases-that is, the increase in the nucleation rate constant $k_{1}$, as shown in Table 2, is prima facie evidence for the dependence of nucleation on temperature-an experimental fact inconsistent with the assumption of temperature independence in the 1950 model (see the Part I review ${ }^{2}$ for details of the 9 approximations/assumptions underlying the burst-nucleation model). Fig. S2 in the ESI $\uparrow$ presents the original data for the convenience of the reader. evidence disproving the 1950 model as it might be applied to his $\mathrm{Au}(0)_{n}$ formation system. Those data show the particle numbers versus time are (i) not instantaneous, (ii) not constant, nor (iii) temperature independent. Turkevich also noted ${ }^{5}$ that fluctuation theory (as underlies the burst-nucleation model, as discussed back in Section 1.3) could not explain "the marked temperature dependence of nucleation from the fluctuation point of view". 5,173,174 More specifically, Turkevich argued his "investigation indicates that the nuclei are $\sim 30 \AA$ diameter and this would involve a fluctuation of the order of a million of gold atoms." 5 Turkevich reached these numbers because his largest final $\mathrm{Au}(0)_{n}$ particle size was $240 \AA$ (i.e., $24.0 \mathrm{~nm}$ ) and hence $c a$. half a million gold atoms, $\mathrm{Au}_{\sim 428000}$. Turkevich was making the point that this early theory implied statistical fluctuations of apparently nearly all those millions of atoms in order to hypothetically yield putatively instantaneously formed nuclei, all $c a$. Au $\sim 840$ in size, something his comment indicates that he considered an extremely improbable event. For the record, Turkevich's observed "nucleus" $\operatorname{size}^{5}$ of $\sim 3 \mathrm{~nm}$ equals $\sim 840 \mathrm{Au}$ atoms, $\mathrm{Au} \sim 840$ (what we would now say was actually the first observable cluster (FOC) ${ }^{17}$ by the methods available at the time). The kinetically effective nucleus in the Turkevich $\mathrm{Au}(0)_{n}$ system is still not known to our knowledge, but an educated $^{17,26,27}$ guess is that the KEN is likely much smaller than $\mathrm{Au}_{\sim 840}$, and perhaps something closer to $\mathrm{Au}_{2-3}$.

The resultant rate constants $k_{1}$ and $k_{2}$, as a function of temperature, from the fits in Fig. 15 are summarized in Table 3. As Fig. 15 shows (see also Fig. S2 in the ESI $\dagger$ for the original data), the induction period shortens, and the autocatalytic growth portion of the curve steepens, as the temperature of the reaction mixture increases-just as one expects for a twostep process that separates, at least somewhat, nucleation from growth and for temperature-dependent nucleation, $k_{1}$, and autocatalytic surface growth, $k_{2}$, rate constants.

A crude activation parameter plot for just the 3 data points in Table 3 over that limited temperature range yields the approximate activation parameters $\Delta H^{\ddagger}=8.9 \pm 2.1 \mathrm{kcal} \mathrm{mol}^{-1}$ and $\Delta S^{\ddagger}=3.4 \pm 6.5$ e.u. (see Fig. S3 in the ESI $\dagger$ for the activation parameter plot; note also the somewhat unusual standard state for the $\Delta S^{\ddagger}$ of ("particles per volume") ${ }^{-1} \min ^{-1}$ ). ${ }^{5}$ The above kinetics data and analysis demonstrates that Turkevich's kinetics data for the formation of his colloidal $\mathrm{Au}(0)_{n}$ particles are inconsistent with the "burst nucleation" and "diffusioncontrolled growth" model. We have already cited Turkevich's 1953 "Organizer" mechanism ${ }^{6}$ and noted that Turkevich deserves credit for an early version of what now is the popular concept of prenucleation clusters or gels. ${ }^{72-77,81,175-178}$ 


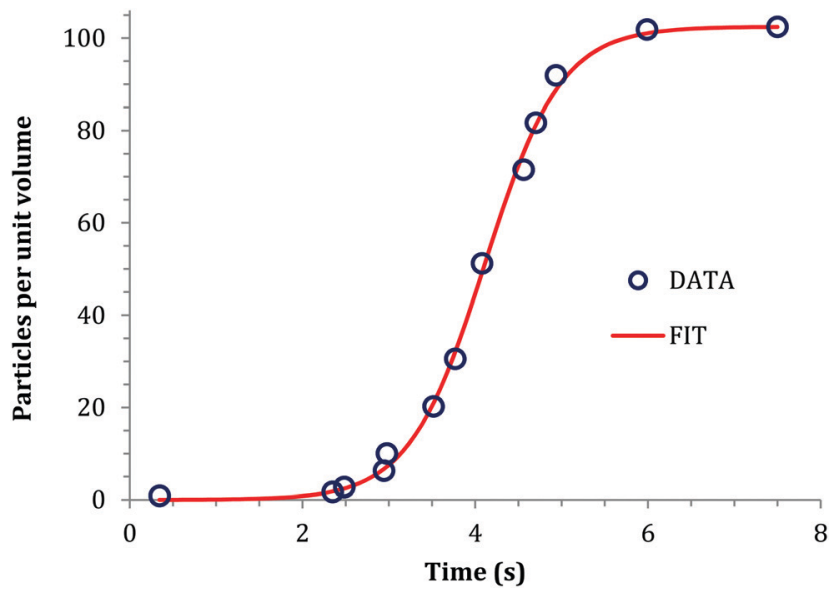

Fig. 16 Plot reproduced from Turkevich's 1951 paper $^{5}$ of the number of gold(0) nanoparticles per unit volume versus time during gold sol formation from a mixture of sodium citrate and tetrachloroauric acid by addition of hydroxylamine. Circles represents the experimental points measured using a nephelometer. The solid curve shows the fit of data to the 2-step mechanism ${ }^{3}$ of slow, continuous nucleation, $A \rightarrow B$, followed by autocatalytic surface growth, $\mathrm{A}+\mathrm{B} \rightarrow 2 \mathrm{~B}$ yielding the rate constants $k_{1}=(1.9 \pm 0.6) \times 10^{-4} \mathrm{~s}^{-1}$ and $k_{2}=(2.28 \pm 0.09) \times 10^{-2}$ (particles per unit volume $)^{-1} \mathrm{~S}^{-1}, R^{2}=0.99774$ and reduced Chi-Sqr $=3.58 \times 10^{-4}$. Fig. S4 in $\mathrm{ESI} \dagger$ presents the original data. ${ }^{5}$

Not surprisingly given the fits already presented in Fig. 15, it is possible to fit additional data from Turkevich's classic work ${ }^{5}$ with the 2-step mechanism, ${ }^{3}$ Fig. 16 and 17 . The resultant fits support the general concepts of slow continuous nucleation, and autocatalytic surface growth ${ }^{3}$ for the $\mathrm{Au}(0)_{n} /$ citrate system (Fig. 18).

In Fig. 17, Turkevich once again monitored the number of particles versus time, but now while using sodium acetonedicarboxylate as the reductant, the oxidized species of sodium citrate. ${ }^{6}$ The number of particles per unit volume is not instantaneous, and also increases with time, additional disproof of burst nucleation in his classic $\mathrm{Au}(0)_{n}$ nanoparticle formation system.

In short, Turkevich's classic 1951 and subsequent papers, plus the fits of the kinetics to a 2-step mechanism provided herein for the first time, disprove compellingly the burstnucleation and diffusion-controlled model as applied to Turkevich's classic $\mathrm{Au}(0)_{n}$ formation system. It is surprising that Turkevich's 1951 experimental evidence did little to dampen the enthusiasm for the burst nucleation theory in the intervening $\sim 70$ years.

Meriting mention at this point is a scholarly paper by Balashev and co-workers ${ }^{151}$ that compares the kinetics of $\mathrm{Au}(0)_{n}$ formation by five methods: UV-visible (plasmon resonance, including a proper analysis of the plasmon resonance), SEM, DLS, TEM, and AFM, the latter thereby introducing AFM to the toolbox of ways to monitor nanoparticle formation kinetics. ${ }^{151}$ Important conclusions from this notable work (which used the 2-step mechanism ${ }^{3}$ to analyze the observed kinetics) include (see Table 1 in their paper ${ }^{151}$ ) (i) that both UV-visible and AFM gave the same values of nucleation, $k_{1}$, and autocatalytic growth, $k_{2}$, rate constants within experimental

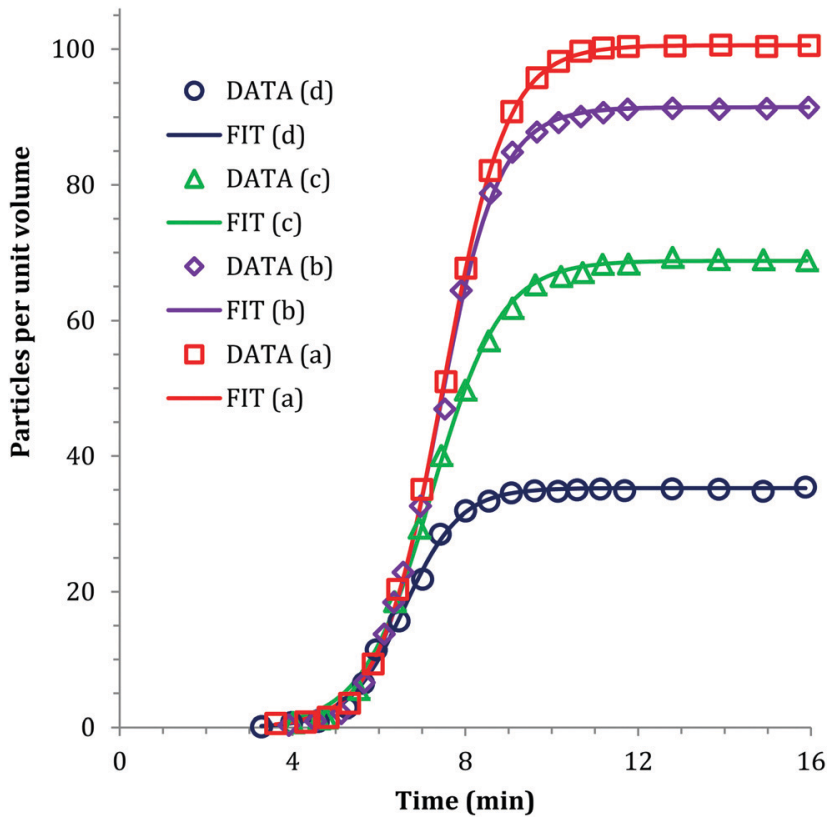

Fig. 17 Plot reproduced from Turkevich's 1951 paper $^{5}$ of the number of gold(0) nanoparticles per unit volume versus time during the gold sol formation from a mixture of sodium citrate and tetrachloroauric acid (a, red squares) nanoparticles obtained after complete development without adding hydroxylamine, and $(b-d$, purple diamonds, green triangles, dark blue circles) nanoparticles developed until addition of hydroxylamine at 6,7 , and 8 minutes. The solid curves show fits to a 2-step mechanism ${ }^{3}$ yielding the rate constants given in Table 4. See Fig. S5 in ESI $\dagger$ for the original data. ${ }^{5}$

error of both methods, but (ii) that TEM and DLS gave $k_{1}$ values differing by $\sim 0.16$ fold and $k_{2}$ differing by $\sim 2.1$ fold, while (iii) SEM failed for $\mathrm{Au}(0)_{n}$ nanoparticles $<20 \mathrm{~nm}$. Useful in terms of the context of the present review and the curve-fits shown in Fig. 15-17 is their conclusions that “...our findings support strongly. . .that Turkevich's 'organizer' word-only mechanism is really the FW 2-step mechanism and therefore should be replaced by the FW 2-step mechanism". ${ }^{151}$ Our added comment here is that while the 2-step mechanism can and does fit Turkevich's kinetics data, that in no way takes away anything from Turkevich's early studies and scholarly, formative contributions to gold nanoparticle chemistry and nanoparticle science more generally. ${ }^{5}$ And, the prenucleation cluster $^{72-77,81,175-178}$ notion contained in Turkevich's 1953 Organizer mechanism ${ }^{6}$ precedes by 67 years what is currently a hot topic ${ }^{72-77,81,175-178}$ in nanoparticle formation science. Additionally, there is other, more detailed work on the mechanism(s) of $\mathrm{Au}(0)_{n}$ nanoparticle formation, for example those in the next section due to Polte's group. The main point of the fittings above to the 2-step minimum mechanism is to test if “instantaneous nucleation" has any support in such $\operatorname{Au}(0)_{n}$ nanoparticle formation systems. It does not.

\subsection{Direct observation of gold particle formation via synchrotron $\mathrm{X}$-ray radiation techniques}

In the last decade, the use of synchrotron X-ray sources has become a preferred way to directly observe the process of 


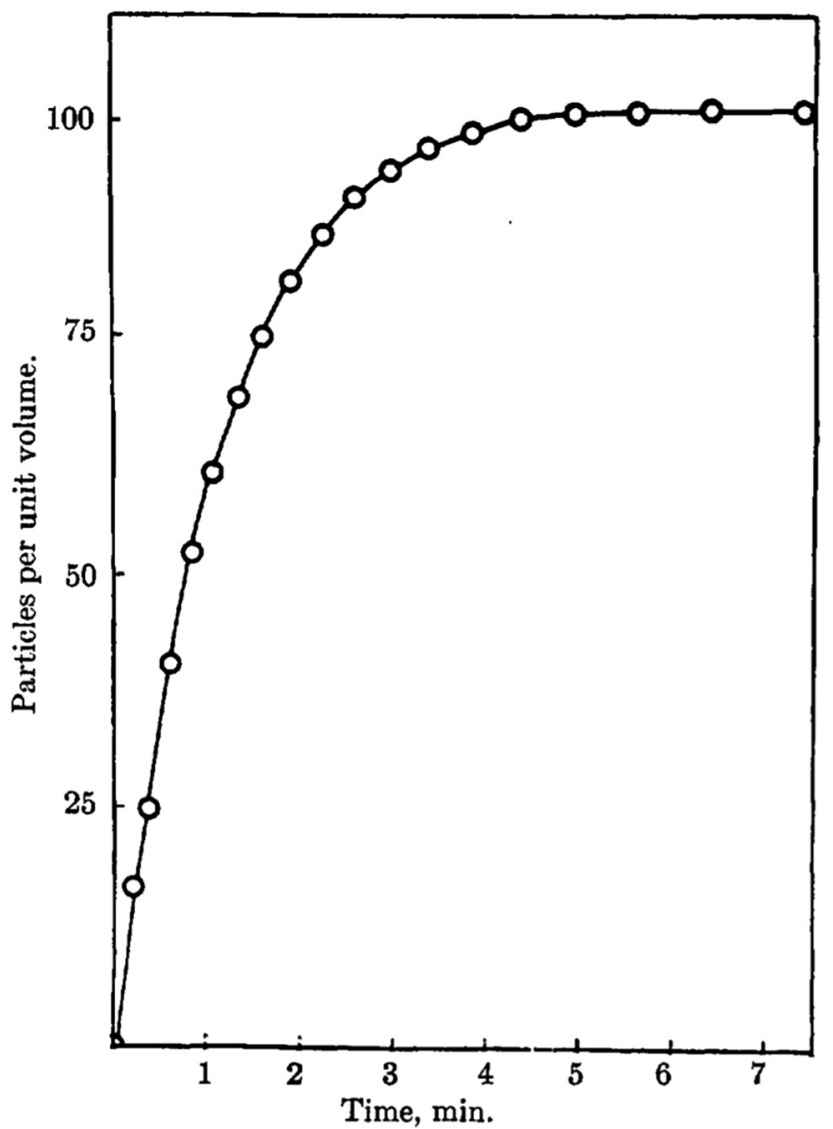

Fig. 18 (Fig. 3 from ref. 6.) The particles per unit volume versus time in minutes for the formation of colloidal gold from tetrachloroauric acid, $\mathrm{HAuCl}_{4}$ and sodium acetonedicarboxylate, $\mathrm{Na}_{2}\left[\mathrm{OC}\left(\mathrm{CH}_{2} \mathrm{COO}\right)_{2}\right]$. This curve provides direct evidence that new particles continue to appear over the first several minutes of the reaction. Reproduced with permission from ref. 6. Copyright 1953 American Chemical Society.

metal nanoparticle formation. The use of SAXS, ${ }^{179,180}$ WAXS, ${ }^{181}$ and XAFS ${ }^{182,183}$ provides exceptional detail on particle size, metal oxidation state, number of nearest neighbors, and the identity of those nearest neighbors, all in real-time. In our own, collaborative work on SAXS of Ir nanoparticle formation, an important control experiment reveals that even a small $\operatorname{Ir}_{4}$ (isolable, fully characterized, and hence authentic) cluster ${ }^{184,185} \operatorname{Ir}_{4} \mathrm{H}_{4}(1,5-\mathrm{COD})_{4}$ is readily detectable by synchrotron-based SAXS (A. Karim, C. B. Whitehead, R. G. Finke, collaborative unpublished results). That control alone strongly supports the thesis that synchrotron-based SAXS ${ }^{179,180}$ holds considerable promise for adding to our insights about especially nucleation, but also growth and agglomeration, of nanoparticles.

Polte and co-workers have reported several noteworthy, in-depth studies of Turkevich's classical citrate reduction of tetrachloroauric acid, measured by tandem in situ XANES and SAXS (along with some UV-vis), resulting in time-resolved, direct observation of gold particle formation. ${ }^{169-171}$ As seen in Fig. 19, the number of particles and hence nucleation is, once again, not instantaneous. ${ }^{169}$ Additionally, the number of particles decreases implying particle coalescence/agglomeration occurs later in the reaction and under the conditions employed. Polte et al. ${ }^{170}$ have also reported that the number of particles increases at the beginning of the reaction (observable due to the excellent time-resolution within the first seconds of the reaction, Fig. 5b therein), thereby again disproving "burst" nucleation in at least the specific gold particle formation system studied.

Polte's valuable studies ${ }^{169,170}$ provide not only direct, synchrotron-radiation-based, additional disproof of the burst nucleation and diffusion-controlled growth model for $\mathrm{Au}(0)_{n}$ nanoparticle formation, they also reveal the need for increased time resolution during the first 5 minutes of the reaction so as to better capture nucleation. ${ }^{186}$ These workers claim, however, that the necessary models to describe quantitatively the formation and growth of particles do not exist. Actually, disproof-based, deliberately minimalistic mechanistic models do in fact exist ${ }^{3,17,26,27,55-58,60-62}$ as described back in Table 2, mechanisms that can be applied quantitatively (i.e., as used in Fig. 15-17), including mechanisms that can quantitatively describe aggregation and coalescence. ${ }^{55-58}$ Indeed, it has already been noted that there are now 5 classes and 96 distinct pseudo-elementary step mechanisms that one can use to try to fit quantitatively one's particle formation kinetics data. The $\operatorname{Ir}(0)_{n}$ system that gave rise to a number of those mechanisms is examined next, but only briefly, because it is our own work and because multiple full papers describing the systems and the kinetics and mechanistic evidence are available to the interested reader. ${ }^{3,17,26,27,55-58,60-62}$

\subsection{Iridium nanoparticles and the origin of the 2-, 3- and two 4-step mechanisms}

Iridium $(0)_{n}$ nanoparticles prepared from an atomically precisely defined precursor $\left\{(1,5-\mathrm{COD}) \mathrm{Ir}^{\mathrm{I}} \cdot \mathrm{POM}\right\}^{8-}$, and hence stabilized by a novel $\left[\mathrm{P}_{2} \mathrm{~W}_{15} \mathrm{Nb}_{3} \mathrm{O}_{62}\right]^{9-}$ polyoxometalate (POM) "Gold Standard" stabilizer, ${ }^{187}$ led to the 2 -step mechanism for particle formation, nominally: $\mathrm{A} \rightarrow \mathrm{B}$, then $\mathrm{A}+\mathrm{B} \rightarrow 2 \mathrm{~B}$, where $\mathrm{A}$ really is $\mathrm{A}=\mathrm{A} \cdot \mathrm{L}(\mathrm{L}=$ ligand $)=$ the $\left\{(1,5-\mathrm{COD}) \mathrm{Ir}^{\mathrm{I}} \cdot \mathrm{POM}\right\}^{8-}$

Table 4 The rate constants $k_{1}$ for slow, continuous nucleation and $k_{2}$ for autocatalytic surface growth for the formation of gold sols from a sodium citrate and tetrachloroauric acid mixture developed by addition of hydroxylamine hydrochloride

\begin{tabular}{lllll}
\hline Data & Addition time of hydroxylamine & $k_{1}, \min ^{-1}$ & $k_{2}$, (particles per volume) ${ }^{-1} \mathrm{~min}^{-1}$ & $R^{2}$ \\
\hline (a) & No addition & $(4.6 \pm 0.6) \times 10^{-5}$ & $1.38 \pm 0.02$ & 0.99972 \\
(b) & 6 min & $(3.7 \pm 0.9) \times 10^{-5}$ & $1.58 \pm 0.04$ & $5.02 \times 10^{-5}$ \\
(c) & 7 min & $(1.5 \pm 0.3) \times 10^{-4}$ & $1.83 \pm 0.05$ & 0.99899 \\
(d) & 8 min & $(6.0 \pm 2.1) \times 10^{-5}$ & $4.21 \pm 0.17$ & 0.99887 \\
\end{tabular}




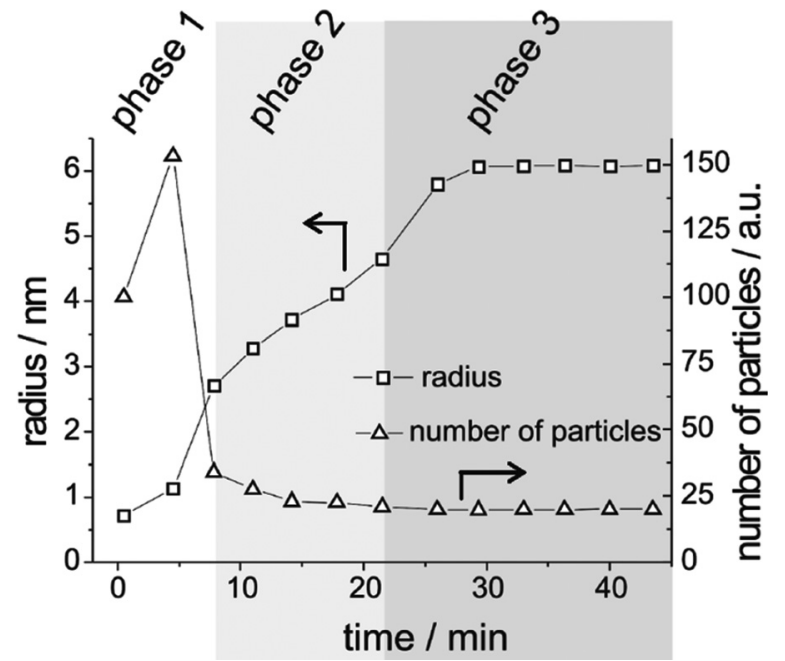

Fig. 19 (Fig. 3d from ref. 169.) The number of particles versus time (in minutes; represented by triangles) showing an initial increase followed by a dramatic decrease, at $75{ }^{\circ} \mathrm{C}$ and for $0.375 \mathrm{mM}$ auric acid plus $2.5 \mathrm{mM}$ sodium citrate and as measured by SAXS and XANES. The squares represent the radius (in nanometers) versus time (in minutes) measured in tandem with the number of particles. Reproduced with permission from ref. 169. Copyright 2010 American Chemical Society.

precursor or precatalyst, and $\mathrm{B}=$ the growing $\operatorname{Ir}(0)_{n}\left(\mathrm{POM}^{9-}\right)_{m}$ prototype system. ${ }^{3}$ The balanced-equation defined $k_{1}$ and $k_{2}$ rate constants, and even more importantly the balancedequation, mechanistically defined words to describe the process accurately, slow, continuous nucleation, followed by autocatalytic surface growth, are important aspects of the 2-step mechanism-the first alternative in nearly 50 years $^{3}$ to the 1950 burst-nucleation model. ${ }^{1}$ Available to the interested reader is a discussion of what turned out to be the five special features ${ }^{27}$ of the prototype $\left\{(1,5-\mathrm{COD}) \mathrm{Ir}^{\mathrm{I}} \cdot \mathrm{POM}\right\}^{8-}$ precursor and $\operatorname{Ir}(0)_{n}\left(\mathrm{POM}^{9-}\right)_{m}$ nanoparticle product system and the strengths, but also the weaknesses, ${ }^{48,60,89}$ of the deliberately minimalistic, Ockham's razor obeying, disproof-based 2-step mechanism. A 3- and two 4-step mechanisms are also available that are extensions of the 2-step mechanism that include bimolecular agglomeration $(\mathrm{B}+\mathrm{B} \rightarrow \mathrm{C}),{ }^{55,188}$ autocatalytic agglomeration $(\mathrm{B}+\mathrm{C} \rightarrow 1.5 \mathrm{C}){ }^{56-58,188}$ and secondary autocatalytic surfacegrowth $(\mathrm{A}+\mathrm{C} \rightarrow 1.5 \mathrm{C}) .^{60-62}$

Already mentioned in connection with mechanism-enabled population balance modeling (ME-PBM) ${ }^{61,62}$ is the discovery of a new, 3-step minimalistic mechanism that is a simple, but key, one-step extension of the classic 2-step minimum mechanism in which the $\mathrm{A}+\mathrm{B} \rightarrow 2 \mathrm{~B}$ growth step is broken up into two sizedependent growth step. That new 3-step mechanism that was discovered via ME-PBM is the pseudo-elementary step mechanism of $\mathrm{A} \rightarrow \mathrm{B}$ (rate constant $k_{1}$ ), $\mathrm{A}+\mathrm{B} \rightarrow \mathrm{C}$ (rate constant $k_{2}$ ), and $\mathrm{A}+\mathrm{C} \rightarrow 1.5 \mathrm{C}$ (rate constant $k_{3}$ ). The fact that the new 3-step mechanism can account for relatively narrow PSDs, via the finding that $k_{2}>k_{3}$ so that the smaller particles, $\mathrm{B}$, grow faster than larger particles, $\mathrm{C},{ }^{61,62}$ explains how one can have slow, continuous nucleation ${ }^{3}$ yet still obtain narrow PSDs. Note that despite the general, somewhat ill-defined nature (i.e., outside of the atomistic ME-PBM) of the "smaller" and "larger" particles B and C respectively, the 3-step pseudoelementary step model was sufficient to (a) discover the critical $k_{2}>k_{3}$, "smaller react faster than larger" finding and concept for getting to near-monodisperse PSDs despite continuous nucleation; and (b) to permit MATLAB code to be written that allows a quantitative fitting of the PSDs with extraction of the rate constants from PSD data. The new 3-step in turn obviates the need to postulate "instantaneous/burst" nucleation ${ }^{1}$ in order to obtain narrow PSD from self-assembly reactions starting from continuous nucleation ${ }^{3}$ followed by thousands of chemical steps. ${ }^{61,62}$

\subsection{Use of synchrotron $X-$ ray radiation techniques to monitor the formation of platinum, silver, and palladium nanoparticles in separate studies}

Several researchers have conducted kinetics studies of Pt, Pd, or Ag nanoparticle formation using either small-angle X-ray scattering (SAXS) or X-ray absorption fine structure (XAFS), while also mentioning the 1950 model in their papers. A list of such studies is given as Table S3 of the ESI. $\dagger$ Next, we describe three such illustrative studies on platinum, silver, and palladium nanoparticles.

5.4.1. Platinum nanoparticles. In 2012, Harada and Kamigaito used Quick-XAFS (QXAFS) to study the nucleation and aggregative growth process of platinum nanoparticles ${ }^{189}$ formed by the photoreduction of hexachloroplatinic(Iv) acid in ethanol with polyvinylpyrolidone (PVP, $\left.M_{\mathrm{W}}=40000\right)$ as a particle stabilizer. In situ monitoring at the $\mathrm{Pt} \mathrm{L}_{3}$-edge and acquisitions were performed at 10-20 second intervals. Fig. 20 displays (a) the absorbance versus time, (b) the normalized absorbance versus time, (c) plots of $\left[\mathrm{Pt}^{4+}\right]_{t} /\left[\mathrm{Pt}^{4+}\right]_{0}$ and $\left[\mathrm{Pt}^{2+}\right]_{t} /$ $\left[\mathrm{Pt}^{2+}\right]_{500}$ versus time, and $(\mathrm{d})\left[\mathrm{Pt}^{2+}\right]_{t} /\left[\mathrm{Pt}^{2+}\right]_{500}$ and $\left[\left(\mathrm{Pt}^{0}\right)_{m}\right]_{t} /$ $\left[\left(\mathrm{Pt}^{0}\right)_{m}\right]_{6000}$ versus time.

As seen in Fig. 20, Harada and Kamigaito directly monitored both the loss of platinum precursor and the formation of platinum nanoparticles by QXAFS. ${ }^{189}$ They observe an induction period at the beginning of irradiation followed by autocatalytic growth period. During the latter half of the growth period, the growth appears to be linear, which Harada assigns to be Ostwald ripening. The data were fit using differential equations from two nucleation and growth models: the AvramiErofe'ev (A-E) semi-empirical model ${ }^{42-44}$ and the 2-step mechanism. ${ }^{3}$ Both the A-E model and the 2-step mechanism fit the kinetics data (i.e., up to the point where linear growth is seen that is attributed by the authors to Ostwald Ripening). The equivalent fits by the A-E and 2-step models are not surprising considering the 2-step mechanism has been shown to be the minimal chemical mechanism underlying the semi-empirical Avrami equation. ${ }^{48}$ Use of the 2 -step mechanism does, however, allow physically meaningful rate constants for nucleation and autocatalytic growth to be obtained rather than the Avrami exponent, $\mathrm{n}$, and $\mathrm{k}$ parameters, ${ }^{48}$ the interpretation of which remains controversial. ${ }^{48,51}$ The fits by the A-E and 2-step models in turn implies that the burst nucleation model cannot account for the QXAFS data acquired by Harada and Kamigaito. 

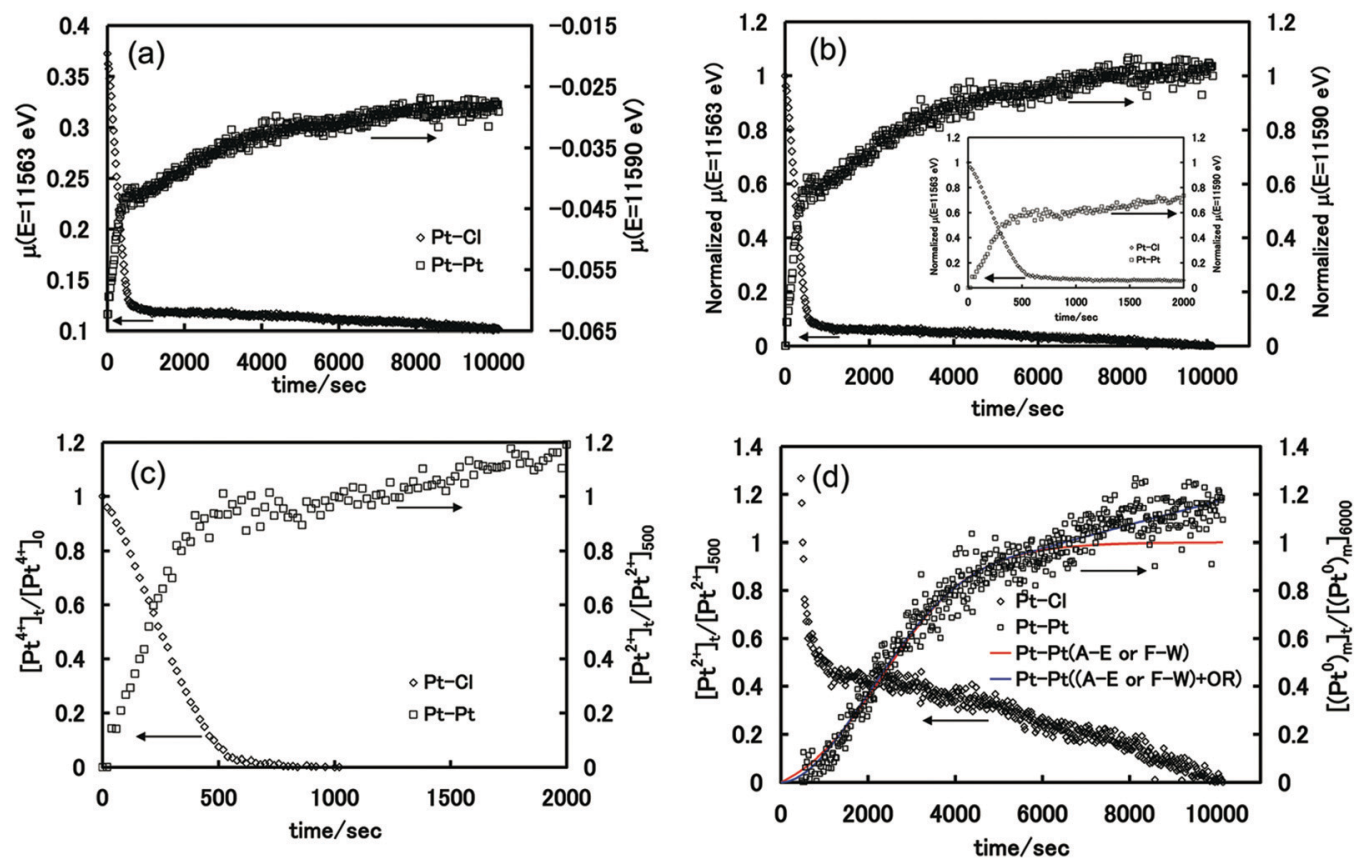

Fig. 20 (Fig. 4a-d from ref. 189.) (a) Time evolution of the absorbance coefficient, $\mu(E)$, intensity at the intrinsic peak energy (11563 and 11590 eV) for the colloidal dispersions of Pt nanoparticles ( $[\mathrm{Pt}]=24.4 \mathrm{mM})$. (b) Time evolution of the normalized $\mu(E)$ as a function of the reduction time $(t)$. The inset displays the corresponding enlarged plots during the first $2000 \mathrm{~s}$. (c) Plots of $\left[\mathrm{Pt}^{4+}\right]_{t} /\left[\mathrm{Pt}^{4+}\right]_{0}$ and $\left[\mathrm{Pt}^{2+}\right]_{t} /\left[\mathrm{Pt}^{2+}\right]_{500}$ as a function of the reduction time on the basis of the temporal change of the normalized $\mu(E=11563 \mathrm{eV})$ and $\mu(E=11590 \mathrm{eV})$. (d) Plots of $\left[\mathrm{Pt}^{2+}\right]_{t} /\left[\mathrm{Pt}^{2+}\right]_{500}$ and $\left[\left(\mathrm{Pt}^{0}\right)_{m}\right]_{t} /\left[\left(\mathrm{Pt}^{0}\right)_{m}\right]_{6000}$ as a function of the reduction time. Curve fitting to the experimental data was carried out on the basis of the Avrami-Erofe'ev (A-E) or Finke-Watzky (FW) 2-step model (red curve) for the solid-state kinetics or for the solution-phase reaction kinetics, and the A-E or FW model plus Ostwald ripening (OR) (blue curve). Reproduced with permission from ref. 189. Copyright 2012 American Chemical Society.

5.4.2. Silver nanoparticles. In 2014 , Yan et al. published an important paper highlighting the growth behavior of silver nanoparticles monitored by SAXS. ${ }^{190}$ Silver nanoparticles were prepared from silver nitrate solutions reduced by the strongly reducing reductant sodium borohydride and stabilized by polyvinylpyrrolidone (PVP). Yan and co-workers used in situ, time-resolved SAXS to obtain excellent time-resolved data throughout the reaction, including at the beginning of the reaction during the induction period, as shown in Fig. 21.

During the first seconds of the reaction, Yan and co-workers observed a rapid increase in the number of silver particles due to the use of the strong reductant, $\mathrm{NaBH}_{4}$. Yan and co-workers note that "the nucleation begins at the very early stage, but it probably lasts in the whole formation process" $" 190$ while also commenting on the inability of the 1950 model to explain their kinetics data. The observed decrease in the number of particles is prima facie evidence for some type of coalescence/ agglomerative process.

However, this study uses a diffusion-coalescence growth model, proposed on the basis of the assumption that Brownian motion drives the $\mathrm{Ag}$ particles to diffuse in the suspension and collide with each other to coalesce together $\left(\mathrm{Ag}+\mathrm{Ag} \rightarrow \mathrm{Ag}_{2}\right.$, $\left.\mathrm{Ag}_{m}+\mathrm{Ag}_{n} \rightarrow \mathrm{Ag}_{m+n}, \ldots\right)$ and associated eqn (13) to fit the particle size $v s$. time data, Fig. 21, vide supra,

$$
r=r_{\mathrm{c}}+\frac{r_{0}}{b}\left(C_{\mathrm{Ag}} N_{\mathrm{A}}\right)^{\frac{1}{3}} \ln \left[1+\frac{8 b r_{0}}{3 r_{\mathrm{c}}} Q\left(r_{\mathrm{c}}\right)\left(C_{\mathrm{Ag}} N_{\mathrm{A}}\right)^{\frac{1}{3}}\left(t-t_{\mathrm{c}}\right)\right]
$$

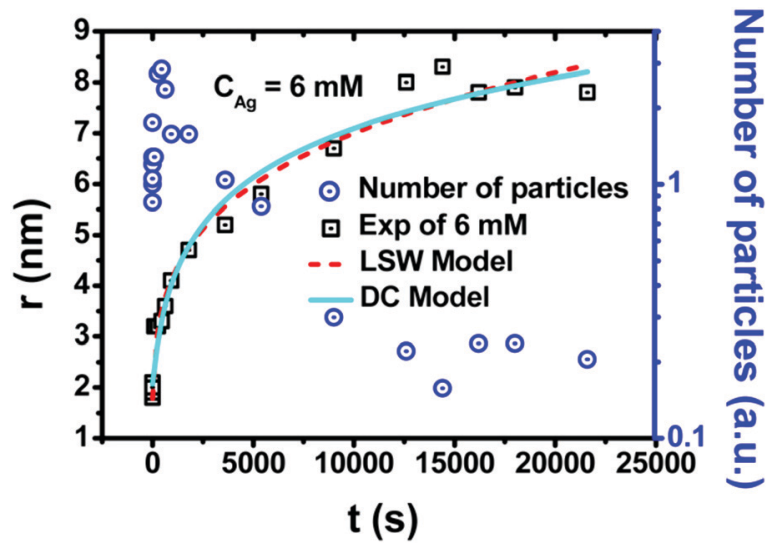

Fig. 21 (Fig. 6 from ref. 190.) Particle number (a.u.) and particle size (nanometer) are plotted versus reaction time (seconds) at $6 \mathrm{mM} \mathrm{AgNO}_{3}$. The size vs. time data and the particle number vs. time data are represented, respectively, by black squares and blue circles. The size versus time data were fit with two different growth models. The dashed red line is the LSW (Lifshitz, Slyozov, and Wagner) Model proposing a mean-field theory of Ostwald ripening, and the solid, light blue line is the DC (DiffusionCoalescence) Model of growth, discussed below. Reproduced with permission from ref. 190. Copyright 2014 American Chemical Society.

where $r$ is the particle radius, $r_{\mathrm{c}}$ is a critical particle radius, $r_{0}$ is the particle radius at time zero (the radius of the seeds at the start of the growth period), $b$ a probability decay factor, $C_{\mathrm{Ag}}$ is the initial concentration of silver in suspension, $N_{\mathrm{A}}$ is 
Avogadro's number, $t$ is time, $t_{\mathrm{c}}$ is time it takes to form the putative critical particle, and $Q\left(r_{\mathrm{c}}\right)$ is a function of both $D$ (the diffusion coefficient) and $z$ (the "coalescence" factor). This equation can fit the size versus time data in Fig. 21, and the modeling yields values for three parameters, the "coupling factor" $D(z-1)$, the "probability decay factor" $(b)$, and the "critical particle radius" $\left(r_{\mathrm{c}}\right)$. Unfortunately, these three parameters yield little in terms of chemically and physically meaningful insights for a process that is, chemically, a nucleation, growth, and agglomeration process - the presence of agglomeration being implied by the drop in the number of particles seen in Fig. 21. Moreover, postulation of a "critical particle radius" is a concept analogous to the "critical nucleus" from CNT, which by all indications is inapplicable to strong bonding systems ${ }^{17}$ where the nucleating species loses its molecular identity (i.e., the identity of a $\operatorname{Ag}(0)$ atom) in an $\operatorname{Ag}(0)_{n}$ nucleus arguably better conceptualized as the kinetically effective nucleus. ${ }^{17}$ Moreover, recent evidence exists for very low, $n=2-3$ molecularity, ${ }^{17,26,27}$ readily measurable ${ }^{17,26,27}$ kinetically effective nuclei, ${ }^{17} \mathrm{M}_{n}(\mathrm{M}=$ transition metal)-in contradistinction to the theoretical, highest energy species, hence not actually detectable ${ }^{28}$ "critical nucleus" concept of CNT. Yan and co-workers also conclude that the 1950 model cannot be used quantitatively to fit their data and is inappropriate for their system, but then err in our opinion in borrowing the critical nucleus concept from CNT. Overall, Yan et al. have produced superb data with compelling evidence against the 1950 model. But, the task of describing and fitting their data by a chemical reaction mechanism remains to be accomplished.

A very recent, important account by Bigioni and his coworkers $^{158}$ covers the synthesis of silver molecular nanoparticles in some detail noting the importance of coordinating ligands and thermodynamic stability of $\mathrm{Ag}_{44}$ nanoparticles. The $\mathbf{M}_{4} \mathrm{Ag}_{44}(p-\mathrm{MBA})_{30}$ nanoclusters are prepared from the sodium borohydride reduction of $\mathrm{Ag}(\mathrm{I})-p$-MBA precursor ( $p$-MBA: $p$-mercaptobenzoic acid) in the presence of alkali metal cation $\left(\mathrm{M}^{+}\right)$at $\mathrm{pH}=9$ in ethanol-water solution. ${ }^{191}$ The authors hypothesize the time of nucleation does not affect the size of the nanoparticle product, based on the results obtained from a test of separating nucleation events by arbitrarily long periods of time, and then evaluating the effect on the product size distribution. ${ }^{158}$ This test was performed by preparing a reaction mixture, splitting it into two parts, reducing only one of the two parts to obtain the $\mathrm{Ag}_{44}$ product, and then mixing the two parts back together such that the second part of the reaction mixture could be reduced in the presence of the $\mathrm{Ag}_{44}$ product (which had nucleated an arbitrary time earlier). Normally, one would have expected the $\mathrm{Ag}_{44}$ nanoparticles added to the reaction mixture to have acted as seeds and grown as the reducing agent was added, at least in the absence of very strong-binding ligands. The authors clearly demonstrated this was not the case; instead, the product is identical to that of the standard preparation as seen in Fig. 3 of their paper. ${ }^{191}$ The interesting, discrete $\mathrm{Ag}_{44}$ system merits additional mechanistic study and ME-PBModeling. ${ }^{61,62}$
5.4.3. Palladium nanoparticles. In a recent, important paper from A. Karim's laboratories, ${ }^{63}$ in situ SAXS measurements and kinetic modeling using a modified and expanded form of the 2-step mechanism (Table 2, vide supra) were combined to quantitatively capture the role of ligand-metal binding (i.e., ligand to nanoparticle surface metal site) as an important factor in controlling the synthesis kinetics of the $\left\{\mathrm{Pd}(0)_{n}\left(\mathrm{POct}_{3}\right)_{a}(\mathrm{OAc})_{b}\right\}^{b-}$ nanoparticles (formed from the reduction of palladium(II) acetate in the presence of trioctylphosphine in two different solvents toluene and pyridine, as mixtures with hexanol). The results reveal the role of ligands and solvents in controlling the rates of both nucleation and growth and, consequently, the final nanoparticles size. The SAXS measurements provide the evolution of number and size of the $\left\{\mathrm{Pd}(0)_{n}\left(\mathrm{POct}_{3}\right)_{a}(\mathrm{OAc})_{b}\right\}^{b-}$ nanoparticles as redisplayed in Fig. 22, compelling evidence against the 1950 model assumptions of a burst nucleation and putative constant number of particles. ${ }^{1}$ Similar to many previous observations, ${ }^{192-195}$ the overlap of nucleation and growth are also apparent in Fig. 22. For example, in toluene, as the reaction time increased from 1250 to 9600 seconds, the number of particles per liter of solution doubles (from $3 \times 10^{19}$ to $6 \times 10^{19} \mathrm{~L}^{-1}$ ) while the particle size increased only from $\sim 1.0$ to $1.3 \mathrm{~nm}$.

While the evidence refuting the 1950 model for the formation of $\left\{\operatorname{Pd}(0)_{n}\left(\mathrm{POct}_{3}\right)_{a}(\mathrm{OAc})_{b}\right\}^{b-}$ nanoparticles is an important and definitive part of the noteworthy study by A. Karim and co-workers, ${ }^{63}$ the most important part of that work is that (i) it supports and extends significantly the addition to the 2-step mechanism of a A.L $\rightleftharpoons \mathrm{A}+\mathrm{L}$ prior equilibrium first documented in 1997 in ref. 3 and then in more detail in 2017 in ref. 26 and then in 2019 in ref. 27, and very importantly (ii) it is the

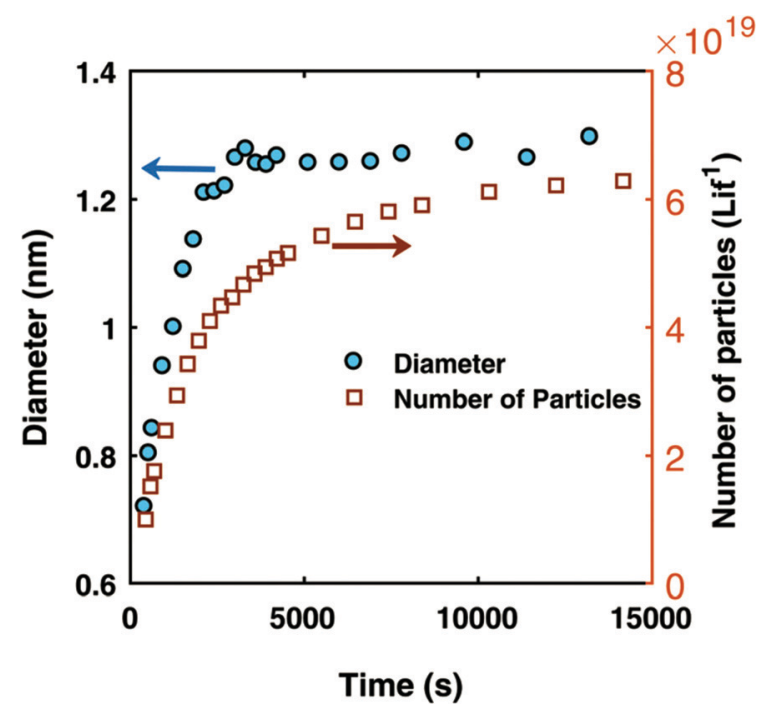

Fig. 22 (Fig. 1b from ref. 63.) The number of particles per liter of solution and the average particle size (nanometer) are plotted versus reaction time (seconds) obtained via in situ SAXS measurement for the formation of $\left\{\mathrm{Pd}(0)_{n}\left(\mathrm{POCt}_{3}\right)_{a}(\mathrm{OAc})_{b}\right\}^{\text {b- }}$ nanoparticles starting with $10 \mathrm{mM} \mathrm{Pd}(\mathrm{OAc})_{2}$ plus 2 equivalents of trioctylphosphine per mole of $\mathrm{Pd}$ in 50:50 toluene: hexanol mixture at $100{ }^{\circ} \mathrm{C}$. Reproduced with permission from ref. 63 . Copyright 2017 The Royal Society of Chemistry. 
first compelling evidence for the addition of the B.L $\rightleftharpoons \mathrm{B}+\mathrm{L}$ equilibrium to the 2-step mechanism. In short, the classic 2-step mechanism is thereby expanded to a 4-step, prior- and post-equilibria mechanism of nominally: $\mathrm{A} \cdot \mathrm{L} \rightleftharpoons \mathrm{A}+\mathrm{L}, \mathrm{A} \rightarrow \mathrm{B}$, $\mathrm{B} \cdot \mathrm{L} \rightleftharpoons \mathrm{B}+\mathrm{L}$, and then $\mathrm{A}+\mathrm{B} \rightarrow 2 \mathrm{~B}$.

Harada and co-workers ${ }^{196}$ reported the formation of palladium (and rhodium) nanoparticles from photoreduction of metal chloride precursors in aqueous ethanol solution in the presence of polyvinylpyrrolidone (PVP) polymer stabilizer, which was followed by UV-vis, TEM, and in situ XAFS. Kinetics data are shown to fit to the 2-step mechanism for the formation of metal nanoparticles. ${ }^{196}$ A recent study on the palladium nanoparticle formation from the photoreduction of $\left[\mathrm{PdCl}_{4}\right]^{2-}$ precursor using the same methodology has also been reported and reaches similar conclusions. ${ }^{197}$

In short, considerable, direct, compelling evidence against-but no evidence for-the burst-nucleation model exists from studies of the formation of $\mathrm{Au}, \mathrm{Ir}, \mathrm{Pt}, \mathrm{Ag}$, and $\mathrm{Pd}$ nanoparticles. An important, likely much more general finding that has come out of the metal(0) nanoparticle formation studies described is the extension of the 2-step mechanism ${ }^{3}$ to the 4-pseudo-elementary-step mechanism that includes ligand effects ${ }^{63,198}$ of $\mathrm{A} \cdot \mathrm{L} \rightleftharpoons \mathrm{A}+\mathrm{L}, \mathrm{A} \rightarrow \mathrm{B}, \mathrm{B} \cdot \mathrm{L} \rightleftharpoons \mathrm{B}+\mathrm{L}$, and then $\mathrm{A}+\mathrm{B} \rightarrow 2 \mathrm{~B}$.

\section{A closer look at other systems employing the 1950 model: the formation of oxide nanoparticles}

The 1950 model has also been used to try and understand the controlled growth of near-monodisperse silica spheres of micrometer size. Table S4 of the ESI $\dagger$ provides a summary list of 39 silica, titania, iron oxide, and other element-oxide nanoparticle formation papers that have considered the applicability or inapplicability of the 1950 model to the formation of oxide nanoparticles as those papers strove to explain their observed, sometimes record-narrow particle size-distributions. As before, prereading and prestudy of Table $\mathrm{S} 4$ (ESI $\dagger$ ) is recommended prior to proceeding with the next section.

\subsection{Stöber process for the formation of silica nanospheres}

A classic system describing the formation of "monodisperse" (i.e., actually not monodisperse, but very narrow size dispersion) particles was reported by Stöber, Fink and Bohn in 1968. ${ }^{199}$ The method, known as the "Stöber Process", involves the hydrolysis of a dilute solution of tetraethoxysilane (TEOS) at high $\mathrm{pH}$ in alcohol solvent to form spherical silica particles of almost identical size with diameters that vary from $0.01-0.5 \mu \mathrm{m}$ (10-500 nm) depending on $\mathrm{pH}$ and concentration of precursor used. Since the formative study by Stöber and his co-workers in $1968,{ }^{199}$ the synthesis of colloidal spherical silica nanoparticles has relied extensively on ammonia-catalyzed hydrolysis of TEOS, and subsequent condensation of the resulting silanols, with the resulting silica particles readily tuned in size from 10 to $500 \mathrm{~nm}$ simply by altering the ammonia concentration. ${ }^{200}$
The 1968 paper by Stöber, Fink and Bohn is one of the most cited publications in the field (at present $>10080$ citations according to SCI, June 2019). There is accordingly an enormous, important literature of the Stöber Process. Only those papers that specifically mention the 1950 model are covered in what follows. The reader interested in further insights into the intriguing and important Stöber Process should consult that original literature for all of its additional insights.

What is clear, however, is that despite a great deal of effort in the last decades, the formation mechanism of Stöber silica particles is still not well understood. Two growth models exist at present, the monomer-addition model $^{201}$ and an aggregation-only model, ${ }^{202}$ both of which assume burst nucleation-that is, neither model gives any type of adequate, experimentally based accounting of the critical, initial nucleation event(s). Additionally, both models fail to explain the huge, controllable variation in size and particle-size distribution of the resultant silica particles as a function of the ammonia concentration. Only the aggregative model has been claimed to be able to account for very narrow particle-size distributions, as discussed in the next section that addresses Zukoski's important Aggregative Growth Model.

The basic reactions occurring in the Stöber Process are generally agreed to be: ${ }^{203}$

$$
\begin{aligned}
\mathrm{Si}(\mathrm{OR})_{4}+x \mathrm{H}_{2} \mathrm{O} \rightarrow \mathrm{Si}(\mathrm{OR})_{4-x}(\mathrm{OH})_{x}+x \mathrm{ROH} \\
\mathrm{Si}(\mathrm{OR})_{4}+\mathrm{Si}(\mathrm{OR})_{4-x}(\mathrm{OH})_{x} \rightarrow(\mathrm{OR})_{7-x}(\mathrm{Si}-\mathrm{O}-\mathrm{Si})_{1}(\mathrm{OH})_{x-1} \\
+\mathrm{ROH}
\end{aligned}
$$

$$
2 \mathrm{Si}(\mathrm{OR})_{4-x}(\mathrm{OH})_{x} \rightarrow(\mathrm{OR})_{8-2 x}(\mathrm{Si}-\mathrm{O}-\mathrm{Si})_{1}(\mathrm{OH})_{2 x-2}+\mathrm{H}_{2} \mathrm{O}
$$

Note that equations analogous to (15) and (16) can also be written for the higher Si-containing silanols that are expected to be present.

Notable upfront is a 1993 paper $^{8}$ by Matijevic that reviewed the preparation of "monodisperse" particles of simple or mixed composition and different shapes such as spheres, cubes, rods and ellipsoids. Matijević surprised the community at the time by remarking that "the recognition of the limitations of the 1950 model came through a number of experimental findings that contradicted it" in a limited number of cases". 8 Additionally, Matijevic noted that "it has been proven in several cases for nucleation to be an ongoing process, yet still 'monodispersed' colloids were generated."8 Matijević then discusses a number of alternative pathways for the formation of colloidal particles that are summarized in Table S4 of the ESI. $\dagger$

6.1.1. Evidence supporting a rate-determining step of tetraethoxysilane hydrolysis. In a 1992 study of the formation of Stöber silica particles, the hydrolysis of tetraethoxysilane was monitored by ${ }^{13} \mathrm{C}$ NMR spectroscopy (Fig. 23) and the particle growth was monitored by time-resolved static light scattering (Fig. 5 of the 1992 paper). ${ }^{204}$ Both processes were found to have 

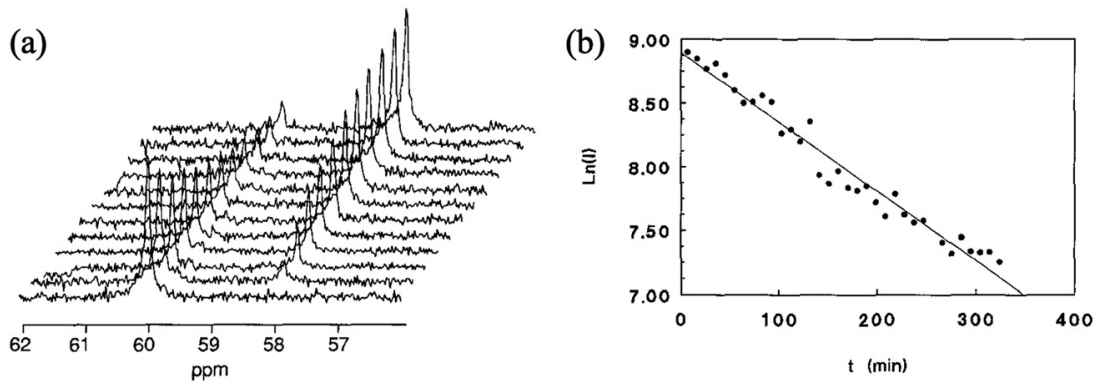

Fig. 23 (Fig. 3 and 4 from ref. 204.) (a) ${ }^{13} \mathrm{C}$ NMR peaks of the carbon bonded to oxygen and belonging either to an ethoxy group of TEOS molecule $(60.8 \mathrm{ppm})$, or to a hydrolyzed ethanol molecule $(58.8 \mathrm{ppm})$, as a function of time. The time difference between each scan is $28.8 \mathrm{~min}$, [TEOS] $=0.167 \mathrm{M}$, $\left[\mathrm{NH}_{3}\right]=0.668 \mathrm{M}$, and $\left[\mathrm{H}_{2} \mathrm{O}\right]=1.80 \mathrm{M}$ in propanol solution. (b) Logarithm of the integrated intensity of the ethoxy carbon ${ }^{13} \mathrm{C}$ NMR signal versus time. Reproduced with permission from ref. 204. Copyright 1992 Elsevier.

the same first-order rate constant; therefore, the overall rate of the particle formation looks to be limited by the first-order hydrolysis rate of the alkoxide - an unfortunate part of the Stöber process that hides the kinetics of the critical initial nucleation process, at least under the temperature and other conditions examined.

In the 2017 study $^{203}$ the concentration of TEOS was determined by ammonium molybdate spectrophotometric measurement throughout in the course of the Stöber process reaction starting with various ammonia concentrations. Conductivity of the reaction medium was also measured to reflect the concentration of anionic silanol intermediates, which exist in deprotonated, anionic and hence conducting form at $\mathrm{pH}>9$. An important finding is that unhydrolyzed TEOS molecules remain in the reaction medium until the end of process. Additionally, silanol species that are potential intermediates were identified by mass spectrometry, results which support similar findings of putative intermediates in silica particle formation detected by trimethylsilylation and then GC determination of the products. ${ }^{205}$ From the evaluation of TEOS concentration and conductivity versus time data, depending on the ammonia concentration, rate constants of TEOS hydrolysis were calculated and those of the subsequent condensation were derived by using the steady-state and equilibrium approximations. ${ }^{203}$ Based on the rate constants, the authors concluded that hydrolysis of one ethoxy group of TEOS molecule to form monovalent anionic silanol, $\mathrm{Si}(\mathrm{OEt})_{3}\left(\mathrm{O}^{-}\right)_{1}$, is the rate-limiting step for TEOS hydrolysis. Hence, TEOS serves as the precursor undergoing direct hydrolysis (eqn (14)), as well as being able to react with silanol intermediates (eqn (15)) leading to particle growth. These discrete, continuous kinetic events are clearly completely different than a burst nucleation and diffusion-controlled type of process. Moreover, there is excellent agreement among four different studies on the rate law (and its temperature dependence) of rate-determining $\mathrm{Si}(\mathrm{OEt})_{4}$ hydrolysis (as detailed on p. 199, Fig. 9 in Giesche's classic paper $^{205}$ ). That evidence indicates that $\mathrm{Si}(\mathrm{OEt})_{4}$ hydrolysis to $\mathrm{Si}-\mathrm{OH}$ containing species is kinetically continuous. However, it remains to be established if nucleation is continuous or not and what mechanism(s) and concepts are required to explain the strikingly narrow particle-size distributions seen in the
Stöber process. Zukoski's aggregative growth model is one idea that has promise, as discussed next.

6.1.2. Zukoski's aggregative growth model for the formation of silica and titania nanoparticles. In a series of important papers, Zukoski and his co-workers have reported support for an aggregation mechanism for the formation of uniform particles from the hydrolysis and condensation of silicon and titanium alkoxides. ${ }^{202,206-209}$ In the case of the hydrolysis and condensation of tetraethoxosilane (TEOS) in the presence of ammonia leading to the formation of nearuniform silica particles, ${ }^{210}$ Zukoski et al. report the following main findings: (i) the concentrations of TEOS and soluble silica measured, respectively, by ${ }^{29} \mathrm{Si}$ NMR spectroscopy and atomic absorption spectrophotometry after filtration undergo parallel changes at the same rate over the course of reaction, results that show that TEOS hydrolysis is the slow step of the reaction (as others also find ${ }^{204,205}$ ); (ii) the rate of TEOS consumption correlates well with the rate of particle growth; and that (iii) conductivity measurements are well fit by a A $\rightarrow$ B $\rightarrow$ C series reaction sequence with respective rate constants $k_{1}$ and $k_{2}$ (and where $\mathrm{A}=\mathrm{TEOS}, \mathrm{B}$ is hydrolyzed, conducting material, but $\mathrm{C}$ is less well defined) in which that the concentration of ionic, conducting species B passes through a maximum. The authors also report (iv) filtering the reacting solution (to remove the growing particles greater than $20 \mathrm{~nm}$ ) and then the observation that particle growth occurs in the filtrate solution; and that (v) seeded growth experiments show that the growth rate is independent of the particle number density.

Zukoski and co-workers then build a number density, population-balance model to try to explain the formation of near-monodisperse silica particles by (a) assuming an average nucleation rate " $\hat{g}_{n}$.",202,206 of formation of particles of size $r_{1}$ where ${ }^{206} g_{n}(t)=g_{s}\left[\mathrm{e}^{-k_{1} t}-\mathrm{e}^{-k_{2} t}\right]$ corresponding to the $\mathrm{A} \rightarrow \mathrm{B} \rightarrow$ $\mathrm{C}$ reaction sequence (and the form of $g_{\mathrm{s}}$ given on p. 353 elsewhere ${ }^{206}$ )-hence, continuous and not burst nucleation, plus (b) aggregative growth. Importantly, they find their model can (c) explain the narrow size-distribution as a result of aggregation (i.e., and not because of a "burst nucleation") in which the "primary particles aggregate with larger particles more rapidly than they do with themselves". ${ }^{202}$ The aggregative growth mechanism is further supported by the results of a 
cryoelectron microscope study ${ }^{211,212}$ revealing that the large final Stöber particles are not a single, dense particle but, instead, are actually compos of smaller, primary particles in loose, aggregated/polymeric structures ${ }^{209,212}$ as seen in Fig. S6 and S7 of the ESI. $\dagger$

However and unfortunately, the Zukoski aggregation model provides no explanation for the formation of uniform primary particles that then aggregate. A confusing statement in Zukoski's paper, that would make it appear as if burst nucleation is still believed and part of their model, is their statement on p. 33 in the 1991 paper $^{202}$ that their aggregation model “... is simply an extension of the 1950 model to the case where the nuclei may be colloidally unstable". Hence, an issue that remains with the Zukoski aggregation model is how the uniform particles that then aggregate are formed in the first place.

Worth mentioning here is that precedent has existed since $2005^{56-58}$ that the pseudo-elementary steps of B $+\mathrm{B} \rightarrow \mathrm{C}$ (rate constant $\left.k_{3}\right)^{55-58}$ then $\mathrm{B}+\mathrm{C} \rightarrow 1.5 \mathrm{C}\left(\text { rate constant } k_{4}\right)^{56-58}$ can quantitatively describe the kinetics of a number of aggregating systems, ${ }^{56-58,188,213-217}$ basically a sequence of bimolecular nucleation of aggregation $(\mathrm{B}+\mathrm{B} \rightarrow \mathrm{C})$ followed by smallerlarger size-focusing aggregation $(\mathrm{B}+\mathrm{C} \rightarrow 1.5 \mathrm{C})$ that has often been found to be faster, $k_{4} \gg k_{3} \cdot{ }^{56-58,188,213-217}$ Hence, further efforts are once again needed to understand what is a historically very important particle-formation system: namely how massive, nearly uniform size silica particles are formed.

6.1.3. Study claiming to observe a constant particlenumber density vs. time. A study by Okubo et al., ${ }^{218}$ of the formation of monodisperse silica nanospheres of $12 \mathrm{~nm}$ size in the emulsion system containing TEOS, water, and basic amino acids (and thereby weakly basic, $\mathrm{pH} 9-10$ conditions) provides evidence the authors believe is consistent with a LaMer-type model, specifically an apparently constant number density of silica particles as a function of time estimated by SAXS, Fig. 2 of that paper. ${ }^{218}$ However, the SAXS data are collected at times 0.5 , 1.0, 2.0, 3.0 and $4.0 \mathrm{~h}$, as shown in Fig. 2 of that paper, ${ }^{218}$ while ${ }^{1} \mathrm{H}$ NMR spectroscopic monitoring of the ethanol evolution in Fig. 3 of that paper ${ }^{218}$ shows $0.1 \mathrm{M}$ ethanol formation (corresponding to $7 \%$ TEOS consumption) at $0.5 \mathrm{~h}$ and then complete, $100 \%$ TEOS consumption to yield $1.37 \mathrm{M}$ ethanol at $12 \mathrm{~h}$. Critically, then, no SAXS data were collected in the first $0.5 \mathrm{~h}$ period during which $7 \%$ TEOS is hydrolyzed-and where considerable nucleation is likely taking place-and no SAXS data are collected in the last period after $4.0 \mathrm{~h}$ where $45 \%$ TEOS is consumed (i.e., where $55 \%$ of the TEOS remains unhydrolyzed). ${ }^{218}$ The FE-SEM images reveal the formation of silica nanoparticles $c a .4 \mathrm{~nm}$ in size in the aqueous phase at the early stage $(\sim 0.5 \mathrm{~h})$ of the reaction, ${ }^{218}$ again indicating that critical information on the key initial, nucleation stage of nanoparticle formation is hidden in this unobserved initial stage of the reaction. In short, the authors missed the early $(\sim 0.5 \mathrm{~h})$ stage and hence at least the initial nucleation, and missed the later $(4-12 \mathrm{~h})$ stage where aggregation is expected to dominate, so that the particle-number density versus time data they observe appears to be linear-and hence 1950-modellike-in the narrow range $0.5-4.0 \mathrm{~h}$ (one third of the whole reaction time, Fig. 2 of that paper $\left.{ }^{218}\right)$. Monitoring the reaction by ${ }^{1} \mathrm{H}$ NMR spectroscopy additionally shows a putatively linear release of ethanol, which could be interpreted as consistent with diffusion-controlled, constant rate growth.

However, the ostensibly zero-order kinetics for the hydrolysis of TEOS is inconsistent with the literature that invariably finds first-order [TEOS] $]^{1}$ hydrolysis kinetics ${ }^{204,205}$ (as for example shown in Fig. 10b elsewhere ${ }^{204}$ ). Furthermore, the ${ }^{29} \mathrm{Si} \mathrm{NMR}$ spectra taken from the solution after 0.5 and $3 \mathrm{~h}$ of the reaction are reported to show no signal for soluble silica species such as either silicate monomer or oligomers-negative evidence that is not usable without a demonstrated detection limit for those species. Note that the ${ }^{29} \mathrm{Si}$ NMR spectra were taken of the aqueous phase of the emulsion system with $[\mathrm{Si}]>1 \mathrm{M}$ conditions; ${ }^{218}$ and that silicon species in $\geq 1 \mathrm{mM}$ are expected to be readily detectable by ${ }^{29} \mathrm{Si}$ NMR. (The presence of detectable initial oligomers from "burst nucleation" is expected if the 1950 model is correct, so the ${ }^{29} \mathrm{Si}$ NMR spectroscopy would appear to be inconsistent with any model postulating burst nucleation.) Solid state ${ }^{29}$ Si MAS NMR spectrum of silica nanospheres after evaporation of solvent (Fig. 10 of that paper ${ }^{218}$ ) shows the existence of $\equiv \mathrm{Si}^{-} \mathrm{O}^{-}$or $\equiv \mathrm{Si}-\mathrm{OH}$ moieties in nanospheres, which are assumed to react with silicate monomers or oligomers when supplied at the interface of emulsion.

In short, the evidence for a constant number density of silica particles is equivocal, and (negative) evidence exists against any type of burst-formation of particles-the key point being that positive evidence will be required in order to support the author's claim of the 1950 model being applicable to their system. Moreover, the authors did not compellingly rule out Zukoski's competing mechanism of continuous nucleation followed by aggregative growth. Hence, the Okubo et al. system ${ }^{218}$ is another interesting system that merits further, clarifying examination.

6.1.4. deWith and co-Workers' 2014 study of the formation of silica nanoparticles. A 2014 paper by de With et al. ${ }^{219}$ reports the formation of near monodisperse amorphous silica nanoparticles of final $24.0 \pm 2.8 \mathrm{~nm}$. Primary nanoparticles of $1.3 \pm$ $0.3 \mathrm{~nm}$ after $24 \mathrm{~h}$ reaction are formed in this system and from the same procedure described by Okubo et al. ${ }^{218}$ Some important insights from this paper ${ }^{219}$ relevant to this review are: (i) large particles are again shown to be composed of aggregated smaller, primary nanoparticles; (ii) the primary small nanoparticles are colloidally stabilized by charged silanolate groups on the surface; (iii) those silanolate groups enable particle association by cross-linking; (iv) the formation of large particles from primary particles is proposed to be "the association of primary particles together with their further collapse"; (v) aggregative growth stops when no more primary small nanoparticles are present; and (vi) association and aggregative growth occur concomitantly with an increase in cross-linking. The main point relevant to this review is that these insights can be explained by Zukoski's model ${ }^{202}$ of assumed continuous nucleation plus aggregative growth, but cannot be explained by the 1950 model. ${ }^{1} \mathrm{~A}$ second point here is that these authors apparently do not recognize (and did not cite 
in their paper ${ }^{219}$ ) that instead of a word-only description of the reaction as cited above, the pseudo-elementary steps of bimolecular nucleation of agglomeration $(\mathrm{B}+\mathrm{B} \rightarrow \mathrm{C})$ and autocatalytic agglomeration $(\mathrm{B}+\mathrm{C} \rightarrow 1.5 \mathrm{C})$ were available $^{55-58}$ at the time of their 2014 paper and could have been used to try to describe their particle formation system. Hence, kinetics data should have been collected and fits to the data by the above, 2-step agglomeration mechanism would then have provided further support for, or refutation of, their proposed reaction pathway. The truism that there is no mechanism without kinetics is apparent once again.

\subsection{Iron-oxide nanoparticles obtained by thermal decomposition of precursor}

6.2.1. Claims for an "Extended LaMer Mechanism" for the formation of magnetite nanoparticles. Huber et al. have proposed an "Extended LaMer mechanism" for the synthesis of magnetite nanoparticles in a system that involves the continuous, constant rate, syringe-pump feeding of iron(III) oleate precursor to the $350{ }^{\circ} \mathrm{C}$ reaction solution. ${ }^{220}$ Noteworthy positives of this paper include (i) the monitoring of the iron(III) oleate precursor and the other efforts aimed at providing as reproducible a synthesis as possible, and that (ii) the control of particle size is achieved simply by varying the reaction time (i.e., the amount of added precursor from the syringe-pump feed). By comparison, the classical synthesis of iron-oxide nanoparticles is more complex and starts with a solution of ferric salt at $\mathrm{pH}$ 0.5-2.5, ages it at room temperature for a certain time, then heats the solution in a Teflon-lined screw cap Pyrex culture tube to $c a .100{ }^{\circ} \mathrm{C}$, and finally, cools the reaction to room temperature. ${ }^{221}$

The continuous-precursor-feeding synthesis was used by Huber et $a .^{220}$ for the preparation of crystalline magnetite $\left(\mathrm{Fe}_{3} \mathrm{O}_{4}\right)$ nanoparticles of sub-nanometer size of low size dispersity, between $9.3 \pm 0.7 \mathrm{~nm}$ and $34.5 \pm 1.6 \mathrm{~nm}$. The Fe(III) oleate precursor was prepared from the reaction of $\mathrm{Fe}(\mathrm{acac})_{3}$ and excess oleic acid (solvent) at $320{ }^{\circ} \mathrm{C}$ and used without isolation or purification. The Fe(III) oleate in oleic acid plus 3.0 equiv. acetylacetone per mole of iron was diluted in 1-octadecene and then dripped a constant rate of $3 \mathrm{~mL} \mathrm{~h} \mathrm{~h}^{-1}$ using a syringe pump into the reaction flask containing docosane and oleic acid and then heated to $350{ }^{\circ} \mathrm{C}$. The precise balanced stoichiometry of the reaction was not established but is of interest, as that experimental stoichiometry is the necessary first step en route to understanding the underlying mechanism of formation of magnetite nanoparticles with such a narrow size distribution. Recall that as detailed elsewhere, ${ }^{2}$ a balanced reaction stoichiometry is a necessary first step of any reliable mechanism determination because the steps of the proposed mechanism must add up to (and thus serve as a check on) the observed, balanced reaction stoichiometry. Otherwise, one is proposing a mechanism for some reaction other rather than the one under study!

The diameter of magnetite nanoparticles versus time data, obtained by SAXS measurements and redisplayed in Fig. 24, was obtained as part of the reported studies. ${ }^{220}$ The authors attempted to interpret the growth of the nanoparticles by using a (words only, vide infra) combination of the burst-nucleation model and the FW 2-step mechanism-which at the outset makes no sense, given that the two are at the opposite ends of the spectrum of the kinetics of particle formation ("instantaneous/burst nucleation" and "diffusion-controlled growth"1 being the basis for the 1950 model, while the 2-step mechanism involves "slow, continuous nucleation", and slower than diffusion controlled, "chemical reaction-controlled autocatalytic surface growth", $)$. Unfortunately, the authors did not fit their data with either model and, instead, used an empirical power function unconnected to either model/mechanism, all while ignoring the first 50 minutes of data (Fig. 24) in the fitting, the time during which significant nucleation is expected to occur. $^{220}$

The authors attempted to rationalize their results using a word-only, blended "LaMer plus FW-2-step" model they called the "Extended LaMer mechanism", illustrated in Fig. 25. ${ }^{220}$
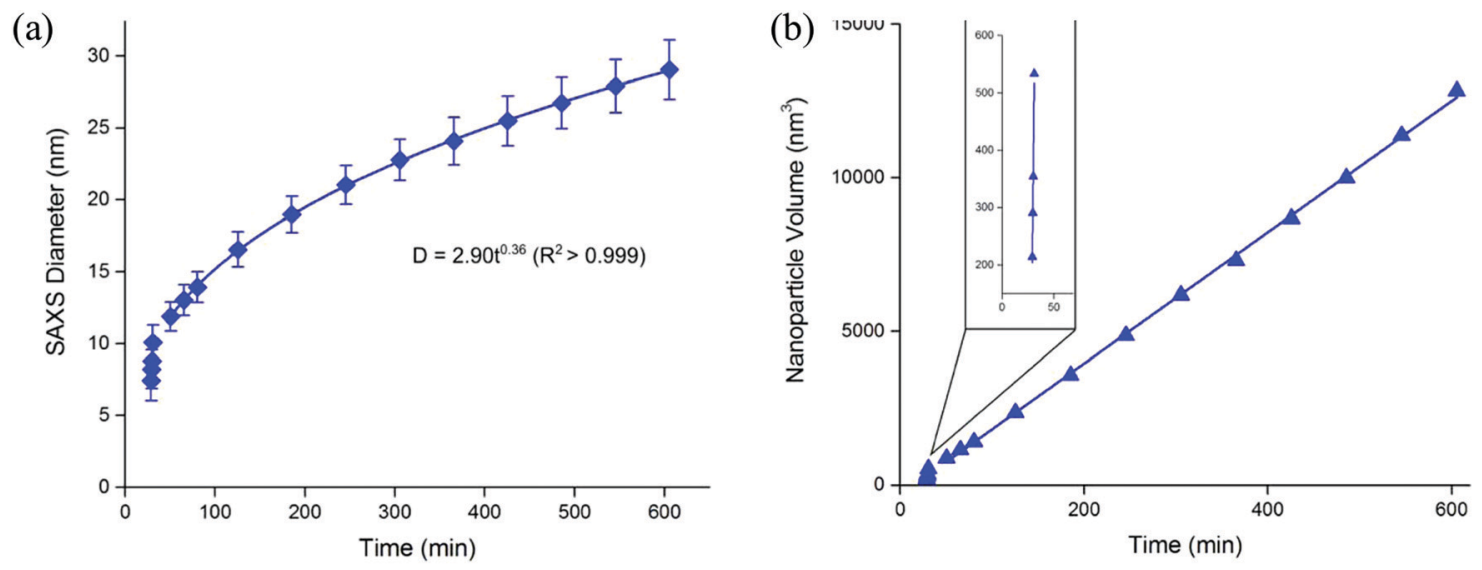

Fig. 24 (Fig. 3 in ref. 220.) (a) Plot of diameter versus time calculated from SAXS measurements of the aliquots of magnetite nanoparticles during the decomposition of iron(III) oleate at $350{ }^{\circ} \mathrm{C}$ in docosane solution plus oleic acid and 3.0 equiv. acetylacetone at a feeding rate of $3 \mathrm{~mL} \mathrm{~h}^{-1}$. (b) Plot of nanoparticle volume against reaction time with a close-up of the first four aliquots measured shown in the inset. Reproduced with permission from ref. 220. Copyright 2015 American Chemical Society. 


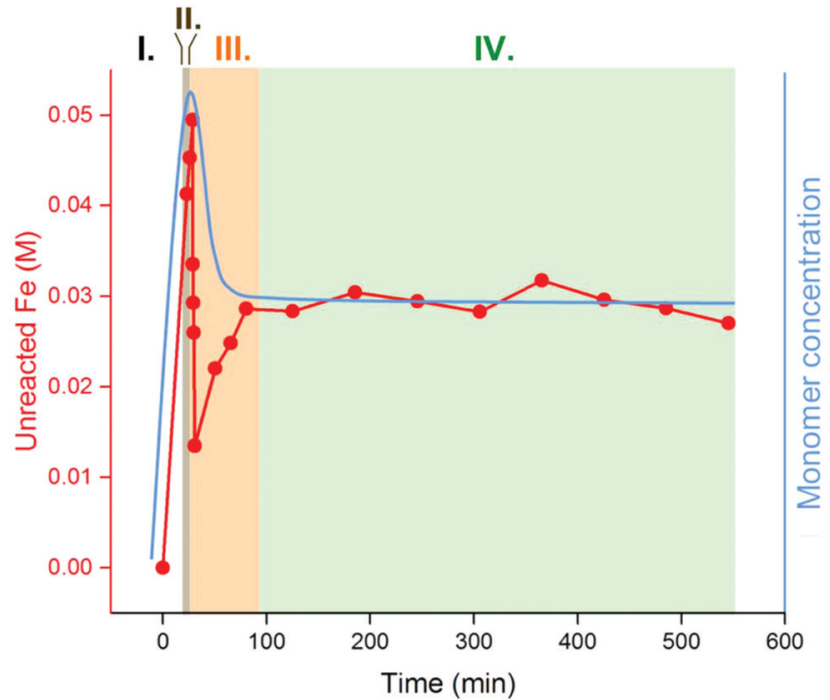

Fig. 25 (Fig. 5 in ref. 220.) Experimental data of the unreacted iron precursor (red) are overlaid by a cartoon schematic representing an "extended LaMer" approach to nanoparticle synthesis (blue). The presumed four stages of the postulated "Extended LaMer mechanism" are shaded to identify their believed and hence imposed, approximate boundaries. Reproduced with permission from ref. 220. Copyright 2015 American Chemical Society.

An examination of Fig. 25 reveals that it superimposes the experimentally determined iron(III) concentration present in solution $^{220}$ (left-most $y$ axis) with a cartoon presentation of the hypothetical soluble (1950-model-type) monomer concentration ${ }^{1}$ on the right-most $y$ axis.

The problem with Fig. 25 is that the concentration of iron(III) oleate present in solution cannot be the same as (and therefore is misleading to co-plot along with) the concentration of the hypothetical soluble monomer of magnetite, $\left(\mathrm{Fe}_{3} \mathrm{O}_{4}\right)_{n}$, whatever that may be. ${ }^{222}$ It at least appears that the authors have made the intellectual mistake of trying to "prove a mechanism" while actually constructing a single model that is an ill-advised combination of two physically opposite models. What is needed instead is proper scientific method of attempting to disprove the models, ${ }^{223,224}$ so that any that cannot be disproved at present remain as possibilities for further, future testing. The failure to try to quantitatively fit the data by either model, while instead using a "words-only" approach, is another classic mistake in the attempted application of physical models as detailed elsewhere. ${ }^{2}$ Additionally, a chemical reaction mechanism requires balanced chemical reactions that define the rate constants, the associated differential equation, and also quite importantly defines the precise words one can use to describe the proposed, stepwise processes, none of which are present as part of this work. ${ }^{220}$

Returning to Fig. 25, this figure has little to do with a LaMertype model, as the four I-IV "phases" labeled in Fig. 25 in an attempt to support a LaMer-type model are, instead, very likely just (I) the initial rise in the precursor expected from injecting it; (II) nucleation and likely autocatalytic growth, perhaps following the 2 -step mechanism ${ }^{3}$ as noted by the authors; ${ }^{220}$
(III) a dip below steady-state for reasons that are unclear at present (perhaps due to smaller particles reacting faster than larger ones ${ }^{61,62}$ ); and (IV) then steady-state in the continuous injection system. Most telling in this work is how misleading it is to stick with one's initial hypothesis (anchor bias), in this case the hypothetical "Extended LaMer mechanism", and then perform confirmation bias from one's anchor bias starting point by gathering only the data consistent with one's single, starting hypothesis. Application instead of Chamberlin's ${ }^{223}$ and Platt's ${ }^{24}$ superior scientific method emphasizing ${ }^{225}$ disproof of multiple alternative hypotheses-especially attempting to disprove one's own original, pet hypothesis-is once again what was needed here. As Platt notes "for exploring the unknown, there is no faster method" 224 than the attempted disproof of multiple, alternative hypotheses.

Nevertheless, the clever use of the open, continuous injection of precursor system is noteworthy, ${ }^{220}$ even if not new in studies that mention the 1950 model $^{99-101,104,220,226,227}$ (e.g., Sugimoto's 1992 report on the formation of colloidal silver bromide in an open system where two solutions of silver nitrate and potassium bromide were simultaneously and continuously introduced into an aqueous solution of inert gelatin at a controlled rate, ${ }^{100}$ a study we covered back in Section 3.2). The attention to a reproducible formation of the iron oleate precursor, and to a reproducible synthesis, are other valuable parts of this study ${ }^{20}$ that merit further examination and attempts to quantitatively understand the underlying mechanism and advantages/disadvantages of the open, continuous precursor-injection system(s). Any more detailed look at this system should also endeavor to understand the "question of what causes the batch-to-batch variation in particle sizes" identified by the authors via their careful experimental work (see p. 60603). ${ }^{20}$ Possible explanations meriting attempted disproof include (a) higher kinetic order (i.e., concentration) effects on nucleation, ${ }^{17,26,27}$ (b) Epstein's important work on the large effects of imperfect mixing on reactions involving autocatalytic steps, ${ }^{228}$ and (c) the effects of dust (see Section 10).

6.2.2. Formation of iron-oxide nanoparticles via precursor thermal decomposition. Synthesis of iron-oxide nanoparticles with sizes in the range $4-28 \mathrm{~nm}$ has been achieved by thermal decomposition of iron(II) stearate in the presence of 2 equivalents of oleic acid and in refluxing octyl ether solvent. ${ }^{229}$ The TEM-determined particle diameter as a function of time is reproduced in Fig. 26a. ${ }^{229}$ Somewhat large error bars are observed, although it is not clear if those reflect the underlying chemistry or some other effect(s). ${ }^{230,231}$ The 1950 model was used to rationalize the nanoparticle formation, albeit in a words-only way without supporting evidence.

The authors conducted in-depth characterization of the resultant nanoparticles, including extensive magnetism studies, that are noteworthy. However, the particle formation was attributed to the 1950 model using a words-only, qualitative explanation $^{229}$ of how the synthetic parameters are claimed to influence their reaction via the 1950 model. Presently, no data support, or definitively refute, the 1950 model for this iron-oxide system. What is needed to move this system to a 


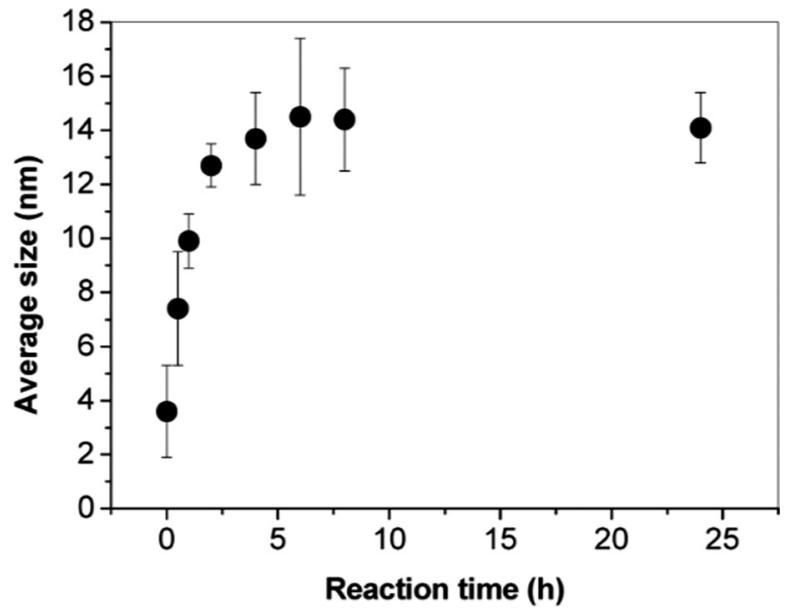

Fig. 26 (Fig. 1a in ref. 229.) Average diameter of iron-oxide nanoparticles as function reflux time. The nanoparticles are formed from the decomposition of iron(II) stearate in the presence of 2 equivalents of oleic acid (stabilizer) and refluxing in octyl ether (solvent, b.p. $287^{\circ} \mathrm{C}$ ). Reproduced with permission from ref. 229. Copyright 2014 American Chemical Society.

better understood level is the complete, balanced reaction stoichiometry and then kinetics and mechanism-based differential equations to describe the pseudo-elementary steps that lead to iron-oxide formation.

A 2008 account by Kwon and Hyeon ${ }^{232}$ reports the formation of iron-oxide nanocrystals from thermal decomposition of $\mathrm{Fe}(\mathrm{CO})_{5}$ with oleic acid again in dioctyl ether. The authors claim that the nanoparticle formation occurs in three steps consistent with the 1950 model: accumulation of iron(III) oleate monomer, burst nucleation, and size focusing. However, their kinetics data for the extent of the thermal decomposition reaction and concomitant formation of iron-oxide nanocrystals (Fig. 15 of ref. 232) show an induction period in an overall sigmoidal curve, inconsistent with putative "burst nucleation", but suggestive instead of continuous nucleation. Hence, the evidence in this 2008 study also argues for the inapplicability of the burst-nucleation model to the formation of iron-oxide nanoparticles.

In short, there is no compelling evidence for, and suggestive evidence against, the burst-nucleation model's applicability to iron-oxide particles as well as to silica particles. Some evidence that these systems actually involved continuous nucleation is apparent, arguing that more detailed kinetics and mechanistic investigations are needed in order to be able to write down the true particle-formation mechanism for these metal-oxide systems.

\subsection{Formation of other metal-oxide nanoparticles}

Thirty-nine other papers on metal oxide nanoparticles that cite the 1950 model have been analyzed as summarized in Table S4 in the $\mathrm{ESI} \dagger$ for the interested reader. In addition to the papers that have been discussed (vide supra), the following are included in Table S4 (ESI $\dagger$ ): cobalt oxide, ${ }^{233}$ copper oxide, ${ }^{234,235}$ zirconia, ${ }^{236}$ silica and titania, ${ }^{104,237-243}$ iron oxide ${ }^{244-249}$ indium oxide, ${ }^{250}$ and a mathematical model. ${ }^{251}$ In all of these reports save three exceptions, ${ }^{233,237,238}$ the 1950 model is used to rationalize the observations, albeit in a "words-only" fashion. Hence, in each of these cases, additional studies are needed before a better understanding of their particle formation mechanism(s) can be claimed.

\section{A reminder about the 1950 model's quantitative equations-and the disconnect versus the words-only "burst-nucleation" and diffusion- controlled growth qualitative model}

Noteworthy is that none of the studies examined to this point, nor any others ${ }^{2}$ of the nearly two-thousand papers citing the 1950 paper, ${ }^{1}$ successfully use the 1950 paper's quantitative radius $^{2}\left(x^{2}\right)$ equation to fit their data, eqn (17). Nor has any paper or study citing the burst-nucleation model used the "special case" as it is called in the 1950 paper, ${ }^{1}$ eqn (18), to fit their data. Noteworthy here is that eqn (17) is the equation LaMer used to fit his smoothly increasing, downwardly concave $(\mathrm{S})_{n}$ sol radius vs. time data in Fig. 7 of his 1950 paper $^{1}$-data that does not show any "burst" process. ${ }^{1}$

$$
\begin{gathered}
\frac{\mathrm{d}}{\mathrm{d} t}\left(x^{2}\right)=\left[C_{\mathrm{ss}}-C_{\mathrm{s}}(t)\right] \frac{2 D}{\rho}-\frac{2 D}{h^{3}} x^{3} \\
t=\frac{h^{2}}{3 D}\left(\frac{\rho}{C_{0}}\right)^{1 / 3}\left\{\frac{1}{2} \ln \frac{\left[\left(\frac{C_{0}}{\rho}\right)^{2 / 3} h^{2}+\left(\frac{C_{0}}{\rho}\right)^{1 / 3} h x+x^{2}\right]}{\left(x-\left|\frac{C_{0}}{\rho}\right|^{1 / 3} h\right)^{2}}\right. \\
\left.+\sqrt{3}\left[\tan \frac{-1 \sqrt{3}}{3}-\tan ^{-1}\left(\frac{2 x+\left|\frac{C_{0}}{\rho}\right|^{1 / 3} h}{\left|\frac{C_{0}}{\rho}\right|^{1 / 3} h \sqrt{3}}\right)\right]\right\}
\end{gathered}
$$

Highly relevant here is the little-discussed, extreme assumption made in the 1950 paper that (i) to get to eqn (18), $C_{\mathrm{s}}(t)$ in eqn (17) was assumed to be constant and not time dependent as it physically is in order to be able to integrate eqn (17) to obtain eqn (18). This is of course an extreme assumption because eqn (17) teaches that the time-dependent $C_{\mathrm{s}}(t)$ helps inform the time dependence of $x^{2}$. Also little appreciated nor previously discussed to our knowledge is the point alluded to just above, specifically that (ii) the particle radius $v s$. time data in Fig. 7 of the 1950 paper $^{1}$ and fit by eqn (17) do not contain any type of burst feature. That is, (iii) there is a serious disconnect between the actual, finally employed mathematical model in eqn (17) and the highly cited, words-only, qualitative diagram that is Fig. 1 in the 1950 paper that is reproduced herein as the earlier in Fig. 7a. This disconnect means in turn (iv) that $\sim 90 \%$ ( $\sim 1761$ papers) of the papers that cite the burst-nucleation qualitative model for just the burstnucleation feature are either ignoring, or are unaware of, the fact that the model's quantitative equation used in the classic 
1950 paper to fit data shows no burst feature! This relates to a point made in our Part 1 review: ${ }^{2}$ the "burst nucleation" model is really a growth model that postulates/assumes "instantaneous" nucleation out of necessity in order to derive the $\mathrm{d}\left(x^{2}\right) / \mathrm{d} t$ eqn (17) that is a mathematical model for particle growth. ${ }^{2}$

\subsection{Recent demonstration of a "burst-nucleation-like plot"-that actually stems from continuous nucleation and size-dependent growth}

Of interest here is that the new 3-step mechanism discovered recently via mechanism-enabled population-balance modeling (ME-PBM), for the $\operatorname{Ir}(0)_{n}$ nanoparticle formation, ${ }^{61,62}$ yields a nucleation plot for the formation of the experimentally established $\operatorname{Ir}_{3}$ nuclei that looks a lot like it might support a "burstnucleation-type" mechanism. See especially the top middle plot of the nucleation rate $v s$. time in Fig. 27 below. Most researchers looking at that nucleation rate $v s$. time plot would probably believe that it supports the classic 1950 model and associated, highly cited qualitative figure back in Fig. 7a.

However, the top middle plot of the nucleation rate comes (as do the other plots in Fig. 27) from the ME-PBM with the 3 -step mechanism of kinetically continuous nucleation ${ }^{3}$ (specifically experimentally determined continuous "alternative termolecular" nucleation ${ }^{26}$ ) that can be summarized in a minimalistic way by the pseudo-elementary step of $\mathrm{A} \rightarrow \mathrm{B}$ (rate constant $\left.k_{1}\right)^{17,26,27}$ and then the size-dependent growth steps of $\mathrm{A}+\mathrm{B} \rightarrow \mathrm{C}$ (rate constant $k_{2}$ ), and $\mathrm{A}+\mathrm{C} \rightarrow 1.5 \mathrm{C}$ (rate constant $\left.k_{3}\right){ }^{61,62}$ the last mechanistic model back in Table 2 . The rates for both nucleation and growth appear to spike and be "burstlike", yet these rates are actually the result of kinetically continuous nucleation. The full details are available elsewhere for the interested reader. ${ }^{62}$

The perhaps obvious take-home message is that a curve that qualitative looks like "burst nucleation" may actually really be due to burst-like autocatalytic growth, a feature that will deceive the unwary researcher not using a proper, disproof-based scientific method. ${ }^{223,224}$ Researchers not also using quantitative equations from either mechanisms or other models to try to fit the kinetics and information-rich $\operatorname{PSD}^{61,62}$ data also have a strong chance of being deceived into claiming they have observed "burst nucleation" as assumed in the 1950 mathematical model.

\section{Alternative literature models/ mechanisms that cite the 1950 model}

The following topics are summarized in seventeen entries in Table S5 in the ESI $\dagger$ for the interested reader: kinetics of precipitation from supersaturated solid solutions, ${ }^{252}$ oriented attachment at crystallographically specific surfaces, ${ }^{253-255}$
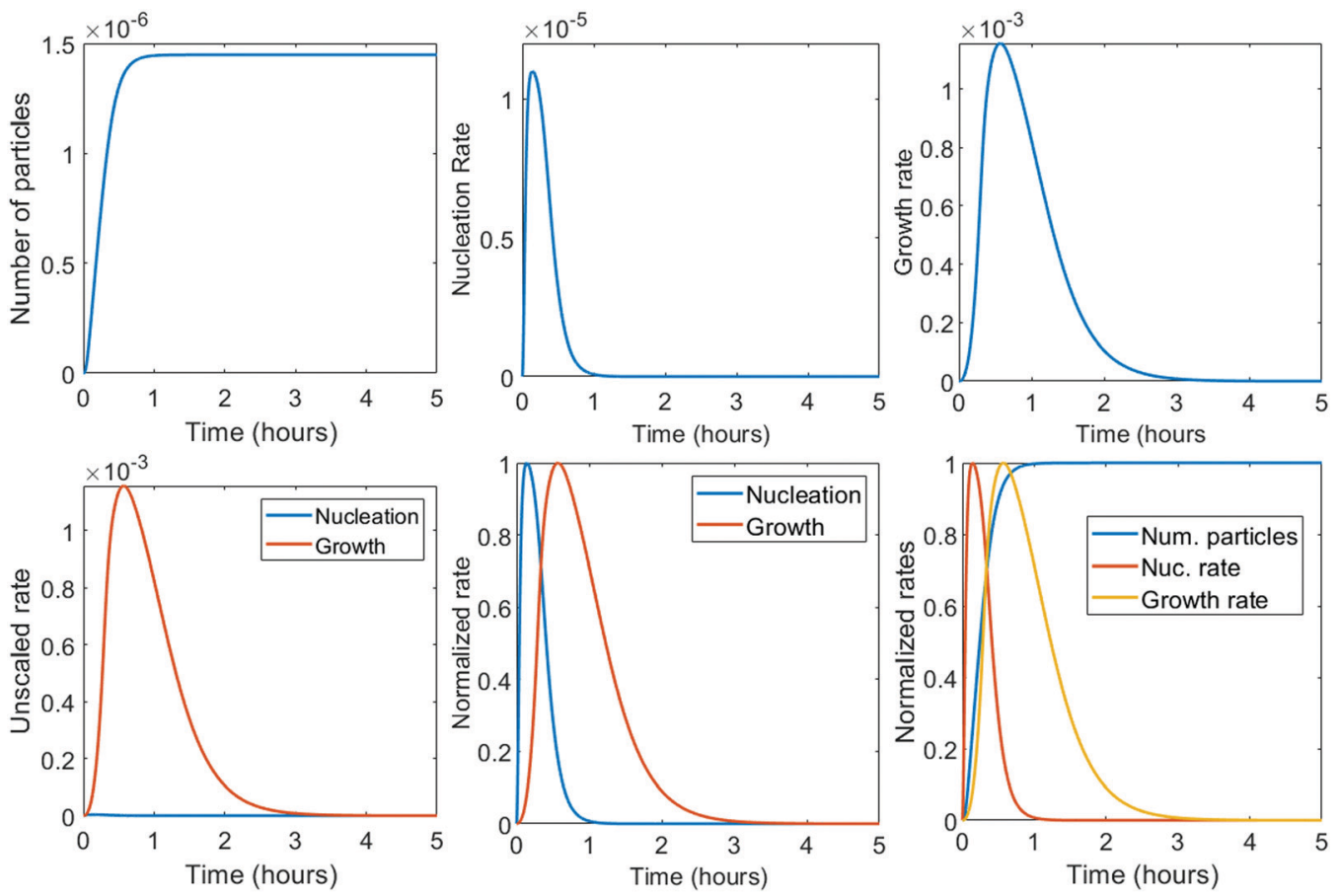

Fig. 27 Numerically calculated number of particles $(M)$, nucleation rate $\left(\mathrm{M} \mathrm{h}^{-1}\right)$, and growth rate $\left(\mathrm{M} \mathrm{h}^{-1}\right)$ for the 3-step mechanism with the experimentally established nucleation mechanism ("alternative termolecular nucleation"26,61,62) used to construct the mechanism-enabled population balance model. Both unscaled (top) and scaled (bottom) versions of the results from the ME-PBM are shown. The number of particles increases monotonically demonstrating the continuous nucleation of particles. The nucleation rate spikes, and appears "burst-like", but actually involves kinetically continuous nucleation. The burst feature is actually due to burst-like autocatalytic growth, as shown in the bottom-left figure. The spike periods of nucleation and growth where the two rates are both relatively very high overlap for approximately one fifth of the measured time. Reproduced with permission from ref. 62. Copyright 2020 American Chemical Society. 
inverting the nucleation and growth processes, ${ }^{256,257}$ aggregative growth processes, ${ }^{258-265}$ another three stage mechanism, ${ }^{251}$ rethermalization and irreversible diffusional growth, ${ }^{266}$ mechanisms of diffusional nucleation of nanocrystals and their self-assembly into uniform colloids, ${ }^{267}$ a classical model and a different two-step model for nucleation of crystals from solution, ${ }^{16}$ nucleation and growth of nanoparticles in the atmosphere, ${ }^{12}$ dispersive kinetics $^{268}$ nanoscale-modified LaMer model, ${ }^{269}$ Burst-Schiffrin methods and associated population-balance modeling, ${ }^{270}$ and the FW 2-step mechanism extended to a photomechanical 9-methylanthracene crystal system. ${ }^{271}$ We refer the interested reader to those studies and Table S5 (ESI $\dagger$ ).

\section{Conclusions}

This second, Part II review has covered the Classical Nucleation and Fluctuation Theory basis for the 1950 model and the models and main pseudo-elementary step, disproof-based mechanisms for phase changes and particle formation in the literature since the 1950 paper by LaMer. Specifically, the papers in the literature up to March 2019 were presented that cite the burst-nucleation model while also providing data for silver halide, semiconductor, metal, and metal-oxide particle formation systems. Those studies contain a wealth of data from often cleverly designed and many times expertly executed studies, more recently using powerful synchrotron-based XAFS and SAXS methods. The primary conclusions one can draw are the following, while emphasizing that we and the community have the advantage of $c a .70$ years of additional knowledge, advanced experimental and computational methods and hence associated hindsight in reaching the following conclusions compared to the information and tools that were available in 1950.

- Classical nucleation theory that underlies the original burst-nucleation model $^{1}$ was designed for weakly interacting systems where the identity of the associating unit is retained, such as hydrocarbon association in the gas phase, and where supersaturation is not greatly exceeded. CNT has proved inapplicable to strong bonding systems far beyond supersaturation such as arguably all of the systems and papers examined in this review and the tables in the ESI. $\dagger$

- No study other than the original 1950 paper $^{1}$ fits experimental particle radius versus time data with the 1950 paper's equation, eqn (17) vide supra. Noteworthy is that the Fig. 7 data in the 1950 paper that is fit with eqn (17) does not contain a burst nucleation feature. One attempted fit by others using eqn (17) for a $(S)_{n}$ formation system failed ${ }^{272}$ as discussed in our Part I review. $^{2}$

- Clear, convincing, definitive experimental evidence for either "instantaneous/burst" nucleation or "diffusioncontrolled" growth is not apparent in any of the nearly twothousand papers examined that cite the 1950 model to date, at least so far as we have been able to discern. Even for the previously believed "best examples" of $(\mathrm{AgX})_{n}$ formation or hotinjection methods for making semi-conductor nanoparticles, compelling kinetics evidence for anything approaching "instantaneous" or burst nucleation seems to be lacking. Our apologies in advance to the Community if we have somehow overlooked a key paper or papers containing definitive evidence as we scoured through the voluminous literature citing the 1950 model.

- A number of studies conclude that their evidence disproves "instantaneous/burst" nucleation in those different, specific systems. Several of those specific systems and studies were mentioned in the main text. ${ }^{3,5,6,17,26,27,48,51,60-63 \text {, }}$ $71,121,122,130,132,159,164,170,188,189,206,233,237,238,260,261,271$ All of those studies, and others ${ }^{273-275}$ that disprove the model, can be found in the tables provided as ESI $\dagger$ and are labeled as providing evidence disproving the 1950 model for their system.

- The 32 independent papers cited just above, when combined with the lack of evidence uncovered from a comprehensive look at the papers citing the 1950 model, provides strong, seemingly compelling evidence against the 1950 mathematical model as well as the often-cited, words only version of the model in the 1950 paper. The reader who has taken time to study the 74 pages and 5 tables in the ESI $\dagger$ can compare this statement to their own conclusions. Note here that we do not mean to imply that words-only models are not useful in science-indeed, qualitative word-only statements are the most powerful in science, but they often come out of, or at least are strongly and best supported by, quantitative data. But for the words-only version of LaMer's 1950 model, one can now state with some confidence that words-only version has not proved useful-and has also proved difficult to try to disprove, thereby hindering scientific advance. Additional discussion of and references to words-only $v s$. mathematical $v s$. mechanistic models in science is contained in our Part I review (e.g., see Section 2.6 there $^{2}$ ) as well as elsewhere ${ }^{51}$ for the interested reader.

- Numerous studies that claim their system undergoes "instantaneous/burst" nucleation actually contain data that in our opinion disproves that claim. ${ }^{99,107,108,147,165,169,220,256}$

- At least at present the widely cited and often reproduced "LaMer figure" (Fig. 1 in the 1950 paper, ${ }^{1}$ Fig. 1a in Part I of this series, ${ }^{2}$ and reproduced herein as Fig. 7a, vide supra) remains to be experimentally verified and, hence, constructed from actual, quantitative experimental data rather than its status currently as an interesting artistic drawing.

- The valuable Sugimoto et al. study of $(\mathrm{AgX})_{n}$ formation and its associated Fig. 8 merits additional studies, plus efforts to understand mechanistically the spike in the $\left[\mathrm{Ag}^{+}\right]$supersaturation ratio seen in Fig. 8 of that classic study-is that spike really a spike in growth rather than nucleation, is one key question that needs to be addressed.

- At present and based on the available literature, one if forced to conclude that the creative 1950 model has little experimental support even after 70 years and despite many claims to the contrary.

- One cannot escape contemplating what would have expedited moving the science and our understanding of nucleation and growth beyond the 1950 model at a faster pace? 
Some thoughts are summarized in a footnote for the interested reader, ${ }^{276}$ including an excellent, recent ACS Catalysis editorial entitled "The Burden of Disproof",225 and thoughts about a better consideration and understanding of the different types of models in science. ${ }^{277-279}$

- Table 2 summarized the main pseudo-elementary step, disproof-based mechanisms for phase changes and particle formation that have appeared in the literature since the 1950 paper and that are in more general "A, B, and C..." species form. Those mechanistic models are available for fitting and initial interpretation of often sigmoidal-shaped particle formation and other phase-change kinetics data, as well as for mechanistically enabling population balance models.

- The available evidence across the systems studied and reviewed herein, and hence a primary working hypothesis going forward to test and to try to disprove, is that nucleation may well more often be continuous ${ }^{3}$ kinetically.

- A very important point is that kinetically continuous nucleation, even when slower than explosive, exponential, autocatalytic growth as may often be the case, can yield a "burst" of nucleation and can show some separation of nucleation and growth in time, as demonstrated in Fig. 27. What is most often probably observable and hence observed is not burst nucleation, but instead burst growth to first observable clusters $^{17}$ according to the sensitivity of whatever physical method is being employed. A separate publication is available expanding on the idea of "burst nucleation $v s$. burst growth",280 while also addressing a few additional issues about the 1950 diagram revealed during the construction of the present review.

- Another important point is the finding that a 3-step mechanism, that is a 1-step upgrade of the 2-step mechanism that can allow smaller particles to grow faster than larger particles, can give rise to narrow PSDs despite continuous nucleation.

- However, the remarkable fact that narrow dispersions of particles are known to result even after tens of thousands (to millions) of assembled atoms or structural units in cases such as silica particles - that, therefore, must have an equivalently large number of elementary steps in their actual, atomistic mechanistic steps-indicates that novel, to-be-discovered mechanistic insights remain hidden in such massive self-assembly systems.

- The need for additional in operando experimental measurements able to time resolve especially nucleation but also growth (as well as any agglomeration), ideally by multiple, complementary physical methods, is apparent and a conclusion reached at the end of several different sections in the review. Fortunately, XAFS, SAXS, in situ microscopy and other advanced physical method studies of multiple systems by a variety of expert investigators are currently ongoing and available to the community.

\section{A look towards the future}

The future for a better understanding of particle and crystal formation across nature is actually bright in our view. First, a large number of talented researchers using the most powerful physical methods such as SAXS, XAFS, environmental EM, and other method are investigating a variety of nucleating, growing and often agglomerating systems across nature, often in situ or in operando. A number of models and mechanistic efforts were cited in the main text and are noted in the tables in the ESI $\dagger$ that are disproof based and merit additional, careful and deeper investigation along with further attempted disproof anywhere their application might make sense. Disproofbased, deliberately minimalistic 2-, 3-, and two 4-step models that include two types of aggregation/agglomeration are available for fitting kinetics data involving nucleation, growth, and agglomeration processes - with the caveat that attention needs to be paid to the well-advertised limitations of those deliberately minimalistic, Ockham's razor-obeying mechanistic models. ${ }^{48,60,89}$ Detailed, molecular-level insights into nucleation for strongly bonded systems ${ }^{17}$ are beginning to appear, ${ }^{17,26,27}$ and the involvement of dust and prevalence of heterogeneous nucleation ${ }^{5,281-298}$ are becoming re-appreciated-noteworthy here is that the effects of dust on water vapor nucleation have been known since seminal studies the late 1800s. ${ }^{281-286}$ The concept of prenucleation clusters is gaining experimental support and hence momentum $^{72-76,175-178}$ - modern ideas that connect back to Turkevich's original “Organizer" concept. Ligand effects are obviously very prevalent in particle formation (e.g., in the studies of semi-conductor particle formation, Table S2 of the ESI $\dagger$ ), and just now being added to minimalistic mechanisms in both the nucleation step from an A.L precursor $(\mathrm{A} \cdot \mathrm{L} \rightleftharpoons \mathrm{A}+\mathrm{L})^{26,27,63}$ and subsequent, pseudo-elementary ${ }^{3}$ steps, notably ligand (L)-binding to the nanoparticle product $(\mathrm{B}+\mathrm{L} \rightleftharpoons \mathrm{B} \cdot \mathrm{L})$, as demonstrated in A. Karim's insightful studies of $\mathrm{Pd}_{n}$ nanoparticle formation that involve both A.L and B.L species. ${ }^{63,192}$ Considerable more effort to document, understand, and exploit ligand effects, and to elucidate their underlying mechanistic basis, seem well warranted if not wise. The combination of the precursor $^{26,27,63} \mathrm{~A} \cdot \mathrm{L}$ and product B.L equilibria, ${ }^{63}$ with the $2-, 3-$, and two 4-step minimum mechanisms now available,,$^{3,17,55-58,60,187,188}$ along with minimal unimolecular ${ }^{3}$ and precedented unimolecular (but in a bimetallic precursor), ${ }^{27}$ second-order, ${ }^{17}$ and ter-molecular (specifically "alternative termolecular",26) nucleation, one can write out 5 subclasses of mechanisms containing 96 reasonable, distinct mechanisms to try out for one's particle formation (and any subsequent agglomeration) system. ${ }^{61,62}$ The anticipated result is the desired synthetic control over especially particle size, particle-size dispersity, and surface ligands that a mechanism-based understanding promises to give. Computational studies continue to become more and more relevant and of increasing reliability. ${ }^{36,37}$ One can also examine other models/theories for particle growth as alternative hypotheses for the system at hand and to contrast a mechanism-based approach if desired, Table 1, vide supra. Analysis and insights from other areas where nucleation and growth are involved and actively studied such as protein aggregation are available for study. ${ }^{64}$

Promising and potentially very powerful is the advent of mechanism-enabled population balance modeling (ME-PBM) 
as a tool for predicting particle-size distributions (PSDs) from a given mechanism, as well as for use in the "inverse problem" of extracting the kinetics data buried within the convolutedinformation-rich PSD to inform and refine the particle formation mechanism. ${ }^{61,62}$ One now can and should test one's proposed particle formation mechanism with the powerful tool of ME-PBM. ${ }^{61,62}$ Especially important is the paradigm-shifting finding from that ME-PBM that narrow size distributions do not require undemonstrated "instantaneous/burst nucleation". Instead, narrow PSDs can result from continuous nucleation because smaller particles can grow faster than larger ones, thereby catching up with the larger particles en route to narrow PSDs. ${ }^{61,62}$ Zukoski's PBM work on aggregating systems emphasizing that "primary particles aggregate with larger particles more rapidly than they do with themselves"202 is important, noteworthy, early work that is likely to have broader impact. Polte's scholarly review ${ }^{186}$ discusses the "Influence of coalescence kinetics on particle size" and emphasizes the somewhat different concepts of an increased "probability of coalescence between particles of similar size" as well as an aggregation probability of smaller and larger particles that "decreases with increasing (particle) size caused by the increasing aggregation barrier". We can expect important, paradigm-shifting achievements in the coming years based on the concepts of sizedifferentiated growth as well as size-dependent aggregation and then even size-dependent agglomeration of those larger aggregates. These advances promise to displace perhaps completely the need for "instantaneous/burst nucleation" as a concept behind the formation of many if not most narrow particle-size dispersions. In short, even if the 70+ year era of dominance of the 1950 model is now due to close, the future for greater, mechanism-based, approaching molecular understanding of particle formation nucleation, growth, and agglomeration processes seems very bright indeed. It is hoped that the present review will help expedite the needed progress, research and associated new and exciting discoveries!

\section{Conflicts of interest}

The authors declare no competing financial interest.

\section{Acknowledgements}

Professor Samy El-Shall at Virginia Common Wealth University is thanked for sharing his expertise ${ }^{30,31}$ and helpful insights regarding classical nucleation theory. An anonymous, critical referee is thanked for their effort, insights, and valuable suggestions. A second, energetic, detailed referee is also thanked for their extensive, thoughtful and critical comments that helped improve the final manuscript. This work was supported at Colorado State University revised by the U.S. Department of Energy (DOE), Office of Science, Office of Basic Energy Sciences, Division of Chemical Sciences, Geosciences \& Biosciences, Catalysis Program, via DOE grant SE-FG402-02ER15453.

\section{References}

1 V. K. LaMer and R. H. Dinegar, Theory, Production and Mechanism of Formation of Monodispersed Hydrosols, J. Am. Chem. Soc., 1950, 72, 4847-4854. The number of citations given refers to a March 2019 Web of Science literature search.

2 C. B. Whitehead, S. Özkar and R. G. Finke, LaMer's 1950 Model for Particle Formation of Instantaneous Nucleation and Diffusion-Controlled Growth: A Historical Look at the Model's Origins, Assumptions, Equations, and Underlying Sulfur Sol Formation Kinetics Data, Chem. Mater., 2019, 31, 7116-7132.

3 M. A. Watzky and R. G. Finke, Transition Metal Nanocluster Formation Kinetic and Mechanistic Studies. A New Mechanism When Hydrogen Is the Reductant: Slow, Continuous Nucleation and Fast Autocatalytic Surface Growth, J. Am. Chem. Soc., 1997, 43, 10382-10400. Please also see references cited therein.

4 V. K. La Mer, Nucleation in Phase Transitions, Ind. Eng. Chem., 1952, 44, 1270-1277.

5 J. Turkevich, P. C. Stevenson and J. Hillier, A Study of the Nucleation and Growth Processes in the Synthesis of Colloidal Gold, Discuss. Faraday Soc., 1951, 11, 55-75.

6 J. Turkevich, P. C. Stevenson and J. Hillier, The Formation of Colloid Gold, J. Phys. Chem., 1953, 57, 670-673.

7 Y. Lin and R. G. Finke, Novel Polyoxoanion-and $\mathrm{Bu}_{4} \mathrm{~N}^{+}-$ Stabilized, Isolable, and Redissolvable, 20-30 ̊ Ir $\sim 300-900$ Nanoclusters: The Kinetically Controlled Synthesis, Characterization, and Mechanism of Formation of Organic Solvent-Soluble, Reproducible Size, and Reproducible Catalytic Activity Metal Nanoclusters, J. Am. Chem. Soc., 1994, 116, 8335-8353.

8 E. Matijevic, Preparation and Properties of Uniform Size Colloids, Chem. Mater., 1993, 5, 412-426.

9 D. Kashchiev, Nucleation: Basic Theory with Applications, Butterworth Heinemann, Oxford, 2000.

10 D. W. Oxtoby, Homogeneous Nucleation: Theory and Experiment, J. Phys.: Condens. Matter, 1992, 4, 7627-7650.

11 Nucleation-A Transition State to the Directed Assembly of Materials, Faraday Discuss., 2015, 179, 9-488.

12 R. Zhang, A. Khalizov, L. Wang, L. Hu and W. Xu, Nucleation and Growth of Nanoparticles in the Atmosphere, Chem. Rev., 2012, 112, 1957-2011. Please also see references cited therein.

13 S. Karthika, T. K. Radhakrishnan and P. Kalaichelvi, A Review of Classical and Nonclassical Nucleation Theories, Cryst. Growth Des., 2016, 16, 6663-6681.

14 E. E. Finney and R. G. Finke, Nanocluster Nucleation and Growth Kinetic and Mechanistic Studies: A Review Emphasizing Transition-Metal Nanoclusters, J. Colloid Interface Sci., 2008, 317-374.

15 N. T. K. Thanh, N. Maclean and S. Mahiddine, Mechanisms of Nucleation and Growth of Nanoparticles in Solution, Chem. Rev., 2014, 114, 7610-7630. This review is, in our opinion, superficial, often uncritical, and misses key literature. Five specific reasons supporting this opinion, and hence why 
this 2014 review should be viewed and used only with considerable caution in our opinion, are provided in the Supporting Information available with a prior paper ${ }^{27}$.

16 D. Erdemir, A. Y. Lee and A. S. Myerson, Nucleation of Crystals from Solution: Classical and Two-Step Models, Acc. Chem. Res., 2009, 42, 621-629.

17 W. W. Laxson and R. G. Finke, Nucleation is Second Order: An Apparent Kinetically Effective Nucleus of Two for $\operatorname{Ir}(0)_{n}$ Nanoparticle Formation from $\left[(1,5-\mathrm{COD}) \mathrm{Ir}^{\mathrm{I}} \cdot \mathrm{P}_{2} \mathrm{~W}_{15} \mathrm{Nb}_{3} \mathrm{O}_{62}\right]^{8-}$ Plus Hydrogen, J. Am. Chem. Soc., 2014, 136, 17601-17615. Please also see references cited therein.

18 B. Peters, Common Features of Extraordinary Rate Theories, J. Phys. Chem. B, 2015, 119, 6349-6356.

$19 \mathrm{~J}$. W. Gibbs, On the Equilibrium of Heterogeneous Substances, New Haven Trans. Conn. Acad. Arts Sci., 1874, III, 343-523.

20 L. Farkas, The velocity of nucleus formation in supersaturated vapors, $Z$. Phys. Chem., 1927, 125, 236-242.

21 M. Volmer and A. Z. Weber, Keimbildung in übersättigten Gebilden, Z. Phys. Chem., 1926, 119, 277-301.

22 M. Volmer, Kinetik der Phasenbildung, Verlag von Theodor Steinkopff, Leipzig, 1939.

23 R. Becker and W. Döring, Kinetische Behandlung der Keimbildung in übersättigten Dampfen, Ann. Phys., 1935, 24, 719-752.

24 J. Frenkel, Kinetic Theory of Liquids, Oxford University Press, New York, 1946, ch. VII.

25 J. B. Zeldovich, On the Theory of New Phase Formation: Cavitation, Acta Physicochim. URSS, 1943, 18, 1.

26 S. Özkar and R. G. Finke, Nanoparticle Nucleation is Termolecular and Involves Hydrogen: Evidence for a Kinetically Effective Nucleus of Three, $\left\{\mathrm{Ir}_{3} \mathrm{H}_{2 \mathrm{x}} \cdot \mathrm{P}_{2} \mathrm{~W}_{15} \mathrm{Nb}_{3} \mathrm{O}_{62}\right)^{6-}$, in $\operatorname{Ir}(0)_{n}$ Nanopaticle Formation From [(1,5-COD) $\operatorname{Ir}^{\mathrm{I}}$. $\left.\mathrm{P}_{2} \mathrm{~W}_{15} \mathrm{Nb}_{3} \mathrm{O}_{62}\right]^{8-}$ Plus Hydrogen, J. Am. Chem. Soc., 2017, 139, 5444-5457. See also references cited therein.

27 C. B. Whitehead and R. G. Finke, Nucleation Kinetics and Molecular Mechanism in Transition-Metal Nanoparticle Formation: The Intriguing, Informative Case of a Bimetallic Precursor, $\left\{\left[(1,5-\mathrm{COD}) \operatorname{Ir}^{\mathrm{I}} \cdot \mathrm{HPO}_{4}\right]_{2}\right\}^{2-}$, Chem. Mater., 2019, 31, 2848-2862.

28 H. Reiss, Comments on Clusters in Beams, Spectroscopy and Transitions, NATO ASI Series, 1986, 184, 115-133. See for example p. 118 regarding "the nucleus forms by a series of highly reversible additions of molecules", and the monomer-addition eqn (4) on p. 120.

29 D. Oxtoby, Nucleation of First-Order Transitions, Acc. Chem. Res., 1998, 31, 91-97.

30 M. Rusyniak, V. Abdelsayed, J. Campbell and M. S. El-Shall, Vapor Phase Homogeneous Nucleation of Higher Alkanes: Dodecane, Hexadecane, and Octadecane. 1. Critical Supersaturation and Nucleation Rate Measurements, J. Phys. Chem. B, 2001, 105, 11866-11872.

31 M. Rusyniak and M. S. El-Shall, Vapor Phase Homogeneous Nucleation of Higher Alkanes: Dodecane, Hexadecane, and Octadecane. 2. Corresponding States and Scaling Law Analysis, J. Phys. Chem. B, 2001, 105, 11873-11879.
32 Iridium(0) metal is known to be insoluble, or at best sparingly soluble. It is reported in the CRC handbook that it is only partially soluble in aqua regia, CRC Handbook of Chemistry and Physics, ed. W. M. Haynes, CRC Press, Boca Raton, FL, 95th edn, 2014.

33 H. Laksmono, S. Tanimura and B. E. Wyslouzil, Methanol nucleation in a supersonic nozzle, J. Chem. Phys., 2011, 135, 074305.

34 N. M. Dixit and C. F. Zukoski, Nucleation Rates and Induction Times During Colloidal Crystallization: Links Between Models and Experiments, Phys. Rev. E: Stat., Nonlinear, Soft Matter Phys., 2002, 66, 051602.

35 S. D. Lubetkin, Why Is It Much Easier to Nucleate Gas Bubbles than Theory Predicts?, Langmuir, 2003, 19, 2575-2587.

36 S. M. Kathmann, G. K. Schenter, B. C. Garrett, B. Chen and J. I. Siepmann, Thermodynamics and Kinetics of Nanoclusters Controlling Gas-to-Particle Nucleation, J. Phys. Chem. $C, 2009,113,10354-10370$. See also the ref. 16 and 25-37 therein concerning DFT studies that strive to go beyond classical nucleation theory.

37 S. M. Kathmann, G. K. Schenter and B. C. Garrett, Understanding the Sensitivity of Nucleation Kinetics, J. Chen. Phys., 2002, 116, 5046-5057.

38 A. S. Myerson, Concluding remarks, Faraday Discuss., 2015, 179, 543-547.

39 S. Karthika, T. K. Radhakrishnan and P. Kalaichelvi, A Review of Classical and Nonclassical Nucleation Theories, Cryst. Growth Des., 2016, 16, 6663-6681.

40 P. R. ten Wolde and D. Frenkel, Enhancement of Protein Crystal Nucleation by Critical Density Fluctuations, Science, 1997, 277, 1975-1978.

41 R. Zhang, A. Khalizov, L. Wang, M. Hu and W. Xu, Nucleation and Growth of Nanoparticles in the Atmosphere, Chem. Rev., 2012, 112, 1957-2011.

42 M. Avrami, Kinetics of Phase Change. I. General Theory, J. Chem. Phys., 1939, 7, 1103-1112.

43 M. Avrami, Kinetics of Phase Change. II. TransformationTime Relations for Random Distribution of Nuclei, J. Chem. Phys., 1940, 8, 212-224.

44 M. Avrami, Kinetics of Phase Change. III. Granulation, Phase Change, and Microstructure, J. Chem. Phys., 1941, 9, 177-184.

45 A. N. Kolmogorov, On the Statistical Theory of Metal Crystallization, Bull. Acad. Sci. USSR, Phys. Ser., 1937, 3, 355-360.

46 W. A. Johnson and R. F. Mehl, Reaction Kinetics in Processes of Nucleation and Growth, Trans. AIME, 1939, 135, 416-458.

47 B. V. Erofe'ev, A Generalized Equation of Chemical Kinetics and its Application in Reactions Involving Solids, Dokl. Akad. Sci. USSR, 1946, 52, 511-514.

48 E. E. Finney and R. G. Finke, Is There a Minimal Chemical Mechanism Underlying Classical Avrami-Erofe'ev Treatments of Phase Transformation Kinetic Data?, Chem. Mater., 2009, 21, 4692-4705. See also the ESI $\dagger$ accompanying this 2009 paper for a section and references on "A more detailed look at the A-E model" that contains 
an introduction to A-E, KJMA and related equations and their approximations and assumptions.

49 B. G. Hillis, B. P. Losey, J. Weng, N. Ghaleb, F. Hou and J. D. Martin, From Rate Measurements to Mechanistic Data for Condensed Matter Reactions: A Case Study Using the Crystallization of $\left[\mathrm{Zn}\left(\mathrm{OH}_{2}\right)_{6}\right]\left[\mathrm{ZnCl}_{4}\right]$, Crystals, 2017, 7, 11.

50 M. Fanfoni and M. Tomellini, The Johnson-Mehl-AvramiKolmogorov model: A brief review, Nuovo Cimento D, 1998, 20, 1171-1182.

51 R. G. Finke, M. A. Watzky and C. B. Whitehead, Response to: "Particle Size is a Primary Determinant for Sigmoidal Kinetics of Nanoparticle Formation: A "Disproof" of the Finke-Watzky (F-W) Nanoparticle Nucleation and Growth Mechanism”, Chem. Mater., 2020, 32, 3657-3672.

52 J. D. Martin, Comment: Particle Size is a Primary Determinant for Sigmoidal Kinetics of Nanoparticle Formation: A "Disproof" of the Finke-Watzky (F-W) Nanoparticle Nucleation and Growth Mechanism, Chem. Mater., 2020, 32, 3651-3656.

53 R. Szabó and G. Lente, A Comparison of the Stochastic and Deterministic Approaches in a Nucleation-Growth Type Model of Nanoparticle Formation, Chem. Mater., 2020, submitted.

54 T. G. Kurtz, The Relationship Between Stochastic and Deterministic Models for Chemical Reactions, J. Chem. Phys., 1972, 57, 2976-2978.

55 B. J. Hornstein and R. G. Finke, Transition-Metal Nanocluster Kinetic and Mechanistic Studies Emphasizing Nanocluster Agglomeration: Demonstration of a Kinetic Method That Allows Monitoring All Three Phases of Nanocluster Formation and Aging, Chem. Mater., 2004, 16, 139-150. (See also the addition/correction: Chem. Mater., 2004, 16, 3972).

56 C. Besson, E. E. Finney and R. G. Finke, A Mechanism for Transition-Metal Nanoparticle Self-Assembly, J. Am. Chem. Soc., 2005, 127, 8179-8184.

57 C. Besson, E. E. Finney and R. G. Finke, Nanocluster Nucleation, Growth and Then Agglomeration Kinetic and Mechanistic Studies: A More General, Four-Step Mechanism Involving Double Autocatalysis, Chem. Mater., 2005, 17, 4925-4938.

58 E. E. Finney and R. G. Finke, The Four-Step, DoubleAutocatalytic Mechanism for Transition-Metal Nanocluster Nucleation, Growth and Then Agglomeration: Metal, Ligand, Concentration, Temperature, and Solvent Dependency Studies, Chem. Mater., 2008, 20, 1956-1970.

59 T. O. Drews, M. A. Katsoulakis and M. Tsapatsis, Mathematical Model for Crystal Growth by Aggregation of Precursor Metastable Nanoparticles, J. Phys. Chem. B, 2005, 109, 23879-23887. Note that the authors preferred their more complex, 4-step Mechanism II (that has a typo in the second step; it should be $\mathrm{B} \rightarrow \mathrm{C}_{1}$, and not the repetitive $\mathrm{B}+$ $\left.\mathrm{C}_{\mathrm{i}} \rightarrow \mathrm{C}_{i+1}\right)$.

60 P. D. Kent, J. E. Mondloch and R. G. Finke, A Four-Step Mechanism for the Formation of Supported-Nanoparticle Heterogeneous Catalysts in Contact with Solution: The Conversion of $\operatorname{Ir}(1,5-\mathrm{COD}) \mathrm{Cl} / \gamma-\mathrm{Al}_{2} \mathrm{O}_{3}$ to $\operatorname{Ir}(0)_{\sim 170} / \gamma-\mathrm{Al}_{2} \mathrm{O}_{3}$, J. Am. Chem. Soc., 2014, 136, 1930-1941.
61 D. R. Handwerk, P. D. Shipman, C. B. Whitehead, S. Özkar and R. G. Finke, Mechanism-Enabled Population Balance Modeling of Particle Formation en Route to Particle Average Size and Size Distribution Understanding and Control, J. Am. Chem. Soc., 2019, 141, 15827-15839.

62 D. R. Handwerk, P. D. Shipman, C. B. Whitehead, S. Özkar and R. G. Finke, Particle Size Distributions via MechanismEnabled Population Balance Modeling, J. Phys. Chem. C, 2020, 14, 4852-4880.

63 S. Mozaffari, W. Li, C. Thompson, S. Ivanov, S. Seifert, B. Lee, L. Kovarik and A. M. Karim, Colloidal nanoparticle size control: experimental and kinetic modeling investigation of the ligand-metal binding role in controlling the nucleation and growth kinetics, Nanoscale, 2017, 9, 13772-13785.

64 A. M. Morris, M. A. Watzky and R. G. Finke, Protein aggregation kinetics, mechanism, and curve-fitting: a review of the literature, Biochim. Biophys. Acta, Proteins Proteomics, 1794, 2009, 375-397. Please also see the references therein.

65 K.-J. Kim, C.-H. Lee and J.-H. Shin, Characteristics of Cu(II) Precipitation by the Liquid Phase Reaction with Sulfide, Hwahak Konghak, 1991, 29, 639-645. A thorough search of the literature revealed little precedent for nucleation reaction orders greater than 1st or 2nd order. The Kim, at al. article listed is one of the highest claimed reaction orders we could find, a CuS system with a claimed 5th-order nucleation.

66 J. A. Christiansen and A. E. Neilsen, On the Kinetics of Formation of Precipitates of Sparingly Soluble Salts, Acta Chem. Scand., 1951, 5, 673-674.

67 V. K. La Mer and R. H. Dinegar, The Limiting Degrees of Supersaturation of the Sparingly Soluble Sulfates, J. Am. Chem. Soc., 1951, 73, 380-385.

68 In the 1950s, the state-of-the-art technique to collect particle size data was using turbidity/light scattering techniques that had a particle radius limit of detection of $100 \mathrm{~nm} .{ }^{1,2}$ Particles with a radius of $100 \mathrm{~nm}$ contain $\mathrm{ca}$. $10^{7-8}$ atoms! The limit of detection is approximately $10^{3}$ orders of magnitude better than what the naked eye is able to see. Meanwhile, modern microscopic and synchrotron techniques are able to observe particle formation at the $0.5-1.0 \mathrm{~nm}$ scale-an improvement of $10^{2}$ orders of magnitude in the ensuing 70 years, and an improvement necessary to directly observe the nucleation process that can involve as little as 2-3 primary element atoms. ${ }^{3,17,27}$.

69 The estimate was achieved as follows: the number of $\mathrm{BaSO}_{4}$ units in a nearly spherical particle of 100 micron size, is $n=\left(N_{0} \rho(4 / 3) \pi(D / 2) 3\right) / W$, where $n=$ number of $\mathrm{BaSO}_{4}$ units, $N_{0}=6.0^{22} \times 10^{23} \mathrm{~mol}^{-1}, \rho=$ room-temperature density of $\mathrm{BaSO}_{4}\left(4.49 \mathrm{~g} \mathrm{~cm} \mathrm{~cm}^{-3}\right.$; CRC Handbook of Chemistry and Physics, 77th edn; Lide, D. R., Frederikse, H. P. R., Eds.; CRC Press: Boca Raton, FL, 1996), $D=$ diameter of $\mathrm{BaSO}_{4}$ particles, and $W=$ formula weight of $\mathrm{BaSO}_{4}\left(233.38 \mathrm{~g} \mathrm{~mol}{ }^{-1}\right)$. From this one calculates 
$n=\left(6.022 \times 1023 \mathrm{~mol}^{-1}\right)\left(4.49 \mathrm{~g} \mathrm{~cm}^{-3}\right)(4 / 3) \pi\left((100 / 2) \times 10^{-4}\right.$ $\mathrm{cm})^{3} /\left(233.38 \mathrm{~g} \mathrm{~mol}^{-1}\right)$, that is, $n=6.0 \times 10^{15}$.

70 L. Bentea, M. A. Watzky and R. G. Finke, Sigmoidal Nucleation and Growth Curves Across Nature Fit by the Finke-Watzky Model of Slow Continuous Nucleation and Autocatalytic Growth: Explicit Formulas for the Lag and Growth Times Plus Other Key Insights, J. Phys. Chem. C, 2017, 121, 5302-5312.

71 D. Turnbull, The kinetics of precipitation of barium sulfate from aqueous solution, Acta Metall., 1953, 1, 684-691.

72 D. Gebauer, A. Völkel and H. Cölfen, Stable Prenucleation Calcium Carbonate Clusters, Science, 2008, 322, 1819-1822.

73 F. L. Eisele and D. R. Hanson, First Measurement of Prenucleation Molecular Clusters, J. Phys. Chem. A, 2000, 104, 830-836.

74 E. M. Pouget, P. H. H. Bomans, J. A. C. M. Goos, P. M. Frederik, G. de With and N. A. J. M. Sommerdijk, The Initial Stages of Template-Controlled $\mathrm{CaCO}_{3}$ Formation Revealed by Cryo-TEM, Science, 2009, 323, 1455-1458.

75 D. Gebauer, M. Kellermeir, J. D. Gale, L. Bergström and H. Cölfen, Pre-nucleation clusters as solute precursors in crystallization, Chem. Soc. Rev., 2014, 43, 2348-2371.

$76 \mathrm{~J}$. Reichenbach and K. Wynne, Frustration vs. Prenucleation: Understanding the Surprising Stability of Supersaturated Sodium Thiosulfate Solutions, J. Phys. Chem. C, 2018, 122, 7590-7596.

77 S. Fermani, C. Vettraino, I. Bonacini, M. Marcaccio, G. Falini, J. A. Gavira and J. M. Garcia Ruiz, Heterogeneous Crystallization of Proteins: Is it a Prenucleation Clusters Mediated Process?, Gryst. Gorwth Des., 2013, 13, 3110-3115.

78 F. Hou and J. D. Martin, Isotope Effects Reveal the Template Influence on the Crystal Growth of a Metal-Halide Network, J. Phys. Chem. C, 2019, 123, 7475-7485.

79 F. Hou, J. D. Martin, E. D. Dill, J. C. W. Folmer and A. A. Josey, Transition Zone Theory of Crystal Growth and Viscosity, Chem. Mater., 2015, 27, 3526-3532.

$80 \mathrm{~J}$. D. Martin and F. Hou, Transition zone theory of glass transition, J. Non-Cryst. Solids, 2018, 491, 24-33.

81 R. K. Ramamoorthy, E. Yildirim, E. Barba, P. Roblin, J. A. Vargas, L.-M. Lacroix, I. Rodriguez-Ruiz, P. Decorse, V. Petkiv, S. Teycheném and G. Viau, The Role of Pre-Nucleation Clusters in the Crystallization of Gold Nanoparticles, Nanoscale, 2020, 12, 16173-16188.

$82 \mathrm{~J}$. Halpern, Some aspects of chemical dynamics in solution, J. Chem. Ed., 1968, 45, 372-380.

83 G. Dulz and N. Sutin, The effect of chloride ions on the kinetics of the oxidation of chromium(II) by iron(III), J. Am. Chem. Soc., 1964, 86, 829-832.

$84 \mathrm{H}$. Freund, An Introduction to the Study of Enzymes, Wiley, New York, 1965, ch. 2. Note that presteady-state kinetics studies of enzymes are well known.

85 S. E. Smith, J. M. Sasaki, R. G. Bergman, J. E. Mondloch and R. G. Finke, Platinum-Catalyzed Phenyl and Methyl Group Transfer from Tin to Iridium: Evidence for an
Autocatalytic Reaction Pathway with an Unusual Preference for Methyl Transfer, J. Am. Chem. Soc., 2008, 130, 1839-1841.

86 J. E. Mondloch, Q. Wang, A. I. Frenkel and R. G. Finke, Development Plus Kinetic and Mechanistic Studies of a Prototype Supported-Nanoparticle Heterogeneous Catalyst Formation System in Contact with Solution: $\operatorname{Ir}(1,5-\mathrm{COD}) \mathrm{Cl} /$ $\gamma-\mathrm{Al}_{2} \mathrm{O}_{3}$ and Its Reduction by $\mathrm{H}_{2}$ to $\operatorname{Ir}(0)_{n} / \gamma-\mathrm{Al}_{2} \mathrm{O}_{3}, J$. Am. Chem. Soc., 2010, 132, 9701-9714.

87 A. M. Morris, M. A. Watzky, J. N. Agar and R. G. Finke, Fitting neurological protein aggregation kinetic data via a 2-step, minimal/"'Ockham's razor" model: the FinkeWatzky mechanism of nucleation followed by autocatalytic surface growth, Biochem., 2008, 47, 2413-2427.

88 N. A. Oladoja, A critical review of the applicability of Avrami fractional kinetic equation in adsorption-based water treatment studies, Desalin. Water Treat., 2015, 57, 15813-15825.

89 S. Özkar and R. G. Finke, Silver Nanoparticles Synthesized by Microwave Heating: A Kinetic and Mechanistic Re-Analysis and Re-Interpretation, J. Phys. Chem. C, 2017, 121, 27643-27654.

90 M. A. Watzky, E. E. Finney and R. G. Finke, TransitionMetal Nanocluster Size vs Formation Time and the Catalytically Effective Nucleus Number: A Mechanism-Based Treatment, J. Am. Chem. Soc., 2008, 130, 11959-11969.

91 S. R. K. Perala and S. Kumar, On the Two-Step Mechanism for Synthesis of Transition-metal Nanoparticles, Langmuir, 2014, 30, 12703-12711.

92 H. A. Brown, S. Xiong, G. A. Medvedev, Y. A. Chang, M. M. Abu-Omar, J. M. Caruthers and R. M. Waymouth, Zwitterionic Ring-Opening Polymerization: Models for Kinetics of Cyclic Poly(caprolactone) Synthesis, Macromolecules, 2014, 47, 2955-2963. Noteworthy in this paper are: (i) the similar approach of a disproof-based, minimalistic mechanism to start; (ii) the slow initiation, fast propagation polymerization system; (iii) the monomer addition steps in the mechanism; and (iv) the finding that such a system can lead to a relatively narrow size distribution-all features closely paralleling those in the present, nanoparticle ME-PBM study. Two major differences, however, are: (iv) the underlying mechanism is fundamentally different than any in the present nanoparticle study), and (v) a Monte Carlo, stochastic simulation had to be used due to the high polymer MWs and, hence, many monomer addition steps (vs the full ODE approach possible for our nanoparticle formation reaction and its order of magnitude fewer number of steps). Nevertheless, this excellent work is an example that fits our definition of ME-PBM, albeit now in polymer chemistry, something that expands the scope and impact of the concept of Mechanism-Enabled PBModeling. We thank Prof. Waymouth for stimulating discussion of the PBMs of polymer vs particle formation systems.

93 C. An, S. Wang, Y. Sun, Q. Zhang, J. Zhang, C. Wang and J. Fang, Plasmonic silver incorporated silver halides for efficient photocatalysis, J. Mater. Chem. A, 2016, 4, 4336-4352. 
94 G.-L. Wang, X.-F. Xu, L. Qiu, Y.-M. Dong, Z.-J. Li and C. Zhang, Dual Responsive Enzyme Mimicking Activity of AgX (X = Cl, Br, I) Nanoparticles and Its Application for Cancer Cell Detection, ACS Appl. Mater. Interfaces, 2014, 6, 6434-6442.

95 N. Durán, G. Nakazato and A. B. Seabra, Antimicrobial activity of biogenic silver nanoparticles, and silver chloride nanoparticles: an overview and comments, Appl. Microbiol. Biotechnol., 2016, 100, 6555-6570.

96 R. Ginell, A. M. Ginell and P. E. Spoerri, Association phenomena. I. The growth of particles of silver chloride and the higher-order Tyndall effect, J. Colloid Sci., 1947, 2, 521-525.

97 R. H. Ottewill and R. F. Woodbridge, The preparation of monodisperse silver bromide and silver iodide sols, J. Colloid Sci., 1961, 16, 581-594.

98 M. J. Insley and G. D. Parfitt, Precipitation of Silver Chloride from Homogeneous Solution. Part 1. - Particle Size and Shape Characteristics, Trans. Faraday Soc., 1968, 64, 1945-1954.

99 T. Sugimoto, Underlying mechanisms in size control of uniform nanoparticles, J. Colloid Interface Sci., 2007, 309, 106-118.

100 T. Sugimoto, The Theory of the Nucleation of Monodisperse Particles in Open Systems and Its Application to $\mathrm{AgBr}$ Systems, J. Colloid Interface Sci., 1992, 150, 208-225.

101 T. Sugimoto, F. Shiba, T. Sekiguchi and H. Itoh, Spontaneous nucleation of monodisperse silver halide particles from homogeneous gelatin solution I: silver chloride, Colloids Surf., A, 2000, 164, 183-203.

102 T. Sugimoto and F. Shiba, Spontaneous nucleation of monodisperse silver halide particles from homogeneous gelatin solution II: silver bromide, Colloids Surf., A, 2000, 164, 205-215.

103 D. B. Chu, J. S. Owen and B. Peters, Nucleation and Growth Kinetics from LaMer Burst Data, J. Phys. Chem. A, 2017, 121, 7511-7517.

104 S. Zhao, D. Xu, H. Ma, Z. Sun and J. Guan, Controllable preparation and formation mechanism of monodispersed silica particles with binary sizes, J. Colloid Interface Sci., 2012, 388, 40-46.

105 Z. Li and Y. Sun, Silver chlorobromide nanoparticles with highly pure phases: synthesis and characterization, J. Mater. Chem. A, 2013, 1, 6786-6793.

106 H. M. Trimble, Solubilities of Salts in Ethylene Glycol and in Its Mixtures with Water, Ind. Eng. Chem., 1931, 23, 165-167.

107 F. Shiba and Y. Okawa, Relationship between Supersaturation Ratio and Supply Rate of Solute in the Growth Process of Monodisperse Colloidal Particles and Application to AgBr Systems, J. Phys. Chem. B, 2005, 109, 21664-21668.

108 Z. Li, J. S. Okasinski, D. J. Gosztola, Y. Ren and Y. Sun, Silver chlorobromide nanocubes with significantly improved uniformity: synthesis and assembly into photonic crystals, J. Mater. Chem. C, 2015, 3, 58-65.

109 H. Sato, T. Hirai and I. Komasawa, Mechanism of Formation of Silver Halide Particles in Reverse Micellar Systems, J. Chem. Eng. Jpn., 1996, 29, 501-507.
110 K. Kimijima and T. Sugimoto, Growth Mechanism of AgCl Nanoparticles in a Reverse Micelle System, J. Phys. Chem. B, 2004, 108, 3735-3738.

111 G. Ma, Background-Free In vivo Time Domain Optical Molecular Imaging Using Colloidal Quantum Dots, ACS Appl. Mater. Interfaces, 2013, 5, 2835-2844.

112 Y. E. Panfil, M. Oded and U. Banin, Colloidal Quantum Nanostructures: Emerging Materials for Display Applications, Angew. Chem., Int. Ed., 2018, 57, 4274-4295.

113 K. Das and S. Baruah, Quantum dots for solar energy harvesting, Curr. Sci., 2018, 115, 659-668.

114 S. Kundu and A. Patra, Nanoscale Strategies for Light Harvesting, Chem. Rev., 2017, 117, 712-757.

115 L. Jing, S. V. Kershaw, Y. Li, X. Huang, Y. Li, A. L. Rogach and M. Gao, Aqueous Based Semiconductor Nanocrystals, Chem. Rev., 2016, 116, 10623-10730.

116 L. S. Ott and R. G. Finke, Nanocluster Formation and Stabilization Fundamental Studies: Investigating "SolventOnly" Stabilization En Route to Discovering Stabilization by the Traditionally Weakly Coordinating Anion $\mathrm{BF}_{4}{ }^{-}$Plus High Dielectric Constant Solvents, Inorg. Chem., 2006, 45, 8382-8393.

117 M. N. O'Brien, M. R. Jones and C. A. Mirkin, The nature and implications of uniformity in the hierarchical organization of nanomaterials, Proc. Natl. Acad. Sci. U. S. A., 2016, 113, 11717-11725.

118 Q. Dai, Y. Zhang, Y. Wang, B. Zou, W. W. Yu and M. Z. Hu, Ligand Effects on Synthesis and Post-Synthetic Stability of PbSe Nanocrystals, J. Phys. Chem. C, 2010, 114, 16160-16167.

119 P. Reiss, M. Carrière, C. Lincheneau, L. Vaure and S. Tamang, Synthesis of Semiconductor Nanocrystals, Focusing on Nontoxic and Earth-Abundant Materials, Chem. Rev., 2016, 116, 10731-10819.

120 J. D. Aiken III and R. G. Finke, A review of modern transition-metal nanoclusters: their synthesis, characterization, and applications in catalysis, J. Mol. Catal. A: Chem., 1999, 145, 1-44.

121 B. M. Cossairt, Shining Light on Indium Phosphide Quantum Dots: Understanding the Interplay among Precursor Conversion, Nucleation, and Growth, Chem. Mater., 2016, 28, 7181-7189.

122 M. R. Friedfeld, D. A. Johnson and B. M. Cossairt, Conversion of InP Clusters to Quantum Dots, Inorg. Chem., 2019, 58, 803-810.

123 Y. Li, C. Pu and X. Peng, Surface activation of colloidal indium phosphide nanocrystals, Nano Res., 2017, 10, 941-958.

124 N. Pradhan, D. Reifsnyder, R. Xie, J. Aldana and X. Peng, Surface Ligand Dynamics in Growth of Nanocrystals, J. Am. Chem. Soc., 2007, 129, 9500-9509.

125 B. M. McMurtry, K. Qian, J. T. Teglasi, A. K. Swarnakar, J. DeRoo and J. S. Owen, Continuous Nucleation and Size Dependent Growth Kinetics of Indium Phosphide Nanocrystals, Chem. Mater., 2020, 32, 4358-4368.

126 O. B. Achorn, D. Franke and M. G. Bawendi, Seedless Continuous Injection Synthesis of Indium Phosphide 
Quantum Dots as a Route to Large Size and Low Dispersity, Chem. Mater., 2020, 32, 6532-6539.

127 R. Lai, C. Pu and X. Peng, On-Surface Reactions in the Growth of High-Quality CdSe Nanocrystals in Nonpolar Solutions, J. Am. Chem. Soc., 2018, 140, 9174-9183.

128 H. Lu and R. L. Brutchey, Tunable Room-Temperature Synthesis of Coinage Metal Chalcogenide Nanocrystals from N-Heterocyclic Carbene Synthons, Chem. Mater., 2017, 29, 1396-1403.

129 B. Li, X. Zhang, L. Li, M. Li, J. Xu and Y. Hong, White luminescence from CdS nanocrystals under the blue light excitation, J. Solid State Chem., 2014, 214, 108-111.

130 M. P. Hendricks, B. M. Cossairt and J. S. Owen, The Importance of Nanocrystal Precursor Conversion Kinetics: Mechanism of the Reaction between Cadmium Carboxylate and Cadmium Bis(diphenyldithiophosphinate), ACS Nano, 2012, 6, 10054-10062.

131 H.-B. Kim and D.-J. Jang, Precursor-dependent shape variation of wurtzite CdSe crystals in a microwave-assisted polyol process, CrystEngComm, 2012, 14, 6946-6951.

132 B. Abécassis, C. Bouet, C. Garnero, D. Constantin, N. Lequeux, S. Ithurria, B. Dubertret, B. R. Pauw and D. Pontoni, Real-Time in Situ Probing of High-Temperature Quantum Dots Solution Synthesis, Nano Lett., 2015, 15, 2620-2626.

133 Using the same equation from ref. 69, we were able to approximate the number of CdSe to be $c a$. 10 by using a density of $5.81 \mathrm{~g} \mathrm{~cm}^{-3}$ and a molecular weight of $191.385 \mathrm{~g} \mathrm{~mol}^{-1}$.

134 S. Nakashima, K. Kikushima and K. Mukai, Infrared emitting property and spherical symmetry of colloidal $\mathrm{PbS}$ quantum dots, J. Cryst. Growth, 2013, 378, 537-541.

135 W.-T. Wu, H. Liu, C. Dong, W.-J. Zheng, L.-L. Han, L. Li, S.-Z. Qiao, J. Yang and X.-W. Du, Gain High-Quality Colloidal Quantum Dots Directly from Natural Minerals, Langmuir, 2015, 31, 2251-2255.

136 R. Xie, Z. Li and X. Peng, Nucleation Kinetics vs Chemical Kinetics in the Initial Formation of Semiconductor Nanocrystals, J. Am. Chem. Soc., 2009, 131, 15457-15466.

137 S. Libert, V. Gorshkov, D. V. Goia, E. Matijević and V. Privman, Model of Controlled Synthesis of Uniform Colloid Particles: Cadmium Sulfide, Langmuir, 2003, 19, 10679-10683.

138 R. Garcia Rodriguez, M. P. Hendricks, B. M. Cossairt, H. Liu and J. S. Owen, Conversion Reactions of Cadmium Chalcogenide Nanocrystal Precursors, Chem. Mater., 2013, 25, 1233-1249.

139 L. Qu, W. Yu and X. Peng, In Situ Observation of the Nucleation and Growth of CdSe Nanocrystals, Nano Lett., 2004, 4, 465-469.

140 J. S. Owen, E. M. Chan, H. Liu and A. P. Alivisatos, Precursor Conversion Kinetics and the Nucleation of Cadmium Selenide Nanocrystals, J. Am. Chem. Soc., 2010, 132, 18206-18213.

141 S. Tamang, C. Lincheneau, Y. Hermans, S. Jeong and P. Reiss, Chemistry of InP Nanocrystal Syntheses, Chem. Mater., 2016, 28, 2491-2506.
142 A. Eshuis and C. A. J. Koning, The mechanism of particle formation during homogeneous precipitation of zinc sulfide, Colloid Polym. Sci., 1994, 272, 1240-1244.

143 H. Liu, W. Hu, F. Ye, Y. Ding and J. Yang, Growth mechanism of $\mathrm{Ag}_{2} \mathrm{~S}$ nanocrystals in a nonpolar organic solvent, RSC Adv., 2013, 3, 616-622.

144 X. Zhao, T. Liu, Y. Cui, X. Hou, Z. Liu, X. Dai, J. Kong, W. Shi and T. J. S. Dennis, Antisolvent-assisted controllable growth of fullerene single crystal microwires for organic field effect transistors and photodetectors, Nanoscale, 2018, 10, 8170-8179.

145 S. G. Kwon and T. Hyeon, Formation Mechanisms of Uniform Nanocrystals via Hot-Injection and Heat-Up Methods, Small, 2011, 7, 2685-2702.

146 P. L. Saldanha, V. Lesnyak and L. Manna, Large scale syntheses of colloidal nanomaterials, Nano Today, 2017, 12, 46-63.

147 K.-J. Kim, R. P. Oleksak, E. B. Hostetler, D. A. Peterson, P. Chandran, D. M. Schut, B. K. Paul, G. S. Herman and C.-H. Chang, Continuous Microwave-Assisted Gas-Liquid Segmented Flow Reactor for Controlled Nucleation and Growth of Nanocrystals, Cryst. Growth Des., 2014, 14, 5349-5355.

148 H. Xia, Y. Xiahou, P. Zhang, W. Ding and D. Wang, Revitalizing the Frens Method To Synthesize Uniform, Quasi-Spherical Gold Nanoparticles with Deliberately Regulated Sizes from 2 to $330 \mathrm{~nm}$, Langmuir, 2016, 32, 5870-5880.

149 J. Turkevich, Colloidal gold. Part I, Gold Bull., 1985, 18, 86-91.

150 M. K. Chow and C. F. Zukoski, Gold Sol Formation Mechanisms: Role of Colloidal Stability, J. Colloid Interface Sci., 1994, 165, 97-109.

151 P. Georgiev, A. Bojinova, B. Kostova, D. Momekova, T. Bjornholm and K. Balashev, Implementing atomic force microscopy (AFM) for studying kinetics of gold nanoparticle's growth, Colloids Surf., A, 2013, 434, 154-163.

152 M. Wuithschick, A. Birnbaum, S. Witte, M. Sztucki, U. Vainio, N. Pinna, K. Rademann, F. Emmerling, R. Kraehnert and J. Polte, Turkevich in New Robes: Key Questions Answered for the Most Common Gold Nanoparticle Synthesis, ACS Nano, 2015, 9, 7052-7071.

153 M. Harada and S. Kizaki, Formation Mechanism of Gold Nanoparticles Synthesized by Photoreduction in Aqueous Ethanol Solution of Polymers Using In Situ Quick Scanning X-ray Absorption Fine Structure and SmallAngle X-ray Scattering, Cryst. Growth Des., 2016, 16, 1200-1212.

154 J. Zhang, H. Li, Q. Kuang and Z. Xie, Toward Rationally Designing Surface Structures of Micro- and Nanocrystallites: Role of Supersaturation, Acc. Chem. Res., 2018, 51, 2880-2887.

155 X. Yin, M. Shi, J. Wu, Y.-T. Pan, D. L. Gray, J. A. Bertke and H. Yang, Quantitative Analysis of Different Formation Modes of Platinum Nanocrystals Controlled by Ligand Chemistry, Nano Lett., 2017, 17, 6146-6150. 
156 V. N. Richards, N. P. Rath and W. E. Buhro, Pathway from a Molecular Precursor to Silver Nanoparticles: The Prominent Role of Aggregative Growth, Chem. Mater., 2010, 22, 3556-3567.

157 G. Dzido, P. Markowski, A. Malachowska-Jutsz, K. Prusik and A. B. Jarzebski, Rapid continuous microwave-assisted synthesis of silver nanoparticles to achieve very high productivity and full yield: from mechanistic study to optimal fabrication strategy, J. Nanopart. Res., 2015, 17, 1-15.

158 B. Bhattarai, Y. Zaker, A. Atnagulov, B. Yoon, U. Landman and T. P. Bigioni, Chemistry and Structure of Silver Molecular Nanoparticles, Acc. Chem. Res., 2018, 51, 3104-3113.

159 T.-H. Yang, H.-C. Peng, S. Zhou, C.-T. Lee, S. Bao, Y.-H. Lee, J.-M. Wu and Y. Xia, Toward a Quantitative Understanding of the Reduction Pathways of a Salt Precursor in the Synthesis of Metal Nanocrystals, Nano Lett., 2017, 17, 334-340.

160 L. Wu, H. Lian, J. J. Willis, E. D. Goodman, I. S. McKay, J. Qin, C. J. Tassone and M. Cargnello, Tuning Precursor Reactivity toward Nanometer-Size Control in Palladium Nanoparticles Studied by in Situ Small Angle X-ray Scattering, Chem. Mater., 2018, 30, 1127-1135.

161 J. Wu, H. Qian, L. Lu, J. Fan, Y. Guo and W. Fang, Influence of Reduction Kinetics on the Preparation of Well-Defined Cubic Palladium Nanocrystals, Inorg. Chem., 2018, 57, 8128-8136.

162 M. A. Meshgi, M. Kriechbaum, S. Biswas, J. D. Holmes and C. Marschner, Synthesis of indium nanoparticles at ambient temperature; simultaneous phase transfer and ripening, J. Nanopart. Res., 2016, 18, 363-372.

163 M. Faraday, The Bakerian Lecture: Experimental Relations of Gold (and Other Metals) to Light, Philos. Trans. R. Soc. London, 1857, 147, 145-181.

164 X. Ji, X. Song, J. Li, Y. Bai, W. Yang and X. Peng, Size Control of Gold Nanocrystals in Citrate Reduction: The Third Role of Citrate, J. Am. Chem. Soc., 2007, 129, 13939-13948.

165 J. Wang, H. F. M. Boelens, M. B. Thathagar and G. Rothenberg, In Situ Spectroscopic Analysis of Nanocluster Formation, ChemPhysChem, 2004, 5, 93-98.

166 B.-K. Pong, H. I. Elim, J.-X. Chong, W. Ji, B. L. Trout and J.-Y. Lee, New Insights on the Nanoparticle Growth Mechanism in the Citrate Reduction of Gold(III) Salt: Formation of the $\mathrm{Au}$ Nanowire Intermediate and Its Nonlinear Optical Properties, J. Phys. Chem. C, 2007, 111, 6281-6287.

167 Y. Shiraishi, H. Tanaka, H. Sakamoto, N. Hayashi, Y. Kofuji, S. Ichikawa and T. Hirai, Synthesis of Au Nanoparticles with Benzoic Acid as Reductant and Surface Stabilizer Promoted Solely by UV Light, Langmuir, 2017, 33, 13797-13804.

168 S. A. Lindley, J. K. Cooper, M. D. Rojas-Andrade, V. Fung, C. J. Leahy, S. Chen and J. Z. Zhang, Highly Tunable Hollow Gold Nanospheres: Gaining Size Control and Uniform Galvanic Exchange of Sacrificial Cobalt Boride Scaffolds, ACS Appl. Mater. Interfaces, 2018, 10, 12992-13001.
169 J. Polte, T. T. Ahner, F. Delissen, S. Sokolov, F. Emmerling, A. F. Thunemann and R. Kraehnert, Mechanism of Gold Nanoparticle Formation in the Classical Citrate Synthesis Method Derived from Coupled In Situ XANES and SAXS Evaluation, J. Am. Chem. Soc., 2010, 132, 1296-1301.

170 J. Polte, R. Erler, A. F. Thünemann, S. Sokolov, T. T. Ahner, K. Rademann, F. Emmerling and R. Kraehnert, Nucleation and Growth of Gold Nanoparticles Studied via in situ Small Angle X-ray Scattering at Millisecond Time Resolution, ACS Nano, 2010, 4, 1076-1082.

171 J. Polte, M. Herder, R. Erler, S. Rolf, A. Fischer, C. Würth, A. F. Thünemann, R. Kraehnert and F. Emmerling, Mechanistic insights into seeded growth processes of gold nanoparticles, Nanoscale, 2010, 2, 2463-2469.

172 T. Yao, Z. Sun, Y. Li, Z. Pan, H. Wei, Y. Xie, M. Nomura, Y. Niwa, W. Yan, Z. Wu, Y. Jiang, Q. Liu and S. Wei, Insights into Initial Kinetic Nucleation of Gold Nanocrystals, J. Am. Chem. Soc., 2010, 132, 7696-7701.

$173 \mathrm{~J}$. Keizer, A theory of spontaneous fluctuations in macroscopic systems, J. Chem. Phys., 1975, 63, 398-403.

174 I. N. Levine, Physical Chemistry, McGraw-Hill, New York City, NY, 6th edn, 2009, pp. 101-103.

175 Z. Zhou, G. J. Bedwell, R. Li, S. Palchoudhury, P. E. Prevelige and A. Gupta, Pathways for Gold Nucleation and Growth over Protein Cages, Langmuir, 2017, 33, 5925-5931.

176 D. Zahn, Thermodynamics and Kinetics of Prenucleation Clusters, Classical and Non-Classical Nucleation, ChemPhysChem, 2015, 16, 2069-2075.

177 S. J. Khan, C. M. Sorensen and A. Chakrabarti, Computer Simulations of Nucleation of Nanoparticle Superclusters from Solution, Langmuir, 2012, 28, 5570-5579.

178 M. Kellermeier, R. Rosenberg, A. Moise, U. Anders, M. Przybylski and H. Cölfen, Amino acids form prenucleation clusters: ESI-MS as a fast detection method in comparison to analytical ultracentrifugation, Faraday Discuss., 2012, 159, 23-45.

179 T. Li, A. J. Senesi and B. Lee, Small Angle X-ray Scattering for Nanoparticle Research, Chem. Rev., 2016, 116, 11128-11180.

180 B. Ingham, X-ray Scattering Characterisation of Nanoparticles, Crystallogr. Rev., 2015, 21, 229-303.

181 X. Chen, J. Schröder, S. Hauschild, S. Rosenfeldt, M. Dulle and S. Förster, Simultaneous SAXS/WAXS/UV-Vis Study of the Nucleation and Growth of Nanoparticles: A Test of Classical Nucleation Theory, Langmuir, 2015, 31, 11678-11691.

182 A. I. Frenkel, Applications of extended X-ray absorption fine-structure spectroscopy to studies of bimetallic nanoparticle catalysts, Chem. Soc. Rev., 2012, 41, 8163-8178.

183 D. Koziej, Revealing Complexity of Nanoparticle Synthesis in Solution by in Situ Hard X-ray Spectroscopy-Today and Beyond, Chem. Mater., 2016, 28, 2478-2490.

184 K.-H. Yih, I. K. Hamdemir, J. M. Mondloch, E. Bayram, S. Özkar, R. Vasic, A. I. Frenkel, O. Anderson and R. G. Finke, Synthesis and Characterization of [(1,5-Cyclooctadiene) $\operatorname{Ir}(\mu-\mathrm{H})]_{4}$ : A Multipurpose, Tetrametallic, Coordinatively Unsaturated $\mathrm{Ir}_{4}$-Based Precatalyst and Synthon, Inorg. Chem., 2012, 51, 3186-3193. 
185 W. W. Laxson, S. Özkar, S. Folkman and R. G. Finke, The Story of a Mechanism-Based Solution to an Irreproducible Synthesis Resulting in an Unexpected Closed-System Requirement for the $\mathrm{LiBEt}_{3} \mathrm{H}$-Based Reduction: the Case of the Novel Subnanometer Cluster, $[\operatorname{Ir}(1,5-\mathrm{COD})(\mu-\mathrm{H})]_{4}$, and the Resulting Improved, Independently Repeatable, Reliable Synthesis, Inorg. Chim. Acta, 2015, 432, 250-257.

186 J. Polte, Fundamental growth principles of colloidal metal nanoparticles - a new perspective, CrystEngComm, 2015, 17, 6809-6830.

187 L. Starkey-Ott and R. G. Finke, Transition-Metal Nanocluster Stabilization for Catalysis: A Critical Review of Ranking Methods and Putative Stabilizers, Coord. Chem. Rev., 2007, 251, 1075-1100.

188 E. E. Finney and R. G. Finke, Catalyst Sintering Kinetic Data: Is There a Minimal Chemical Mechanism Underlying Kinetics Previously Fit by Empirical Power-Law Expressions-and if So, What Are Its Implications?, Ind. Eng. Chem. Res., 2017, 56, 10271-10286.

189 M. Harada and Y. Kamigaito, Nucleation and Aggregative Growth Process of Platinum Nanoparticles Studied by in Situ Quick XAFS Spectroscopy, Langmuir, 2012, 28, 2415-2428.

190 S. Yan, Z. Wu, H. Yu, Y. Gong, Y. Tan, R. Du, W. Chen, X. Xing, G. Mo, Z. Chen, Q. Cai and D. Sun, Time-Resolved Small-Angle X-ray Scattering Study on the Growth Behavior of Silver Nanoparticles, J. Phys. Chem. C, 2014, 118, 11454-11463.

191 A. Desireddy, B. E. Conn, J. Guo, B. Yoon, R. N. Barnett, B. M. Monahan, K. Kirschbaum, W. P. Griffith, R. L. Whetten, U. Landman and T. P. Bigioni, Ultrastable Silver Nanoparticles, Nature, 2013, 501, 399-402.

192 A. M. Karim, N. Al Hasan, S. Ivanov, S. Siefert, R. T. Kelly, N. G. Hallfors, A. Benavidez, L. Kovarik, A. Jenkins, R. E. Winans and A. K. Datye, Synthesis of $1 \mathrm{~nm} \mathrm{Pd}$ Nanoparticles in a Microfluidic Reactor: Insights from in Situ X-ray Absorption Fine Structure Spectroscopy and Small-Angle X-ray Scattering, J. Phys. Chem. C, 2015, 119, 13257-13267.

193 M. Harada, N. Tamura and M. Takenaka, Nucleation and Growth of Metal Nanoparticles during Photoreduction Using In Situ Time-Resolved SAXS Analysis, J. Phys. Chem. C, 2011, 115, 14081-14092.

194 P. Abellan, L. R. Parent, N. Al Hasan, C. Park, I. Arslan, A. M. Karim, J. E. Evans and N. D. Browning, Gaining Control over Radiolytic Synthesis of Uniform Sub-3-nanometer Palladium Nanoparticles: Use of Aromatic Liquids in the Electron Microscope, Langmuir, 2016, 32, 1468-1477.

195 N. Ortiz and S. E. Skrabalak, Manipulating Local Ligand Environments for the Controlled Nucleation of Metal Nanoparticles and their Assembly into Nanodendrites, Angew. Chem., Int. Ed., 2012, 51, 11757-11761.

196 M. Harada and Y. Inada, In Situ Time-Resolved XAFS Studies of Metal Particle Formation by Photoreduction in Polymer Solutions, Langmuir, 2009, 25, 6049-6061.

197 M. Saeki, D. Matsumura, T. Yomogida, T. Taguchi, T. Tsuji, H. Saitoh and H. Ohba, In Situ Time-Resolved XAFS
Studies on Laser-Induced Particle Formation of Palladium Metal in an Aqueous/EtOH Solution, J. Phys. Chem. C, 2019, 123, 817-824.

198 S. Özkar and R. G. Finke, Nanoparticle Formation Kinetics and Mechanistic Studies Important to Mechanism-Based Particle-Size Control: Evidence for Ligand-Based Slowing of the Autocatalytic Surface Growth Step Plus Postulated Mechanisms, J. Phys. Chem. C, 2019, 123, 14047-14057.

199 W. Stöber, A. Fink and E. Bohn, Controlled Growth of Monodisperse Silica Spheres in the Micron Size Range, J. Colloid Interface Sci., 1968, 26, 62-69.

200 K. D. Hartlen, A. P. T. Athanasopoulos and V. Kitaev, Facile Preparation of Highly Monodisperse Small Silica Spheres (15 to $>200 \mathrm{~nm}$ ) Suitable for Colloidal Templating and Formation of Ordered Arrays, Langmuir, 2008, 24, 1714-1720.

201 T. Matsoukas and E. Gulari, Self-sharpening Distributions Revisited Polydispersity in Growth by Monomer Addition, J. Colloid Interface Sci., 1991, 145, 557-562.

202 G. H. Bogush and C. F. Zukoski, Uniform Silica Particle Precipitation: An Aggregative Growth Model, J. Colloid Interface Sci., 1991, 142, 19-34.

203 Y. Han, Z. Lu, Z. Teng, J. Liang, Z. Guo, D. Wang, M.-Y. Han and W. Yang, Unraveling the Growth Mechanism of Silica Particles in the Stöber Method: In Situ Seeded Growth Model, Langmuir, 2017, 33, 5879-5890.

204 A. van Blaaderen, J. van Geest and A. Vrij, Monodisperse Colloidal Silica Spheres from Tetraalkoxysilanes: Particle Formation and Growth Mechanism, J. Colloid Interface Sci., 1992, 154, 481-501.

205 H. Giesche, Synthesis of Monodispersed Silica Powders I. Particle Properties and Reaction Kinetics, J. Eur. Ceram. Soc., 1994, 14, 189-204.

206 J.-L. Look, G. H. Bogush and C. F. Zukoski, Colloidal Interactions during the Precipitation of Uniform Submicrometre Particles, Faraday Discuss. Chem. Soc., 1990, 90, 345-357.

207 C. F. Zukoski, M. K. Chow, G. H. Bogush and J.-L. Look, Precipitation of Uniform Particles: The Role of Aggregation, Mater. Res. Soc. Symp. Proc., 1990, 180, 131-140.

208 C. F. Zukoski, J.-L. Look and G. H. Bogush, Formation of Uniform Precipitates from Alkoxides, Adv. Chem. Ser., 1994, 234, 451-465.

209 P. Calvert, And they all look just the same, Nature, 1994, 367, 119-120.

210 G. H. Bogush and C. F. Zukoski, Studies of the Kinetics of the Precipitation of Uniform Silica Particles through the Hydrolysis and Condensation of Silicon Alkoxides, J. Colloid Interface Sci., 1991, 142, 1-18.

211 J. K. Bailey and M. L. Mecartney, Mechanism of Silica and Titania Colloidal Particle Formation from Metal Alkoxide, Mater. Res. Soc. Symp. Proc., 1990, 180, 153-158.

212 J. K. Bailey and M. L. Mecartney, Formation of colloidal silica particles from alkoxides, Colloid Surf., 1992, 63, 151-161.

213 L. S. Ott and R. G. Finke, Transition-Metal Nanocluster Stabilization vs Agglomeration Fundamental Studies: 
A Quantitative Measurement of the Two Rate Constants for Agglomeration and Their Informative Temperature Dependence, Chem. Mater., 2008, 20, 2592-2601.

214 S. Shields, W. E. Buhro, E. E. Finney and R. G. Finke, Gold Nanocluster Agglomeration Kinetic Studies: Evidence for Parallel Bimolecular Plus Autocatalytic Agglomeration Pathways as a Mechanistic Alternative to an AvramiBased Analysis, Chem. Mater., 2012, 24, 1718-1725.

215 E. Bayram, J. Lu, C. Aydin, N. D. Browning, S. Özkar, E. E. Finney, B. C. Gates and R. G. Finke, Agglomerative Sintering of an Atomically Dispersed $\mathrm{Ir}_{1} /$ Zeolite Y Catalyst: Compelling Evidence Against Ostwald Ripening But for Bimolecular and Autocatalytic Agglomeration Catalyst Sintering Steps, ACS Catal., 2015, 5, 3514-3527.

216 S. Özkar and R. G. Finke, Palladium(0) Nanoparticle Formation, Stabilization, and Mechanistic Studies: $\mathrm{Pd}(\mathrm{acac})_{2}$ as a Preferred Precursor, $\left[\mathrm{Bu}_{4} \mathrm{~N}\right]_{2} \mathrm{HPO}_{4}$ Stabilizer, Plus the Stoichiometry, Kinetics, and Minimal, 4-Step Mechanism of the Palladium Nanoparticle Formation and Subsequent Agglomeration Reactions, Langmuir, 2016, 32, 3699-3716.

217 S. Özkar and R. G. Finke, A Classic, Azo-Dye Agglomeration System: Evidence for Slow, Continuous Nucleation, Autocatalytic Agglomerative Growth, Plus the Effects of Dust-Removal by Microfiltration on the Kinetics, J. Phys. Chem. A, 2017, 121, 7071-7078.

218 T. Yokoi, J. Wakabayashi, Y. Otsuka, W. Fan, M. Iwama, R. Watanabe, K. Aramaki, A. Shimojima, T. Tatsumi and T. Okubo, Mechanism of Formation of Uniform-Sized Silica Nanospheres Catalyzed by Basic Amino Acids, Chem. Mater., 2009, 21, 3719-3729.

219 C. C. M. C. Carcouët, M. W. P. van de Put, B. Mezari, P. C. M. M. Magusin, J. Laven, P. H. H. Bomans, H. Friedrich, A. C. C. Esteves, N. A. J. M. Sommerdijk, R. A. T. M. van Benthem and G. de With, Nucleation and Growth of Monodisperse Silica Nanoparticles, Nano Lett., 2014, 14, 1433-1438.

220 E. C. Vreeland, J. Watt, G. B. Schober, B. G. Hance, M. J. Austin, A. D. Price, B. D. Fellows, T. C. Monson, N. S. Hudak, L. Maldonado-Camargo, A. C. Bohorquez, C. Rinaldi and D. L. Huber, Enhanced Nanoparticle Size Control by Extending LaMer's Mechanism, Chem. Mater., 2015, 27, 6059-6066.

221 E. Matijević and P. Scheiner, Ferric Hydrous Oxide Sols: III. Preparation of Uniform Particles by Hydrolysis of Fe(III)Chloride, -Nitrate, and -Perchlorate Solutions, J. Colloid Interface Sci., 1978, 63, 509-524.

222 CRC Handbook of Chemistry and Physics, ed. D. R. Lide, CRC Press, Boca Raton, FL, 89th edn, 2008.

223 T. C. Chamberlin, Studies for Students. The Method of Multiple Working Hypotheses, J. Geol., 1897, 5, 837-848.

224 J. R. Platt, Strong Inference, Science, 1964, 146, 347-353. Platt and Chamberlin's method is of course a general method of thinking that has broad application far beyond just scientific endeavors.

225 S. L. Scott, The Burden of Disproof, ACS Catal., 2019, 9, 4706-4708.
226 T. Wen, X. Zhang, D. Zhang, C. Zhang, Q. Wen, H. Zhang and Z. Zhong, Simulation of Batch Nanoparticle Growth by the Generalized Diffusional Model, J. Phys. Chem. C, 2018, 122, 27070-27081.

227 S. Famiani, A. P. LaGrow, M. O. Besenhard, S. Maenosono and N. T. K. Thanh, Synthesis of Fine-Tuning Highly Magnetic $\mathrm{Fe} @ \mathrm{Fe}_{\mathrm{x}} \mathrm{O}_{\mathrm{y}}$ Nanoparticles through Continuous Injection and a Study of Magnetic Hyperthermia, Chem. Mater., 2018, 30, 8897-8904.

228 I. R. Epstein, The consequences of imperfect mixing in autocatalytic chemical and biological systems, Nature, 1995, 374, 321-327.

229 W. Baaziz, B. P. Pichon, S. Fleutot, Y. Liu, C. Lefevre, J.-M. Greneche, M. Toumi, T. Mhiri and S. Begin-Colin, Magnetic Iron Oxide Nanoparticles: Reproducible Tuning of the Size and Nanosized-Dependent Composition, Defects, and Spin Canting, J. Phys. Chem. C, 2014, 118, 3795-3810.

230 C. M. McGilvery, A. E. Goode, M. S. P. Shaffer and D. W. McComb, Contamination of holey/lacey carbon films in STEM, Micron, 2012, 43, 450-455.

231 S. Horiuchi, T. Hanada, M. Ebisawa, Y. Matsuda, M. Kobayashi and A. Takahara, Contamination-Free Transmission Electron Microscopy for High-Resolution Carbon Elemental Mapping of Polymers, ACS Nano, 2009, 3, 1297-1304.

232 S. G. Kwon and T. Hyeon, Colloidal Chemical Synthesis and Formation Kinetics of Uniformly Sized Nanocrystals of Metals, Oxides, and Chalcogenides, Acc. Chem. Res., 2008, 41, 1696-1709.

233 T. He, D. R. Chen, X. L. Jiao, Y. Wang and Y. Duan, Solubility-Controlled Synthesis of High-Quality $\mathrm{Co}_{3} \mathrm{O}_{4}$ Nanocrystals, Chem. Mater., 2005, 17, 4023-4030.

234 S. Yang and Q. Liu, Formation mechanism of apextruncated octahedral $\mathrm{Cu}_{2} \mathrm{O}$ microcrystal, CrystEngComm, 2016, 18, 8229-8236.

235 S. Arshadi, J. Moghaddam and M. Eskandarian, LaMer diagram approach to study the nucleation and growth of $\mathrm{Cu}_{2} \mathrm{O}$ nanoparticles using supersaturation theory. Korean, J. Chem. Eng., 2014, 31, 2020-2026.

236 P. Stolzenburg and G. Garnweitner, Experimental and numerical insights into the formation of zirconia nanoparticles: a population balance model for the nonaqueous synthesis, React. Chem. Eng., 2017, 2, 337-348.

237 J. K. Beattie, Monodisperse colloids of transition metal and lanthanide compounds, Pure Appl. Chem., 1989, 61, 937-941.

238 T. Okubo, K. Kobayashi, A. Kuno and A. Tsuchida, Kinetic study of the formation reaction of colloidal silica spheres by transmitted-light-intensity and dynamic light-scattering measurements, Colloid Polym. Sci., 1999, 277, 483-487.

239 J. Ng, S. Xu, X. Zhang, H. Y. Yang and D. D. Sun, Hybridized Nanowires and Cubes: A Novel Architecture of a Heterojunctioned $\mathrm{TiO}_{2} / \mathrm{SrTiO}_{3}$ Thin Film for Efficient Water Splitting, Adv. Funct. Mater., 2010, 20, 4287-4294.

240 H. Mehranpour, M. Askari, M. Sasani Ghamsari and H. Farzalibeik, Study on the Phase Transformation Kinetics of Sol-Gel Drived $\mathrm{TiO}_{2}$ Nanoparticles, J. Nanomater., 2010, 2010, 626978. 
241 Y. Huang and J. E. Pemberton, Synthesis of uniform, spherical sub-100 nm silica particles using a conceptual modification of the classic LaMer model, Colloids Surf., A, 2010, 360, 175-183.

242 C. Wang, H. Shpaisman, A. D. Hollingsworth and D. G. Grier, Celebrating Soft Matter's 10th Anniversary: Monitoring colloidal growth with holographic microscopy, Soft Matter, 2015, 11, 1062-1066.

243 L. Luo, Y. Liang, E. S. Erichsen and R. Anwander, Monodisperse mesoporous silica nanoparticles of distinct topology, J. Colloid Interface Sci., 2017, 495, 84-93.

244 J. K. Bailey, C. J. Brinker and M. L. Mecartney, Growth Mechanisms of Iron Oxide Particles of Differing Morphologies from the Forced Hydrolysis of Ferric Chloride Solutions, J. Colloid Interface Sci., 1993, 157, 1-13.

245 S. G. Kwon, Y. Piao, J. Park, S. Angappane, Y. Jo, N.-M. Hwang, J.-G. Park and T. Hyeon, Kinetics of Monodisperse Iron Oxide Nanocrystal Formation by "Heating-Up" Process, J. Am. Chem. Soc., 2007, 129, 12571-12584.

246 M. Drofenik, M. Kristl, A. Žnidaršič, D. Hanžel and D. Lisjak, Hydrothermal Synthesis of Ba-Hexaferrite Nanoparticles, J. Am. Ceram. Soc., 2007, 90, 2057-2061.

247 A. Lassenberger, T. A. Grunewald, P. D. J. van Oostrum, R. Rennhofer, H. Amenitsch, R. Zirbs, H. C. Lichteneggar and E. Reimhult, Monodisperse Iron Oxide Nanoparticles by Thermal Decomposition: Elucidating Particle Formation by Second-Resolved in Situ Small-Angle X-ray Scattering, Chem. Mater., 2017, 29, 4511-4522.

248 H. S. Dehsari, R. A. Harris, A. H. Ribeiro, W. Tremel and A. Asadi, Optimizing the Binding Energy of the Surfactant to Iron Oxide Yields Truly Monodisperse Nanoparticles, Langmuir, 2018, 34, 6582-6590.

249 S. Belaïd, D. Stanicki, L. Van der Elst, R. N. Muller and S. Laurent, Influence of experimental parameters on iron oxide nanoparticle properties synthesized by thermal decomposition: size and nuclear magnetic resonance studies, Nanotechnology, 2018, 29(165603), 1-12.

250 A. W. Jansons and J. E. Hutchison, Continuous Growth of Metal Oxide Nanocrystals: Enhanced Control of Nanocrystal Size and Radial Dopant Distribution, ACS Nano, 2016, 10, 6942-6951.

251 T. O. Drews, M. A. Katsoulakis and M. Tsapatsis, A Mathematical Model for Crystal Growth by Aggregation of Precursor Metastable Nanoparticles, J. Phys. Chem. B, 2005, 109, 23879-23887.

252 I. M. Lifshitz and V. V. Slyozov, The kinetics of precipitation from supersaturated solid solutions, J. Phys. Chem. Solids, 1961, 19, 35-50.

253 R. L. Penn and J. F. Banfield, Imperfect Oriented Attachment: Dislocation Generation in Defect-Free Nanocrystals, Science, 1998, 281, 969-971.

254 R. L. Penn and J. F. Banfield, Morphology development and crystal growth in nanocrystalline aggregates under hydrothermal conditions: Insights from titania, Geochim. Cosmochim. Acta, 1999, 63, 1549-1557.

255 R. L. Penn, Kinetics of Oriented Aggregation, J. Phys. Chem. $B, 2004,108,12707-12712$.
256 S. Auer and D. Frenkel, Prediction of absolute crystalnucleation rate in hard-sphere colloids, Nature, 2001, 409, 1020-1023.

257 D. Pan, Q. Wang and L. An, Controlled synthesis of monodisperse nanocrystals by a two-phase approach without the separation of nucleation and growth processes, J. Mater. Chem., 2009, 19, 1063-1073.

258 V. Privman, D. V. Goia, J. Park and E. Matijević, Mechanism of Formation of Monodispersed Colloids by Aggregation of Nanosize Precursors, J. Colloid Interface Sci., 1999, 213, 36-45.

259 T. Sugimoto, Formation of Monodispersed Nano- and Micro-Particles Controlled in Size, Shape, and Internal Structure, Chem. Eng. Technol., 2003, 26, 313-321.

260 D. L. Van Hyning, W. G. Klemperer and C. F. Zukoski, Silver Nanoparticle Formation: Predictions and Verification of the Aggregative Growth Model, Langmuir, 2001, 17, 3128-3135.

261 E. Matijević and D. Goia, Formation Mechanisms of Uniform Colloid Particles, Croat. Chem. Acta, 2007, 80, 485-491.

262 T. O. Drews and M. Tsapatsis, Model of the evolution of nanoparticles to crystals via an aggregative growth mechanism, Microporous Mesoporous Mater., 2007, 101, 97-107.

263 S. Kumar, T. M. Davis, H. Ramanan, R. L. Penn and M. Tsapatsis, Aggregative Growth of Silicalite-1, J. Phys. Chem. B, 2007, 111, 3398-3403.

264 F. Wang, V. N. Richards, S. P. Shields and W. E. Buhro, Kinetics and Mechanisms of Aggregative Nanocrystal Growth, Chem. Mater., 2014, 26, 5-21.

265 V. N. Richards, S. P. Shields and W. E. Buhro, Nucleation Control in the Aggregative Growth of Bismuth Nanocrystals, Chem. Mater., 2011, 23, 137-144.

266 D. T. Robb and V. Privman, Model of Nanocrystal Formation in Solution by Burst Nucleation and Diffusional Growth, Langmuir, 2008, 24, 26-35.

267 V. Privman, Mechanisms of Diffusional Nucleation of Nanocrystals and Their Self-Assembly into Uniform Colloids, Ann. N. Y. Acad. Sci., 2009, 1161, 508-525.

268 P. J. Skrdla, Use of Dispersive Kinetic Models for Nucleation and Denucleation to Predict Steady-State Nanoparticle Size Distributions and the Role of Ostwald Ripening, J. Phys. Chem. C, 2012, 116, 214-225.

269 S. St. John, Z. Nan, N. Hu, D. W. Schaefer and A. P. Angelopoulos, A nanoscale-modified LaMer model for particle synthesis from inorganic tin-platinum complexes, J. Mater. Chem. A, 2013, 1, 8903-8916.

270 S. R. K. Perala and S. Kumar, On the Mechanism of Metal Nanoparticle Synthesis in the Brust-Schiffrin Method, Langmuir, 2013, 29, 9863-9873.

271 F. Tong, M. P. Hanson and C. J. Bardeen, Analysis of reaction kinetics in the photomechanical molecular crystal 9-methylanthracene using an extended Finke-Watzky model, Phys. Chem. Chem. Phys., 2016, 18, 31936-31945.

272 A. B. Levit and R. L. Rowell, Time Dependence of the Size Distribution, Number Concentration and Surface Area in La Mer Sulfur Sols, J. Colloid Interface Sci., 1975, 50, 162-169. 
273 C. F. Zukoski, J.-L. Look and G. H. Bogush, Formation of Uniform Precipitates from Alkoxides. In The Colloid Chemistry of Silica, in Advances in Chemistry, ed. H. E. Bergna, American Chemical Society, Washington, DC, 1994, pp. 451-465.

274 A. P. LaGrow, B. Ingham, M. F. Toney and R. D. Tilley, Effect of Surfactant Concentration and Aggregation on the Growth Kinetics of Nickel Nanoparticles, J. Phys. Chem. C, 2013, 117, 16709-16718.

275 A. P. Sergievskaya, V. V. Tatarchuk, E. V. Makotchenko and I. V. Mironov, Formation of gold nanoparticles during the reduction of $\mathrm{HAuBr}_{4}$ in reverse micelles of oxyethylated surfactant: Influence of gold precursor on the growth kinetics and properties of the particles, J. Mater. Res., 2015, 30, 1925-1933.

276 The first thing apparent in analyzing the literature in the tables in the ESI $\dagger$ is (i) a failure to use consistently a proper scientific method of the disproof ${ }^{224,225}$ of multiple alternative hypotheses ${ }^{223}$ - with too many papers that don't disprove anything, but instead wind up with their (often explicitly unstated) hypothesis as their conclusion. This is not science; it is confirmation bias that in turn inhibits unbiased thinking and reliable science. Second, (ii) there is a pattern apparent in the tables in the ESI $\dagger$ of accepting and supporting theories/models/mechanisms based on their popularity. As stated in an excellent, recent ACS Catalysis editorial entitled "The Burden of Disproof", 225 "the validity of a mechanistic (or any scientific) hypothesis is not established by the strength of a consensus (i.e., what fraction of researchers believe it to be true at any given time)." Awareness of even just Turkevich's groundbreaking 1951 paper $^{5}$ gives one serious pause about blindly accepting and applying the burst-nucleation and diffusion-controlled growth 1950 model in the absence of strongly supportive evidence. It is upon us as modern researchers to decide if any scientific model is appropriate to our system or is simply being "inappropriately cited", a broader problem of "The classic theories work(ing) poorly or not at all when applied beyond the validity of their assumptions" as B. Peters has insightfully noted. ${ }^{18}$ Third, as mentioned in the main text (iii) a better consideration and understanding of the different types of models in science, ${ }^{277,278,279}$ including the strengths and weaknesses of those different types of models, would have helped expedite the understanding of particle formation in our opinion.

277 A. Gelfert, How to Do Science with Models: A Philosophical Primer, Springer, 2016.

278 E. E. Finney and R. G. Finke, Fitting and Interpreting Transition-Metal Nanocluster Formation and Other Sigmoidal Kinetic Data: A More Thorough Testing of Dispersive Kinetics vs Chemical-Mechanism-Based Equations and Treatments for 4-Step Type Kinetic Data, Chem. Mater., 2009, 21, 4468-4479. See the discussion and references in the ESI $\dagger$ on different types of models in science.
279 F. Hou, J. D. Martin, E. D. Dill, J. C. W. Folmer and A. A. Josey, Transition Zone Theory of Crystal Growth and Viscosity, Chem. Mater., 2015, 27, 3526-3532.

280 C. B. Whitehead, M. A. Watzky and R. G. Finke, "Burst Nucleation" vs. Autocatalytic, "Burst" Growth in NearMonodisperse Particle-Formation Reactions, J. Phys. Chem. C, 2020, 124, 24543-24554.

281 P. Coulier, J. Note sur une nouvelle propriete de l'air, J, J. de Pharmacie et de Chimie, Paris, Ser., 1875, 4, 165-173.

282 J. Aitken, On the dust, fog, and clouds, Proc. Roy. Soc., 1882, 11, 14-18.

$283 \mathrm{~J}$. Aitken, On the numbers of dust particles in the atmosphere in certain places in Great Britain and on the continent, with remarks on the relation between the amount of dust and meteorological phenomena, Trans. R. Soc. Edinburgh: Earth Sci., 1889, 35, 1-19.

$284 \mathrm{~J}$. Aitken, On improvements in the apparatus for counting the dust particles in the atmosphere, Proc. Roy. Soc., 1890, 16, 135-172.

285 J. Aitken, On a simple pocket dust-counter, Proc. Roy. Soc., 1892, 18, 39-52.

286 C. T. R. Wilson, Condensation of water vapour in the presence of dust-free air and other gases, Philos. Trans. R. Soc. London, 1897, 189, 265-307.

287 M. Kerker, E. Daby, G. L. Cohen, J. P. Kratohvil and E. Matijevic, Particle size distribution in LaMer sulfur sols, J. Phys. Chem., 1963, 67, 2105-2111.

288 A. J. Petro, Particle size distribution in monodisperse sulfur hydrosols, J. Phys. Chem., 1960, 64, 1508-1511.

289 R. Strey, P. E. Wagner and Y. Viisanen, The Problem of Measuring Homogeneous Nucleation Rates and the Molecular Contents of Nuclei: Progress in the Form of Nucleation Pulse Measurements, J. Phys. Chem., 1994, 98, 7748-7758.

290 K. R. Spurny, Atmospheric Condensation Nuclei P. J. Coulier 1875 and J. Aitken 1880 (Historical Review), Aerosol Sci. Technol., 2000, 32, 243-248.

291 S. A. Seifert, S. Von Essen, K. Jacobitz, R. Crouch and C. P. Lintner, Organic dust toxic syndrome: a review, J. Toxicol., Clin. Toxicol., 2003, 41, 185-193.

292 J. A. Poole and D. J. Romberger, Immunological and inflammatory responses to organic dust in agriculture, Curr. Opin. Allergy Clin. Immunol., 2012, 12, 126-132.

293 S. A. Kulkarni, S. S. Kadam, H. Meekes, A. L. Stankiewicz and J. H. ter Horst, Crystal nucleation kinetics from induction times and metastable zone widths, Cryst. Growth Des., 2013, 13, 2435-2440.

294 L. Granasy, F. Podmaniczky, G. I. Toth, G. Tegze and T. Pusztai, Heterogeneous nucleation of/on nanoparticles: a density functional study using the phase-field crystal model, Chem. Soc. Rev., 2014, 43, 2159-2173.

295 G. Kulkarni, C. Sanders, K. Zhang, X. Liu and C. Zhao, Ice nucleation of bare and sulfuric acid-coated mineral dust particles and implication for cloud properties, J. Geophys. Res.: Atmos., 2014, 119, 9993-10011.

296 S. Özkar and R. G. Finke, Dust Effects on Nucleation Kinetics and Nanoparticle Product Size Distributions: 
Illustrative Case Study of a Prototype $\operatorname{Ir}(0)_{n}$ TransitionMetal Nanoparticle Formation System, Langmuir, 2017, 33, 6550-6562. See also references therein to the early literature of dust in nucleating systems.

297 J. Pelley, Tracing the chemistry of household dust. C\&EN 2017, February 13, pp. 18-21.
298 D. R. Handwerk, P. D. Shipman, S. Özkar and R. G. Finke, Dust Effects on $\operatorname{Ir}(0)_{n}$ Nanoparticle Formation Nucleation and Growth Kinetics and Particle SizeDistributions: Analysis by and Insights from MechanismEnabled Population Balance Modeling, Langmuir, 2020, 36, 1496-1506. 